\title{
Electronic structure with dipole moment and ionicity calculations of the low-lying electronic states of ZnF molecule
}

\begin{tabular}{|r|l|}
\hline Journal: & Canadian Journal of Chemistry \\
\hline Manuscript ID & cjc-2016-0058.R1 \\
\hline Manuscript Type: & Article \\
\hline Complete List of Authors: & $\begin{array}{l}\text { Elmoussaoui, Soumaya; BAU, Physics } \\
\text { El-Kork, Nayla; Khalifa University of Science Technology and Research, } \\
\text { Math and Applied Science } \\
\text { Korek, Mahmoud; Beirut Arab University, }\end{array}$ \\
\hline Keyword: & $\begin{array}{l}\text { Ab initio calculation, electronic structure, spectroscopic constants, potential } \\
\text { energy curves, ionicity, dipole moments, transition dipole moments and } \\
\text { Einstein emission coefficients. }\end{array}$ \\
\hline \multicolumn{2}{|c}{} \\
\hline
\end{tabular}

\section{SCHOLARONE \\ Manuscripts}




\title{
Electronic structure with dipole moment and ionicity calculations of the low-lying electronic states of the $\mathrm{ZnF}$ molecule
}

\author{
Soumaya Elmoussaoui ${ }^{1}$ Nayla El-Kork ${ }^{2}$, and Mahmoud Korek ${ }^{1, *}$ \\ ${ }^{1}$ Department of Physics, Beirut Arab University, P.O.Box 11-5020 Riad El Solh, Beirut 1107 \\ 2809, Lebanon \\ ${ }^{2}$ Khalifa University, P.O. Box 57, Sharjah, United Arab Emirates
}

Shortened title: Theoretical calculations of $\mathrm{ZnF}$ molecule

Key words: $A b$ initio calculation, electronic structure, spectroscopic constants, potential energy curves, ionicity, static and transition dipole moments, Einstein emission coefficients.

Submitted to: Can. J. Chem.

*Author to whom correspondence should be addressed.
Electronic mail: fkorek@yahoo.com,
$\underline{\text { mahmoud.korek@,bau.edu.lb }}$ 


\begin{abstract}
Adiabatic potential energy curves of the 28 low-lying doublet and quartet electronic states in the representation ${ }^{2 s^{+}} \Lambda^{( \pm)}$of the zinc monofluoride molecule are investigated using the complete active space self consistent field (CASSCF) with multi-reference configuration interaction MRCI method including single and double excitations with Davidson correction $(+Q)$. The internuclear distance $R_{e}$, the harmonic frequency $\omega_{e}$, the static and transition dipole moment $\mu$, the rotational constant $\mathrm{B}_{\mathrm{e}}$ and the electronic transition energy with respect to the ground state $T_{e}$ are calculated for the bound states. The transition dipole moment between some doublet states is used to determine the Einstein spontaneous $A_{21}$ and induced emission $B_{21}^{\omega}$ coefficients as well as the spontaneous radiative lifetime $\tau_{\text {spon }}$, emission wavelength $\lambda_{21}$ and oscillator strength $f_{21}$. The ground state ionicity $\mathrm{q}_{\text {ionicity }}$ and equilibrium dissociation energy $\mathrm{D}_{\mathrm{E}, \mathrm{e}}$ are also computed. The comparison between the values of the present work and those available in the literature for several electronic states shows very good agreement. Twenty-three new electronic states have been studied in the present work for the first time.
\end{abstract}




\section{Introduction}

Though transition metal halides are not an easy target for structure determination, their study has received the attention of several theoretical and experimental researchers especially in the field of high temperature chemistry. ${ }^{1}$ Under ordinary conditions most of them are crystals whose molecular forms can only be found in the gaseous phase. With full subshells, the zinc element of group IIB is a transition element of many unique characteristics due to the weak metallic bonding of the $4 s^{2}$ electrons. The complexity of its diatomic halide systems makes theoretical spectroscopic investigations quite interesting; they can be used as guidance for further experimental work and may contribute in the advancement of high energy studies. Among the zinc monohalide species, $\mathrm{ZnF}$ and $\mathrm{ZnCl}$ have been the subject of many experimental and theoretical studies, but few included their excited states. Rochester and Olsson $^{2}$ analyzed an ultraviolet absorption spectrum of $\mathrm{ZnF}$ and reported the ground state vibrational frequency $\omega_{\mathrm{e}}=628 \mathrm{~cm}^{-1}$ and its anharmonicity correction $\omega_{\mathrm{e}} \mathrm{X}_{\mathrm{e}}=3.5 \mathrm{~cm}^{-1}$. For the same state, Moravec et al. ${ }^{3}$ determined $\omega_{\mathrm{e}}=620 \mathrm{~cm}^{-1}$ from photoelectron spectrum of the $\mathrm{ZnF}^{-}$ anion and concluded that the $A^{2} \Pi$ state should lie at more than $21697 \mathrm{~cm}^{-1}$. As a first gasphase spectroscopic investigation, Flory et $a l^{4}$ recorded the pure rotational spectrum of the $\mathrm{ZnF}$ radical using millimeter/submillimeter direct absorption techniques from which the spectroscopic constants of several isotopologues in the ground state are measured. They also estimated the ground state dissociation energy $D_{E}$ and approximated the energy of the $A^{2} \Pi$ state. Theoretically, Bowmaker and Schwerdtfeger ${ }^{5}$ were first to carry out an $a b$-initio calculation using a modified version of Pople's SCF program GAUSSIAN 86. They reported the ground state equilibrium bond length, dissociation energy and force constant of the four diatomic zinc halides. Also for the ground state, the theoretical work of Liao et $a l^{6}$ determined the constants $\mathrm{R}_{\mathrm{e}}$ and $\omega_{\mathrm{e}}$ of the $\mathrm{ZnF}$ free radical using the relativistic corrected local density methods. Boldyrev and Simons ${ }^{7}$ optimized those constants for the two lowest electronic states using analytical gradients and polarized split valence basis sets of 6$331++G(d, f)$ quality using different theoretical methods. Their calculations have shown that the ground state is strongly bound whereas the ${ }^{2} \Pi$ state is a van der Waals complex. Few years later, Belanzoni et al. ${ }^{8}$ reported the bond distances for several molecules including $\mathrm{ZnF}$ molecule by using the density functional approach with the zero order regular approximation (ZORA) method. Based on the assumption that calcium is a pseudo-transition metal, Harrison et $a{ }^{9}{ }^{9}$ suggested that $\mathrm{ZnF}$ and $\mathrm{CaF}$ should have similar bonding properties. They studied the bonding characteristics of the zinc monofluoride system at the $\operatorname{RCCSD}(\mathrm{T})$ level and reported 
$\mathrm{R}_{\mathrm{e}}=1.775 \AA$ and $\omega_{\mathrm{e}}=633 \mathrm{~cm}^{-1}$ for its ground state. Hayashi et al. ${ }^{10}$ employed the multireference configuration interaction method with Davidson correction (MRCI+Q) to plot the adiabatic potential energy curves (PECs) and the dipole moment curves (DMCs) of several low-lying doublet and quartet electronic states. The spectroscopic constants $\mathrm{R}_{\mathrm{e}}, \omega_{\mathrm{e}}, \mathrm{T}_{\mathrm{e}}$ and the absolute value of the dipole moment $\mu$ of 5 low-lying doublet states were reported. Lately, the two lowest excited ${ }^{2} \Pi$ state of this molecule had been diabatized in order to simulate the ${ }^{2} \Pi \leftarrow X^{2} \Sigma^{+}$vibronic spectrum using a wavepacket propagation technique and to study the predissociation of the $\mathrm{A}^{2} \Pi$ state. ${ }^{11}$ In this work, we employed the ab-initio method to plot the potential energy curves (PECs) and the static dipole moment curves (DMCs) of 28 low-lying doublet and quartet electronic states of $\mathrm{ZnF}$ molecule. The spectroscopic constants as the equilibrium internuclear distance $R_{e}$, the harmonic frequency $\omega_{e}$, the vibrational constant $B_{e}$, the transition energy with respect to the ground state minimum $T_{e}$ and the permanent dipole moment $\mu$ are calculated for the bound states. The ground state ionicity $\mathrm{q}_{\text {ionicity }}$ and dissociation energy $\mathrm{D}_{\mathrm{E}}$ are also estimated using the obtained constants. The transition dipole moment between some doublet low-lying states is studied and employed to determine some emission coefficients as the Einstein spontaneous coefficient $A_{21}$, the spontaneous radiative lifetime $\tau_{\text {spon }}$, the wavelength $\lambda_{21}$ and the oscillator strength $f_{21}$.

\section{Computational approach of the ab-initio calculations}

Considering the complete active space self-consistent field (CASSCF) wave functions as reference, the calculations have been performed using the multi-reference configuration interaction (MRCI) method with Davidson correction $(+Q)$, single and double excitations, as applied in the computational chemistry program MOLPRO ${ }^{12}$ and taking the advantage of the graphical user interface GABEDIT. ${ }^{13}$ From the basis set library of the program, the neutral fluorine atom with two core electrons is treated using the ECP2SDF basis set where the 7 valence electrons are described by the associated $(4 s 4 p) /[2 s 2 p]$ basis sets. The neutral zinc atom with 30 electrons is treated via the energy-consistent ECP10MDF basis set as a system of 10 core electrons where the remaining 20 electrons are described by the energy optimized $(12 s 12 p 9 d 3 f 2 g) /[6 s 6 p 4 d 3 f 2 g]$ valence basis sets. ${ }^{14}$ Both effective core potentials consider quasi-relativistic level of theory. The quality of the selected basis sets is checked by comparing our CI calculations for the ground and several excited states of isolated zinc and fluorine atoms to the experimental data in NIST Atomic Spectra Database. ${ }^{15}$ The ionization potential of $\mathrm{Zn}$ and the electron affinity of $\mathrm{F}$ are calculated by comparing the ground state 
atomic and ionic energies. The calculated values, $9.653 \mathrm{eV}$ for $\mathrm{Zn}$ and $3.487 \mathrm{eV}$ for $\mathrm{F}$ are in accordance with those obtained experimentally with the relative differences $2.75 \%$ and $2.54 \%$ respectively. Among the 39 electrons considered for the zinc mono-halide molecule (30 electrons for $\mathrm{Zn}$ and 9 for F), 12 electrons are treated within the core and the wavefunctions of 27 electrons are to be determined. The $3 s, 3 p$ and $3 d$ orbitals of $\mathrm{Zn}$ and the $2 s$ orbital of $\mathrm{F}$ are kept doubly occupied and frozen in the subsequent calculations so that seven valence electrons are explicitly treated. The active space contains at least $6 \sigma\left(\mathrm{Zn}: 4 s, 4 p_{0}, 5 s ; \mathrm{F}: 2 p_{0}\right.$, $3 s, 4 s)$ and $2 \pi\left(\mathrm{Zn}: 4 p_{ \pm 1} ; \mathrm{F}: 2 p_{ \pm 1}\right)$ orbitals in the $\mathrm{C}_{2 \mathrm{v}}$ symmetry group distributed into irreducible representations $a_{1}, b_{1}, b_{2}$, and $a_{2}$ in the following way $6 a_{1}, 2 b_{1}, 2 b_{2}, 0 a_{2}$ noted by $[6,2,2,0]$. In the range of the internuclear distance $\mathrm{R}$ around the equilibrium position of its ground state, the $\mathrm{ZnF}$ molecule is assumed to be mainly ionic. As function of $\mathrm{R}$, the potential energy curves and static dipole moment curves of the doublet and quartet states in the representation of ${ }^{2 \mathrm{~s}^{+1}} \Lambda^{ \pm}$have been calculated for 116 internuclear distances in the range $1.41 \AA \leq \mathrm{R} \leq 4.8 \AA$.

\section{Results and discussion}

The calculations enabled the investigation of 14 low-lying doublet (five ${ }^{2} \Sigma^{+}$, two ${ }^{2} \Sigma^{-}$, two ${ }^{2} \Delta$ and five ${ }^{2} \Pi$ ) and 14 quartet electronic states (five ${ }^{4} \Sigma^{+}$, two ${ }^{4} \Sigma^{-}$, two ${ }^{4} \Delta$ and five ${ }^{4} \Pi$ ). Their PECs and DMCs are plotted as function of the internuclear distance $\mathrm{R}$ as shown in Figures (SM.1 - SM.6). Their numerical data is also provided as supplementary material [SM: B) Numerical data]. Among these states, 19 are found bound. The energy data around the equilibrium position is fitted into a polynomial in terms of the internuclear distance $\mathrm{R}$ in order to calculate the spectroscopic constants $R_{e}, T_{e}, \omega_{e}$ and $B_{e}$. These calculated constants together with the experimental and theoretical values available in literature are given in Table 1 . The ground state, which is the most notably investigated electronic state of $\mathrm{ZnF}$ in literature, is determined to be ${ }^{2} \Sigma^{+}$state similar to the other zinc monohalides. ${ }^{16}$ Its calculated equilibrium bond length $\mathrm{R}_{\mathrm{e}}=1.802 \AA$ is in very good agreement with all the previously reported values, experimentally and theoretically, where the relative difference ranges between $(0.17 \%) \leq \Delta \mathrm{R}_{\mathrm{e}} / \mathrm{R}_{\mathrm{e}} \leq(2.27 \%){ }^{8,11}$ The spectroscopic constants $\omega_{\mathrm{e}}=627.1 \mathrm{~cm}^{-1}, \mathrm{~B}_{\mathrm{e}}=0.3501 \mathrm{~cm}^{-1}$ and $\alpha_{\mathrm{e}}=2.690 \times 10^{-3} \mathrm{~cm}^{-1}$ show reasonable agreement when compared to the most recent experimentally obtained values ${ }^{4}$ where the relative differences are $0.62 \%, 4.97 \%$ and $8.30 \%$ respectively. The calculated ground state vibrational frequency $\omega_{\mathrm{e}}$ is $0.14 \%$ close to the value of $\omega_{\mathrm{e}}=628 \mathrm{~cm}^{-1}$ obtained from UV absorption spectrum analysis. ${ }^{2}$ The anharmonicity 
correction $\omega_{\mathrm{e}} \chi_{\mathrm{e}}=3.8 \mathrm{~cm}^{-1}$ is also compared to the so far determined values, $3.5 \mathrm{~cm}^{-1}, 2 \mathrm{~cm}^{-1}, 4$ $\mathrm{cm}^{-1}, 3.2 \mathrm{~cm}^{-1}$ and $2.8 \mathrm{~cm}^{-1}$, available in literature. ${ }^{2-4,10,11}$

The early experimental work of Rochester and Olsson have assigned a doublet splitting for the ${ }^{2} \Pi \leftarrow^{2} \Sigma^{+}$transition at $36975 \mathrm{~cm}^{-1}$ and $37399 \mathrm{~cm}^{-1}$. Consistently, the photoelectron spectrum of the $\mathrm{ZnF}^{-}$anion ${ }^{3}$ showed that no additional spectrum in the electronic structure of $\mathrm{ZnF}$ is obtained below $21697 \mathrm{~cm}^{-1}$ and that the first ${ }^{2} \Pi$ state should lie higher. However while analyzing the second-order spin-orbit coupling for $\mathrm{ZnF}$ and considering the $\mathrm{A}^{2} \Pi$ state as the main perturber to the ground state, the energy of the excited state is approximated to be 20400 $\mathrm{cm}^{-1}{ }^{4}$ The theoretical calculations of the lowest states of $\mathrm{ZnF}$ at the QCISD level ${ }^{7}$ reported that the lowest ${ }^{2} \Pi$ state is a van der Waals complex whose minimum at $4.509 \AA$ and $\mathrm{T}_{\mathrm{e}}=22044 \mathrm{~cm}^{-1}$ whereas $\operatorname{RCCSD}(\mathrm{T})$ calculations ${ }^{9}$ concluded that this state is an isolated one characterized by $\mathrm{T}_{\mathrm{e}}=37344 \mathrm{~cm}^{-1}$ at $1.757 \AA$ and correlated with the second asymptote. Motivated by the wide range of the reported transition energy values $T_{e}$ for the lowest ${ }^{2} \Pi$ state of $\mathrm{ZnF}$, a quasi-diabatic study at the MRCI level of theory has been carried out ${ }^{11}$ at the region of $36300 \mathrm{~cm}^{-1}$ above the ground state in order to optimize a previous adiabatic study in which the electronic structure and the spectroscopic constants of few low-lying states were calculated within MRCI+Q method. ${ }^{10}$ The procedure lead to two ${ }^{2} \Pi$ states; a repulsive one reaching the first dissociation limit and a bound one associated with the second limit. In this work, though the first ${ }^{2} \Pi$ state that correlates with the first asymptote describes a very shallow PEC with minimum energy of $T_{e}=19611 \mathrm{~cm}^{-1}$ at $3.831 \AA$ indicating a nearly dissociative nature; yet this state supports very few vibrational levels $(v=0,1,2)$. However, the calculations predict that the transitions to this state are almost negligible (see the discussion of the transition dipole moment below). The shoulder of the first ${ }^{2} \Pi$ state at the avoided crossing region with a higher ${ }^{2} \Pi$ state at $1.83 \AA$ yields a vertical excitation of $36247 \mathrm{~cm}^{-1}$ at the equilibrium geometry of the $X^{2} \Sigma^{+}$state. A transition energy of $T_{e}=37515 \mathrm{~cm}^{-1}$ at $R_{e}=1.884 \AA$ of the $(2)^{2} \Pi$ state is in excellent agreement with the values existing in literature as the relative difference ranges between $(0.31 \%) \leq \Delta \mathrm{T}_{\mathrm{e}} / \mathrm{T}_{\mathrm{e}} \leq(4.61 \%)$. ${ }^{9,10}$

In the absence of experimental data apart from the states discussed above, the spectroscopic constants of all other states, given in Table 1, are predictions that may guide future experiments concerning the low-lying excited states of $\mathrm{ZnF}$ molecule. The interaction of the $(2)^{2} \Sigma^{+}$state with the ground state at the region near $2.523 \AA$ results a well whose minimum corresponds to a transition energy of $T_{\mathrm{e}}=31091 \mathrm{~cm}^{-1}$ and vibrational frequency $\omega_{\mathrm{e}}=326.2 \mathrm{~cm}^{-1}$. 
Such transition has received significant attention among the other zinc monohalides ever since the demonstration of the $\mathrm{ZnI}$ lasing. ${ }^{17}$ Our spectroscopic constants of the excited ${ }^{2} \Sigma^{+}$states are compared to those computed theoretically. ${ }^{10,11}$ Although diabatic approximation has yielded better vibrational frequency of the $(2)^{2} \Pi$ state, reasonable agreements are shown for the other constants. The PEC of each of the $(3)^{2} \Sigma^{+},(4)^{2} \Sigma^{+}$and $(5)^{2} \Sigma^{+}$states interacts in turn with a lower and a higher ${ }^{2} \Sigma^{+}$state showing successive positions of avoided crossing as listed in Table 2. Such behavior leads to difficulty in accurate descriptions near the vicinity of these positions. Several crossing points take place between the PECs of the different doublet states as they exist at close energy levels. Table 2 shows these crossing points as well.

Relying on the good accordance obtained so far with literature for the doublet states of $\mathrm{ZnF}$ molecule, we were motivated to advance towards its quartet states. For the first time, the PECs of 8 quartet states are plotted as shown in Figure (SM.2). The spectroscopic constants of 7 bound quartet states are given in Table 1 . The PECs of the $(1)^{4} \Sigma^{+},(1)^{4} \Delta$ and $(1)^{4} \Sigma^{-}$states are found in this work to be bound with shallow minima at $2.762 \AA, 2.956 \AA$ and $3.142 \AA$, respectively. These shallow electronic states, however, support few vibrational levels whose eigenvalues $\mathrm{E}_{v}$, the rotational constants $\mathrm{B}_{v}$, the centrifugal distortion constants $\mathrm{D}_{v}$, the abscissas of the turning points $\mathrm{R}_{\min }$ and $\mathrm{R}_{\max }$ and some calculated wavelengths of possible vibronic transitions are given in Table (SM.3) in the supplementary material (SM: C).

With two ways to specify the dissociation energy, the zero point energy $D_{E, v=0}$ and the equilibrium dissociation energy $D_{E, e}$ can be estimated in terms of the molecular spectroscopic constants. ${ }^{18}$ Our value of $D_{E, e}=73.9 \mathrm{kcal} / \mathrm{mol}$ has yielded $D_{E, v=0}=73.0 \mathrm{kcal} / \mathrm{mol}$. An excellent agreement is shown when the calculated equilibrium dissociation energy is compared to the values available in literature. The relative differences are $1.39 \%, 0.14 \%, 4.43 \%$ and $7.99 \%$ with respect to the values of $72 \mathrm{kcal} / \mathrm{mol}, 72.9 \mathrm{kcal} / \mathrm{mol}, 69.9 \mathrm{kcal} / \mathrm{mol}$ and $67.6 \mathrm{kcal} / \mathrm{mol}$ respectively. ${ }^{4,9,10,7}$ This agreement deteriorates upon comparison with the values of 50.2 $\mathrm{kcal} / \mathrm{mol}$ and $46.8 \mathrm{kcal} / \mathrm{mol}$ reported from MP2 and CISDSD methods. ${ }^{5}$

Since the strength of the vibrational excitations depends on the variation of the dipole moment as function of geometry, ${ }^{19}$ the static dipole moment of the considered states is calculated and plotted as function of the internuclear distance R (Figures SM.3-6). With the zinc atom at the origin, the sign convention is defined such as the negative value corresponds to the $\mathrm{Zn}^{\delta+} \mathrm{F}^{\delta-}$ 
polarity. The curves will be mainly used here to indicate the bond nature, the polarity and interaction of the states. The DMC of the ground state presents negatively increased values with the largest magnitude $|\mu|=1.61$ a.u. near $\mathrm{R}=2.31 \AA$. The electric dipole moment curve shows a nearly linear trend close to the equilibrium position $R_{e}$ for this ground state. One can understand the behavior of a bond from such curve by using the Paccinioni et al. ${ }^{20}$ method: For two charges $+q$ and $-\mathrm{q}$ separated by a distance $\mathrm{R}$, the dipole moment is a straight line if the compound formed by the two charges is ionic, and curved if the compound has a dominating covalent character. This idea was quantified as the following: If $\mu=-q \times R$, then $\partial \mu \partial R=-q$, the larger value of $\mathrm{q}$, the more ionic charge (except in some case of polarization effects) and $\partial^{2} \mu / \partial R^{2}$ is closer to zero as the ionicity increases. In our case, $\partial \mu / \partial R=-1.48$ a.u $/ \AA$ and $\partial^{2} \mu / \partial R^{2}=1.1$; the negative linear slope of value -1.48 confirms the presence of the ionic character of $\mathrm{ZnF}$ bond of the ground state, and the value 1.1 of the curvature indicates also a covalent nature of the same bond. This mixed nature has been confirmed experimentally by Flory et al., ${ }^{4}$ who through the evaluation of hyperfine parameters has confirmed that $\mathrm{ZnF}$ is an ionic species, but with some covalent character. Another parameter that helps to quantify the nature of a molecular bond is the electric dipole moment charge, or the ionicity of a molecule defined as $q_{\text {ionicity }}=\mu / e R_{e} \cdot{ }^{21}$ This quantity describes the fraction of ionic character of a molecular bond. ${ }^{22,23} \mathrm{We}$ calculate the ionicity of the $\mathrm{ZnF}$ molecule by applying the formula given above for the ground state at its equilibrium distance $R_{e}$. The dipole moment $\mu$ and internuclear distance $R_{e}$ are defined from the potential energy and dipole moment curves, Figures (SM.1, SM.3), at the equilibrium position. The comparison of the calculated value of the ionicity $q_{\text {ionicity }}=\frac{\mu}{e R_{e}}=0.32$ for the ground state with other data available in literature shows an overall good agreement (Table 3) which confirm the mixed nature (ionic/covalent) of the bond. At large internuclear distances where the molecule dissociates into its natural fragments, the dipole moment is expected to approach zero smoothly which is the case for the investigated states. However, the significant increase in the absolute dipole moment value of $(4)^{2} \Sigma^{+}$state at distances between $3.4 \AA$ and $6.4 \AA$ points out a polarized state at this region where the fluorine atom strongly attract the bonding electrons. But upon extending the calculations to larger internuclear distances, the dipole moment possesses quasi-zero values at $\mathrm{R}>8 \AA$. The abrupt gradient change of the DMCs can be related to the occurrence of an avoided crossing between the PECs of two states of the same symmetry. At this region, the wavefunctions of these states will mix with each other to give 
two adiabatic solutions such that the corresponding PECs do not cross. The DMCs mirror this region as a sharp change in their slopes indicating a reversed polarity of the atoms as for $(1)^{2} \Pi-(2)^{2} \Pi$ at $1.83 \AA$ and $(2)^{2} \Sigma^{+}-(3)^{2} \Sigma^{+}$at $1.68 \AA$. The behavior of the dipole moment of the $(5)^{2} \Sigma^{+}$state between $1.86 \AA$ and $1.77 \AA$ is referred to its non-crossing interaction with a higher and a lower ${ }^{2} \Sigma^{+}$states successively. The validity of excited states calculations may be confirmed by noticing the agreement between regions of anti-crossing of two PECs and the crossing of their corresponding DMCs. Those positions are marked by dashed ovals on the DMCs.

Moreover, calculations of the PECs and DMCs show another importance for heteronuclear diatomic molecules as they allow noticing the non-zero permanent dipole moment at the equilibrium geometry (given in Table 1) which indicates the unequal distribution of electrons between the two nuclei. As a result, there can be a small excess of negative charge near one of the atoms and thus the molecule in this state is said to be a polar molecule. Polar molecules can be manipulated by external electric fields, allowing for the control of elementary chemical reactions at very low temperatures. There are several applications of such molecules in the physics of ultra-cold molecular gases. For example, the long-range electric dipole-dipole interaction between two polar molecules can significantly modify the many-body dynamics of trapped ultra-cold molecular Bose Einstein Condensates. For such application and others, it is known that the knowledge of the permanent or transition dipole moments is essential. ${ }^{24}$ Therefore, the transition dipole moment curves (TDMs) between some doublet states are investigated and plotted in Figures (SM.7, SM.8). These curves are considered important as they can be very useful in designing the photoassociation experiments for a molecule ${ }^{25}$ and helpful to predict transitions. Using the transition dipole moment value between two states $\mu_{21}$ at its equilibrium position of the upper state, we calculated the Einstein coefficient of spontaneous emission $A_{21}$ from which the spontaneous radiative lifetime $\tau_{\text {spon }}$, the Einstein coefficient of induced emission $B_{21}^{\omega}$, the corresponding emission cross section $\sigma_{0}$, the classical radiative decay rate of the single-electron oscillator at the emission frequency $\gamma_{c l}$ and the emission oscillator strength $f_{21}$ are determined by applying the following formulas: ${ }^{26}$

$$
\begin{gathered}
\omega_{21}=2 \pi v_{21} \\
A_{21}=\frac{2 \omega_{21}^{3} \mu_{21}^{2}}{3 \varepsilon_{0} h c^{3}}
\end{gathered}
$$




$$
\begin{gathered}
\tau_{\text {spon }}=\frac{1}{A_{21}} \\
B_{21}^{\omega}=\frac{\pi^{2} c^{3} A_{21}}{\hbar \omega_{21}^{3}} \\
\sigma_{0}=\frac{g_{2} \lambda_{21}^{2} A_{21}}{4 g_{1}} \\
\gamma_{c l}=\frac{e^{2} \omega_{21}^{2}}{6 \pi \varepsilon_{0} m_{e} c^{3}} \\
f_{21}=\frac{-A_{21}}{3 \gamma_{c l}}
\end{gathered}
$$

where $v_{21}$ is the transition frequency between the two states, $h$ is Plank's constant, $\varepsilon_{0}$ is the vacuum permittivity, $c$ is the velocity of light and $g_{1}$ and $g_{2}$ are the degeneracy factor of the lower and upper state, respectively, as indicated from the multiplicity and by neglecting the rotational and vibrational factors such that: ${ }^{27}$

$$
g_{L}= \begin{cases}(2 S+1) & \text { if } \Lambda=0 \\ 2(2 S+1) & \text { if } \Lambda \neq 0\end{cases}
$$

The calculated constants are given in Table 4. It can be deduced that the intensity of some transitions is neglected as for the transitions from the $(1)^{2} \Delta$ and $(1)^{2} \Sigma^{-}$states where $\left|f_{21}\right|<$ $10^{-4}$ is very small when compared to those of the experimentally verified transitions, i.e., $\mathrm{X}^{2} \Sigma^{+}-(2)^{2} \Sigma^{+}$and $\mathrm{X}^{2} \Sigma^{+}-(2)^{2} \Pi$. It is also clear that most of these transitions take place in the ultraviolet region. As for the TDMCs, the sudden changes and jumps, that appear at different internuclear distances, can be explained either by the existence of avoided crossings between the potential energy curves at these particular distances $\left((1)^{2} \Pi^{+} /(2)^{2} \Pi^{+}\right.$, Figures (SM.1, SM.4)) or referred to a strong interaction of the covalent state with the ion-pair state (responsible for the electron transfer) and to the change of sign of the electronic wavefunctions around these internuclear distances. The curves as well as the corresponding numerical data of the TDMs between different electronic states in terms of the internuclear distance $\mathrm{R}$ are given as supplementary material in [SM: A), B)].

\section{Conclusion}


Using the ab-initio method as implemented in the MRCI+Q calculations, we have investigated the adiabatic potential energy curves and the dipole moment curves for 14 doublet and 14 quartet low-lying electronic states of $\mathrm{ZnF}$ molecule considering 7 valence electrons. The plots of these curves have been represented and the spectroscopic constants $R_{e}$, $\omega_{\mathrm{e}}, \mathrm{T}_{\mathrm{e}}$, and $\mathrm{B}_{\mathrm{e}}$ are determined for 19 bound states. The ionicity $q_{\text {ionicity }}$ and the equilibrium dissociation energy $\mathrm{D}_{\mathrm{E}, \mathrm{e}}$ of the $\mathrm{X}^{2} \Sigma^{+}$state are also computed. The comparisons with the available low-lying theoretically plotted curves provide almost perfect agreement. The bond of the ground state is confirmed to be of mixed nature (ionic/covalent). The calculated spectroscopic constants are in very good accordance with other theoretical and experimental results. The calculated transition dipole moments were used to determine the Einstein spontaneous emission coefficients $A_{21}$ from which other emission constants are calculated to shed light on few possible low-lying transitions.

\section{Acknowledgements}

The authors would like to acknowledge the use of the Ankabut High Power Computer Facility Cluster and the Khalifa University High Power Computer shared facility, Abu-Dhabi UAE. Also, the authors would like to thank the Lebanese National Council for Scientific Research for the financial support of the present work by the grant program for scientific research 20132015. 


\section{References}

${ }^{1}$ Carlson, K. D.; Claydon, C. R.. Adv. High Temp. Chem. 1967, 1, 43. doi:10.1016/S00652741(13)70022-8

${ }^{2}$ Rochester, G. D., Olsson, E. Z. Phys. 1939, 114, 495. doi: 10.1007/BF01329529

${ }^{3}$ Moravec, V. D.; Klopcic, S. A.; Chatterjee, B.; Jarrold, C. C. Chem. Phys. Lett. 2001, 341, 313. doi:10.1016/S0009-2614(01)00465-1

${ }^{4}$ Flory, M. A.; McLamarrah, S. K.; Ziurys, L. M. J. Chem. Phys. 2006, 125, 194304. doi: $\underline{10.1063 / 1.2355495}$

${ }^{5}$ Bowmaker, G. A.; Schwerdtfeger, P. J. Mol. Struct.: THEOCHEM. 1990, 205, 295. doi: $\underline{10.1016 / 0166-1280(90) 85129-\mathrm{B}}$

${ }^{6}$ Liao, M.; Zhang, Q.; Schwarz, W. H. E. Inorg. Chem. 1995, 34, 5597. doi: $\underline{10.1021 / \mathrm{ic} 00126 \mathrm{a} 033}$

${ }^{7}$ Boldyrev, A. I.; Simons, J. Mol. Phys. 1997, 92, 365-379. doi:10.1080/002689797170103

${ }^{8}$ Belanzoni, P.; Lenthe, E. van; Baerends, E. J. J. Chem. Phys. 2001, 114, 4421. doi: $\underline{10.1063 / 1.1345509}$

${ }^{9}$ Harrison, J. F.; Field, R. W.; Jarrold, C. C. ACS Sym. Ser. 2002, 828, 238. doi: 10.1021/bk2002-0828.ch011

${ }^{10}$ Hayashi, S.; Léonard, C.; Chambaud, G. J. Chem. Phys. 2008, 129, 044313. doi: $\underline{10.1063 / 1.2960585}$

${ }^{11}$ Léonard, C.; Le Quéré, F. J. Chem. Phys. 2012, 137, 164318. doi:10.1063/1.4762444

${ }^{12}$ Werner, H. J.; Knowles, P. J.; Lindh, R.; Manby, F. R.; Schütz, M.; Celani, P.; Korona, T.; Rauhut, G.; Amos, R. D.; Bernhardsson, A.; Berning, A.; Cooper, D. L.; Deegan, M. J. O.; Dobbyn, A. J.; Eckert, F.; Hampel, C.; Hetzer, G.; Lloyd, A. W.; McNicholas, S. J.; Meyer, W.; Mura, M. E.; Nicklass, A.; Palmieri, P.; Pitzer, R.; Schumann, U.; Stoll, H.; Stone, A. J.; Tarroni, R.; Thorsteinsson, T. 2008. Version 2012.1; http://www.molpro.net/info/users

${ }^{13}$ Allouche, A. R. Gabedit: J. Comp. Chem. 2011, 32, 174. doi: 10.1002/jcc.21600

${ }^{14}$ Figgen, D.; Rauhut, G.; Dolg, M.; Stoll, H. Chem. Phys. 2005, 311, 227. doi:10.1016/j.chemphys.2004.10.005

${ }^{15}$ NIST Atomic Spectra Database. Levels Form. http://www.nist.gov/pml/data/asd.cfm (accessed April 15, 2016).

${ }^{16}$ Huber, K. P.; Herzberg, G. Molecular Spectra and Molecular Structure: Constants of Diatomic Molecules. Van Nostrand Reinhold Company; New York, 1979; pp. 676-677. ISBN: 978-1-4757-0963-6 (Print) 978-1-4757-0961-2

${ }^{17}$ McCown, A. W.; Eden, J. G. Appl. Phys. Lett. 1981, 39, 371. doi:10.1063/1.92741 
${ }^{18}$ Herzberg, G. Molecular Spectra and Molecular Structure: Spectra of Diatomic Molecules. 2nd ed.; D. Van Nostrand Company (Canada), Inc.; Toronto, 1950; pp 100. ISBN-13: 9780894642685 / ISBN-10: 0894642685

${ }^{19}$ Tennyson, J. J. Mol. Spectrosc. 2014, 298, 1. doi:10.1016/j.jms.2014.01.012

${ }^{20}$ Gianfranco, P.; Illas, F. Chem. phys. 1995, 199, 155. doi: 10.1016/0301-0104(95)00202-Y

${ }^{21}$ Stradella, O. G.; Eduardo, E. A.; Fernandez, F. M. Inorganic. Chem. 1951, 24, 3631. doi: $\underline{10.1021 / \mathrm{ic} 00216 \mathrm{a} 031}$

${ }^{22}$ Rittner, E. S. J. Chem. Phys. 1951, 19, 1030. doi: 10.1063/1.1748448

${ }^{23}$ Noorizadeh, S.; Shakerzadeh, E. J. Phys. Chem. A 2008, 112, 3486. doi: 10.1021/jp709877h

${ }^{24}$ Kotochigova, S.; Julienne, P. S.; Tiesinga, E. Phys. Rev. A 2003, 68, 022501. doi: 10.1103/PhysRevA.68.022501

${ }^{25}$ de Lima, E. F.; Ho, T. S.; Rabitz, H. Chem. Phys. Lett. 2011, 501, 267. doi:10.1016/j.cplett.2010.12.018

${ }^{26}$ Hilborn, R. C. Am. J. Phys. 1982, 50, 982. Revised version, 2002. doi: 10.1119/1.12937

${ }^{27}$ Loh, W. H. T. Re-entry and Planetary Entry Physics and Technology. Part 1: Dynamics, Physics, Radiation, Heat Transfer and Ablation. Springer-Verlag New York Inc. 1968; pp. 235. ISBN 978-3-642-48927-3 


\section{Figure captions:}

Figure (SM.1): Potential energy curves of the doublet electronic states of the $\mathrm{ZnF}$ molecule.

Figure (SM.2): Potential energy curves of the quartet electronic states of $\mathrm{ZnF}$.

Figure (SM.3): Dipole moment curves of the ${ }^{2} \Sigma^{+}$electronic states of the $\mathrm{ZnF}$ molecule.

Figure (SM.4): Dipole moment curves of the ${ }^{2} \Sigma^{-},{ }^{2} \Pi$ and ${ }^{2} \Delta$ electronic states of the $\mathrm{ZnF}$ molecule.

Figure (SM.5): Dipole moment curves of the ${ }^{4} \Sigma^{+}$and ${ }^{4} \Sigma^{-}$electronic states of the $\mathrm{ZnF}$ molecule.

Figure (SM.6): Dipole moment curves of the ${ }^{4} \Pi$ and ${ }^{4} \Delta$ electronic states of the $\mathrm{ZnF}$ molecule. Figure (SM.7): Transition dipole moment between the ground and $(2)^{2} \Sigma^{+},(4)^{2} \Sigma^{+},(1)^{2} \Pi$, and $(2)^{2} \Pi$ electronic states of the $\mathrm{ZnF}$ molecule.

Figures (SM.8-a) and (SM.8-b): Transition dipole moments between some doublet electronic states of the $\mathrm{ZnF}$ molecule. 
Table 1: Calculated spectroscopic constants for the bound doublet and quartet electronic states of the $\mathrm{ZnF}$ molecule.

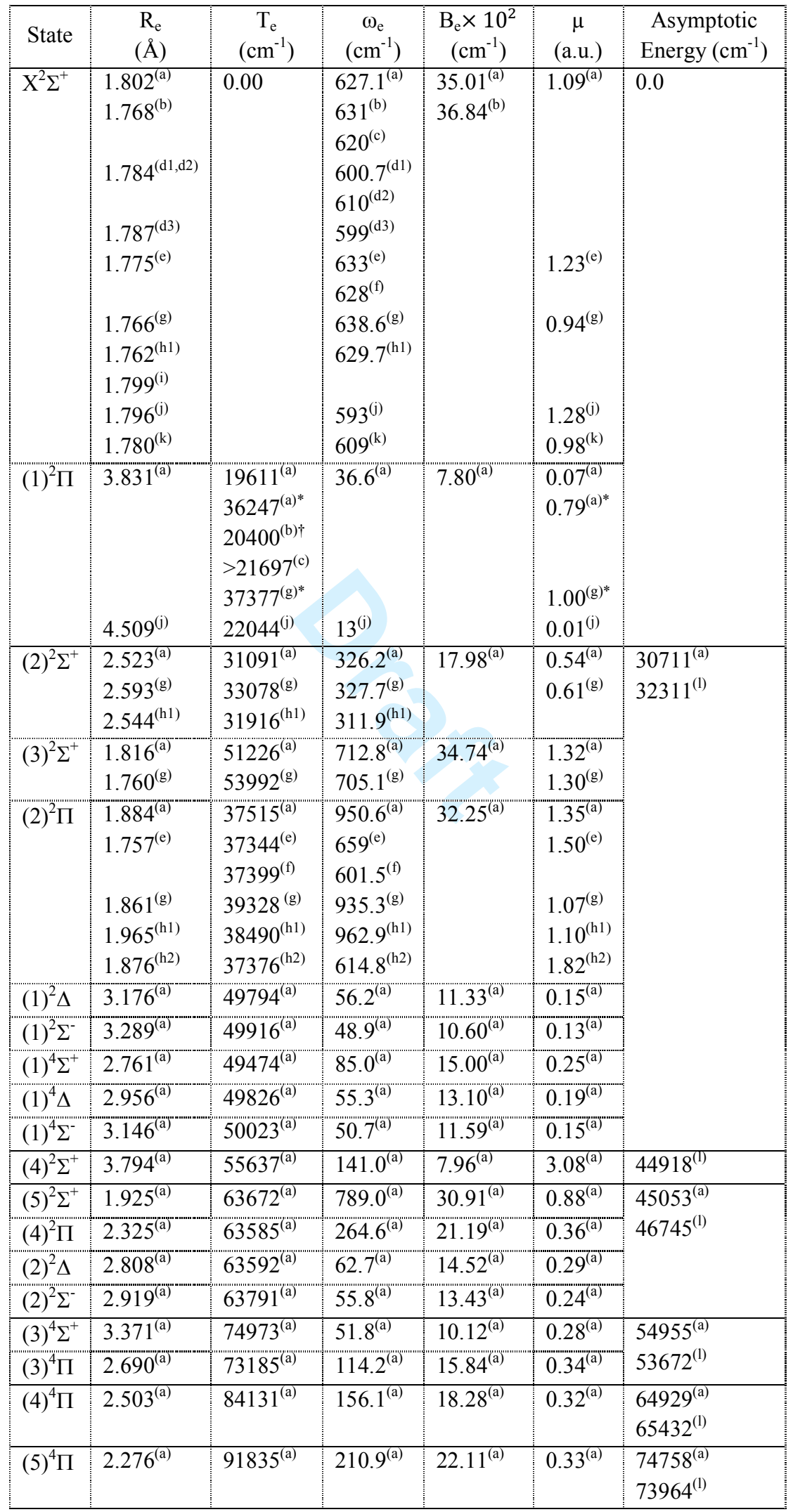




\author{
(a)This work $\left\{{ }^{\text {Theo: }} \mathrm{MRCI}+\mathrm{Q}\right\}$ \\ (b)Flory et al. ${ }^{4}\left\{{ }^{\text {Exp: }}\right.$ Direct absorption techniques\}, $\dagger$ By calculation. \\ (c)Moravec et al. ${ }^{3}$ \{xp: Photoelectron spectra\} \\ (d)Bowmaker and Schwerdtfeger ${ }^{5}\left\{{ }^{\text {Theo: } \mathrm{d} 1}\right.$ MP $2,{ }^{\mathrm{d} 2}$ CISD, ${ }^{\mathrm{d} 3}$ CISDSC $\}$ \\ (e)Harrison et al. ${ }^{9}\left\{{ }^{\text {Theo: }} \operatorname{RCCSD}(\mathrm{T})\right\}$ \\ (f)Rochester and Olsson ${ }^{2}$ \{ ${ }^{\text {Exp: }} \mathrm{UV}$ absorption spectrum\} \\ (g)Hayashiet al. ${ }^{10}{ }^{\text {Theo: }}$ MRCI+Q\} \\ (h)Léonard and Le Quéré ${ }^{11}$ \{ ${ }^{\text {Theo: h1 }}$ adiabatic MRCI,${ }^{\text {h2 }}$ diabatic MRCI\} \\ (i)Belanzoniet al. ${ }^{8}\left\{{ }^{\text {Theo: }}\right.$ DFT-ZORA $\}$ \\ (j)Boldyrev and Simons ${ }^{7}\left\{{ }^{\text {Theo: }}\right.$ QCISD $\}$ \\ (k)Liao et al. ${ }^{6}$ \{heo: $\left.\mathrm{LDF}\right\}$ \\ (1)NIST Atomic Spectra Database ${ }^{15}$ \\ ${ }^{*}$ absolute energy/dipole moment calculated at the minimum of the ground state $\mathrm{X}^{2} \Sigma^{+}$.
}


Table 2: Positions of the crossings $\mathrm{R}_{\mathrm{C}}$ and avoided crossings $\mathrm{R}_{\mathrm{AC}}$ between electronic statesof $\mathrm{ZnF}$ molecule.

\begin{tabular}{|c|c|c|c|}
\hline$\left(\mathrm{n}_{1}\right)$ state $1 /\left(\mathrm{n}_{2}\right)$ state 2 & $R_{C}(\AA)$ & $R_{A C}(\AA)$ & $\Delta \mathrm{E}\left(\mathrm{cm}^{-1}\right)$ \\
\hline$(2)^{2} \Sigma^{+} /(2)^{2} \Pi$ & $\begin{array}{l}1.59 \\
1.98\end{array}$ & & \\
\hline$(3)^{2} \Sigma^{+} /(1)^{2} \Delta$ & 2.16 & & \\
\hline$(3)^{2} \Sigma^{+} /(1)^{2} \Sigma^{-}$ & 2.19 & & \\
\hline$(4)^{2} \Sigma^{+} /(1)^{2} \Delta$ & 1.86 & & \\
\hline$(4)^{2} \Sigma^{+} /(1)^{2} \Sigma^{-}$ & 1.89 & & \\
\hline$(4)^{2} \Sigma^{+} /(3)^{2} \Pi$ & 2.43 & & \\
\hline$(5)^{2} \Sigma^{+} /(3)^{2} \Pi$ & 2.06 & & \\
\hline$(5)^{2} \Sigma^{+} /(4)^{2} \Pi$ & $\begin{array}{l}2.08 \\
2.67\end{array}$ & & \\
\hline$(5)^{2} \Sigma^{+} /(1)^{2} \Delta$ & 1.71 & & \\
\hline$(5)^{2} \Sigma^{+} /(1)^{2} \Sigma^{-}$ & 1.73 & & \\
\hline$(5)^{2} \Sigma^{+} /(2)^{2} \Delta$ & 2.13 & & \\
\hline$(5)^{2} \Sigma^{+} /(2)^{2} \Sigma^{-}$ & 2.19 & & \\
\hline$(4)^{4} \Sigma^{+} /(5)^{4} \Pi$ & 1.98 & & \\
\hline$(2)^{2} \Sigma^{+} /(3)^{2} \Sigma^{+}$ & & 1.68 & 2499 \\
\hline$(3)^{2} \Sigma^{+} /(4)^{2} \Sigma^{+}$ & & $\begin{array}{l}1.47 \\
2.10\end{array}$ & $\begin{array}{c}681 \\
4284\end{array}$ \\
\hline$(4)^{2} \Sigma^{+} /(5)^{2} \Sigma^{+}$ & & 1.86 & 5103 \\
\hline$(1)^{2} \Pi /(2)^{2} \Pi$ & & 1.83 & 2398 \\
\hline$(4)^{4} \Pi /(5)^{4} \Pi$ & sine & 1.56 & 235 \\
\hline
\end{tabular}


Table 3: Calculation of ionicity for the ground $\mathrm{X}^{2} \Sigma^{+}$state of $\mathrm{ZnF}$ molecule.

\begin{tabular}{c|c|c}
\hline$\mu(\mathrm{a} . \mathrm{u})$ & $\mathrm{R}_{\mathrm{e}}(\AA)$ & $\mu / \mathrm{e}_{\mathrm{e}}$ \\
\hline 1.092 & 1.802 & 3.211 \\
\hline $1.23^{(\mathrm{a})}$ & 1.787 & 3.643 \\
\hline $0.98^{(\mathrm{b})}$ & 1.780 & 2.914 \\
\hline $1.28^{(\mathrm{c})}$ & 1.796 & 3.772 \\
\hline
\end{tabular}

First entry is for the present work

(a)Bowmaker and Schwerdtfeger ${ }^{5}$ \{heo: CISDSC $\}$

(b)Liao et al. ${ }^{6}\left\{{ }^{\text {Theo: }} \mathrm{LDF}\right\}$

(c)Boldyrev and Simons ${ }^{7}$ \{heo: $\left.{ }^{\text {QCISD }}\right\}$ 
Table 4: The transition dipole moment values of the upper state at its equilibrium position $|\mu|$, the emission angular frequency $\omega_{21}$, the Einstein coefficients of spontaneous and induced emissions $\left(A_{21}\right.$ and $\left.B_{21}^{\omega}\right)$, the spontaneous radiative lifetime $\tau_{\text {spon }}$, the emission cross section $\sigma_{0}$, the classical radiative decay rate of the single-electron oscillator $\gamma_{c l}$ and the emission oscillator strength $f_{21}$ of some transitions among the doublet states of $\mathrm{ZnF}$.

\begin{tabular}{|c|c|c|c|c|c|c|c|c|c|c|}
\hline Transition & $\begin{array}{l}\left|\mu_{21}\right| \\
\text { (a.u.) }\end{array}$ & $\begin{array}{c}\left|\mu_{21}\right| \times 10^{30} \\
\text { (C.m) }\end{array}$ & $\begin{array}{c}\omega_{21} \times 10^{-15} \\
\left(\mathrm{rad} . \mathrm{s}^{-1}\right)\end{array}$ & $\begin{array}{c}A_{21} \times 10^{-7} \\
\left(\mathrm{~s}^{-1}\right)\end{array}$ & $\begin{array}{c}\tau_{\text {spon }} \\
\text { (ns) }\end{array}$ & $\begin{array}{c}B_{21}^{\omega} \times 10^{-20} \\
\left(\mathrm{~m}^{3} \cdot \operatorname{rad} . J^{-1} \cdot \mathrm{s}^{-2}\right)\end{array}$ & $\begin{array}{c}\sigma_{0} \times 10^{6} \\
\left(\mathrm{~m}^{2} \cdot \mathrm{rad} \cdot \mathrm{s}^{-1}\right)\end{array}$ & $\lambda_{21}(\mathrm{~nm})$ & $\begin{array}{c}\gamma_{c l} \times 10^{-7} \\
\left(\mathrm{~s}^{-1}\right)\end{array}$ & $\left|f_{21}\right|$ \\
\hline $\mathrm{X}^{2} \Sigma^{+}-(1)^{2} \Pi$ & 0.018 & 0.15 & 3.694 & $<10^{-3}$ & $>10^{5}$ & $<10^{-3}$ & 0.001 & 510.0 & 8.55 & $<10^{-4}$ \\
\hline$X^{2} \Sigma^{+}-(2)^{2} \Sigma^{+}$ & 1.223 & 10.37 & 5.856 & 9.11 & 10.98 & 11.43 & 2.355 & 321.7 & 21.49 & 0.141 \\
\hline$(2)^{2} \Pi-(1)^{2} \Delta$ & 0.003 & 0.03 & 2.313 & $<10^{-3}$ & $>10^{5}$ & $<10^{-3}$ & $<10^{-3}$ & 814.5 & 3.35 & $<10^{-4}$ \\
\hline$(1)^{2} \Pi-(1)^{2} \Sigma^{-}$ & 0.001 & 0.01 & 5.708 & $<10^{-3}$ & $>10^{5}$ & $<10^{-3}$ & $<10^{-3}$ & 330.0 & 20.42 & $<10^{-4}$ \\
\hline$(2)^{2} \Pi-(1)^{2} \Sigma^{-}$ & 0.021 & 0.18 & 2.336 & $<10^{-3}$ & $>10^{5}$ & $<10^{-3}$ & $<10^{-3}$ & 806.4 & 3.42 & $<10^{-4}$ \\
\hline$(1)^{2} \Pi-(2)^{2} \Pi$ & 1.044 & 8.85 & 3.372 & 1.27 & 78.92 & 8.33 & 0.988 & 558.6 & 7.13 & 0.059 \\
\hline$X^{2} \Sigma^{+}-(2)^{2} \Pi$ & 1.067 & 9.05 & 7.066 & 12.18 & 8.21 & 8.70 & 4.327 & 266.6 & 31.29 & 0.130 \\
\hline$X^{2} \Sigma^{+}-(3)^{2} \Sigma^{+}$ & 0.767 & 6.50 & 9.648 & 16.02 & 6.24 & 4.50 & 1.526 & 195.2 & 58.34 & 0.092 \\
\hline$X^{2} \Sigma^{+}-(4)^{2} \Sigma^{+}$ & 0.415 & 3.52 & 10.479 & 6.01 & 16.64 & 1.32 & 0.485 & 179.7 & 68.82 & 0.029 \\
\hline$(2)^{2} \Sigma^{+}-(3)^{2} \Sigma^{+}$ & 0.146 & 1.24 & 3.792 & 0.04 & 2837.06 & 0.16 & 0.022 & 496.7 & 9.01 & 0.001 \\
\hline
\end{tabular}




\section{Supplementary Material:}

\section{A. Curves:}

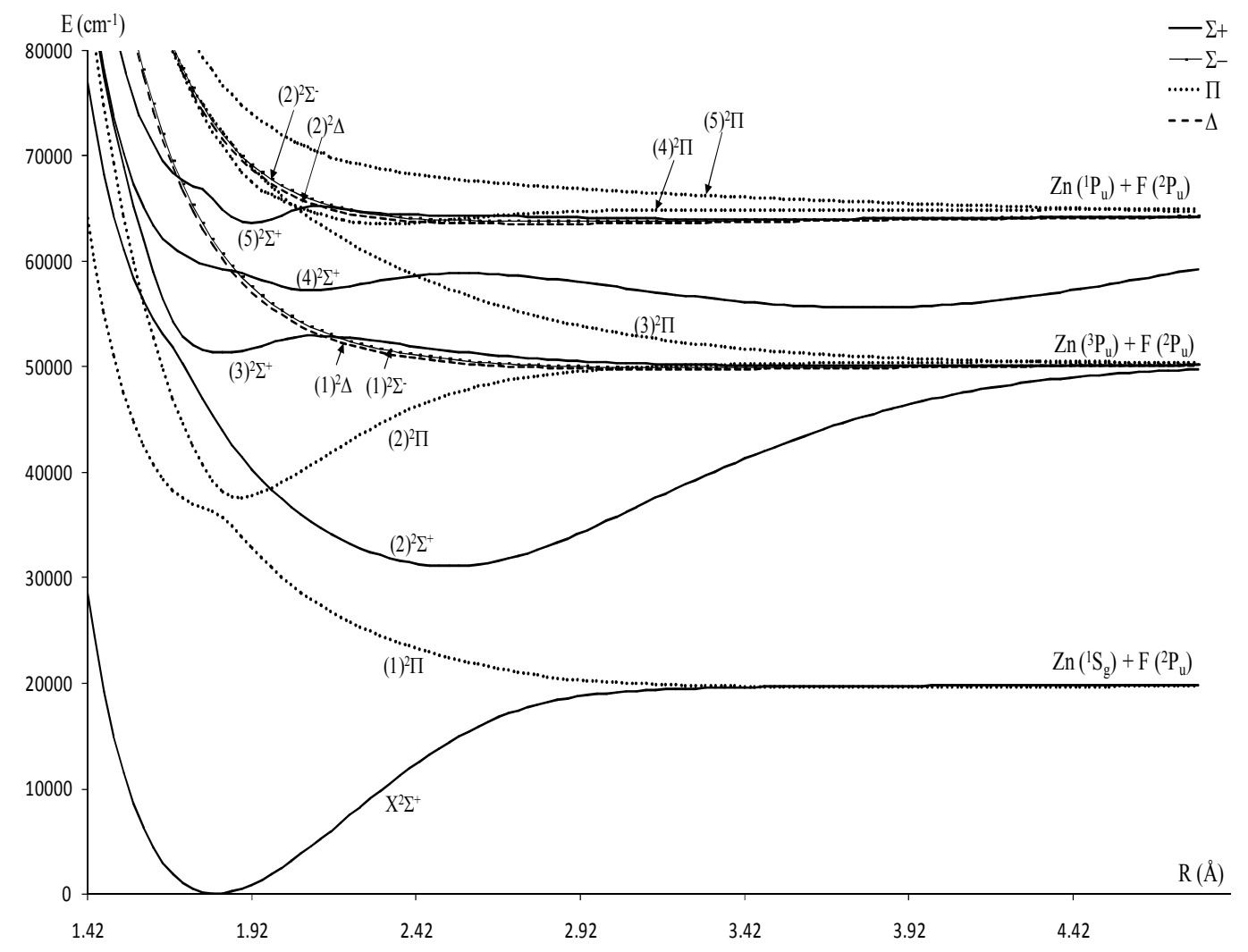

Figure (SM.1): Potential energy curves of the doublet electronic states of the ZnF molecule. 


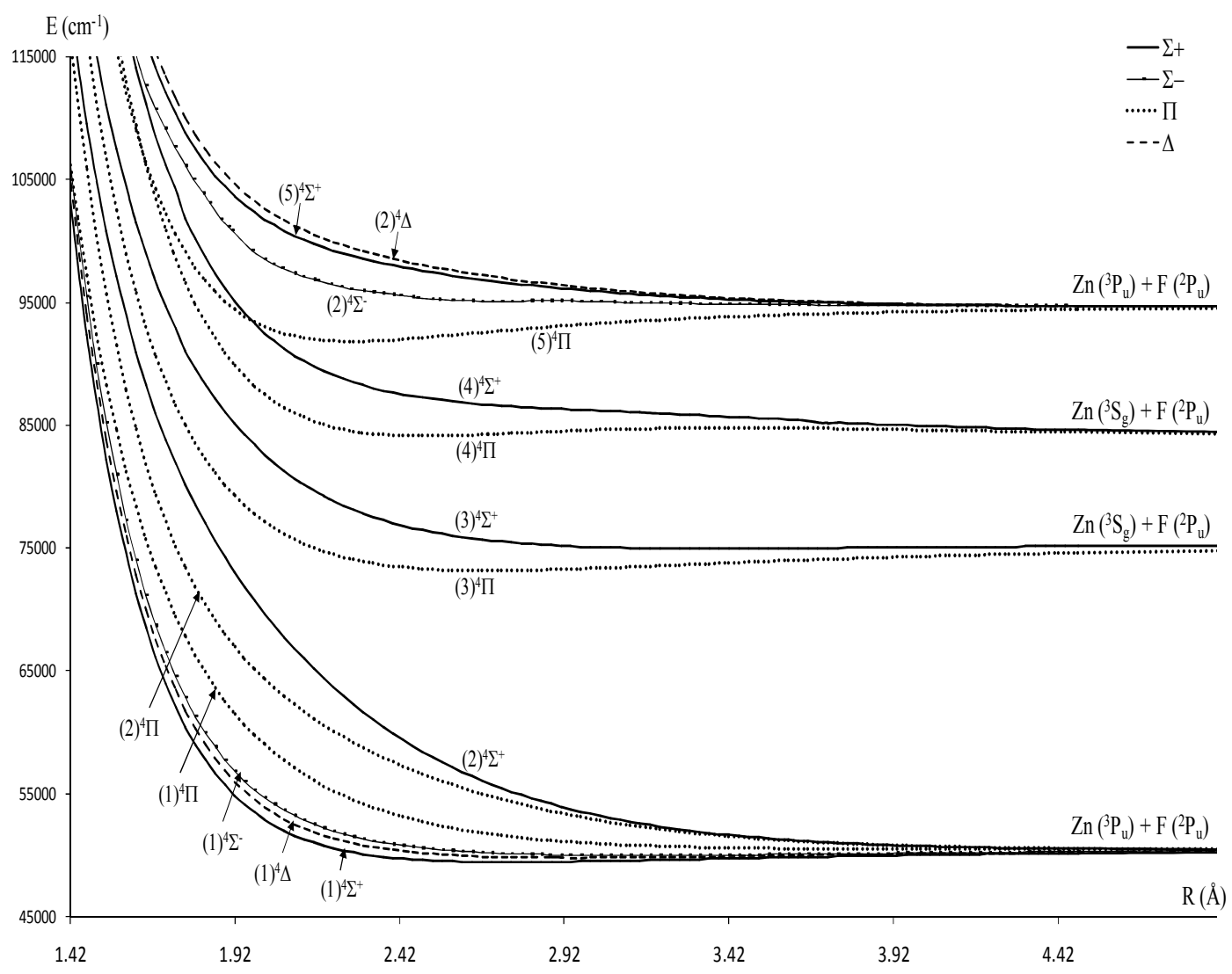

Figure (SM.2): Potential energy curves of the quartet electronic states of $\mathrm{ZnF}$. 


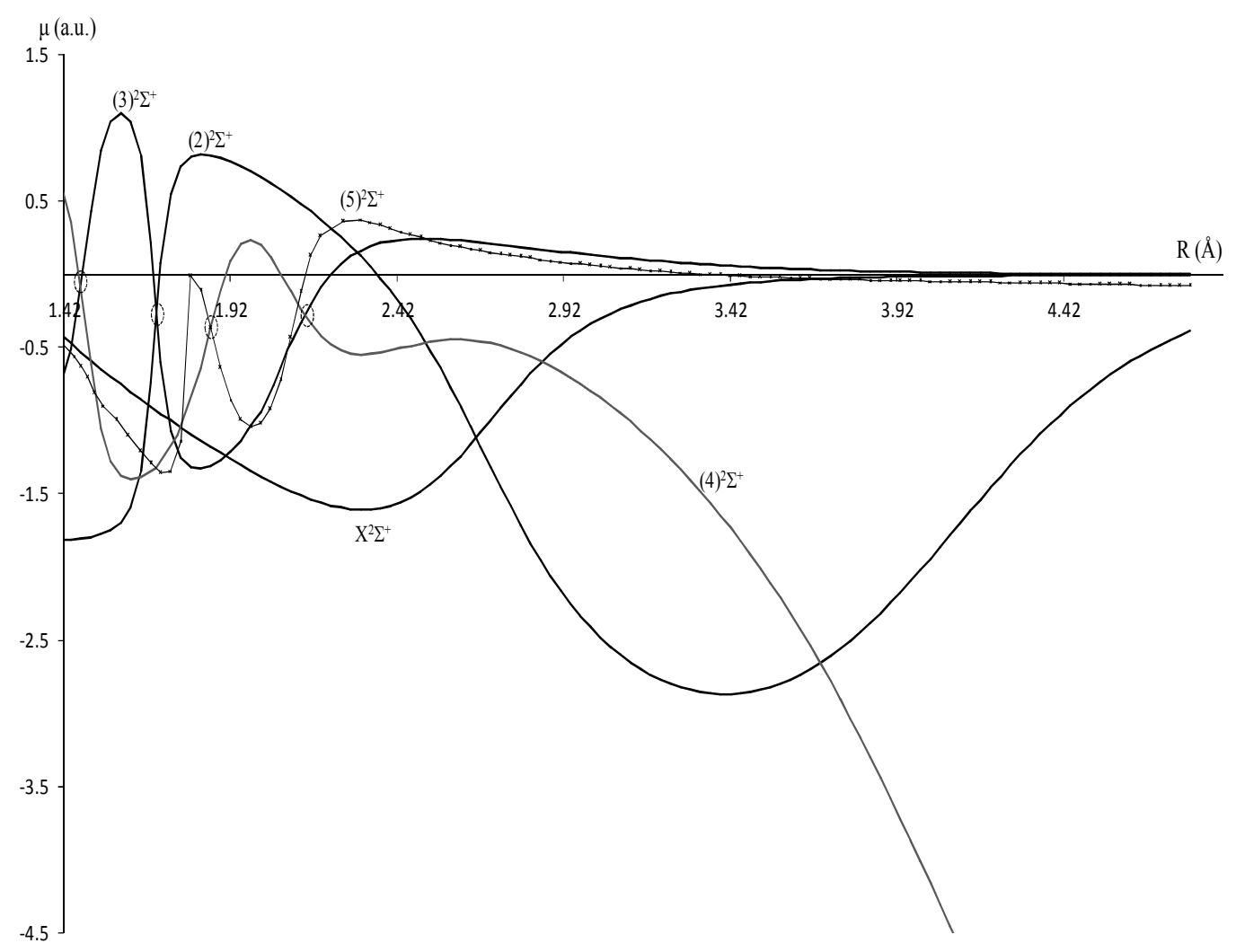

Figure (SM.3): Dipole moment curves of the ${ }^{2} \Sigma^{+}$electronic states of the $\mathrm{ZnF}$ molecule. 


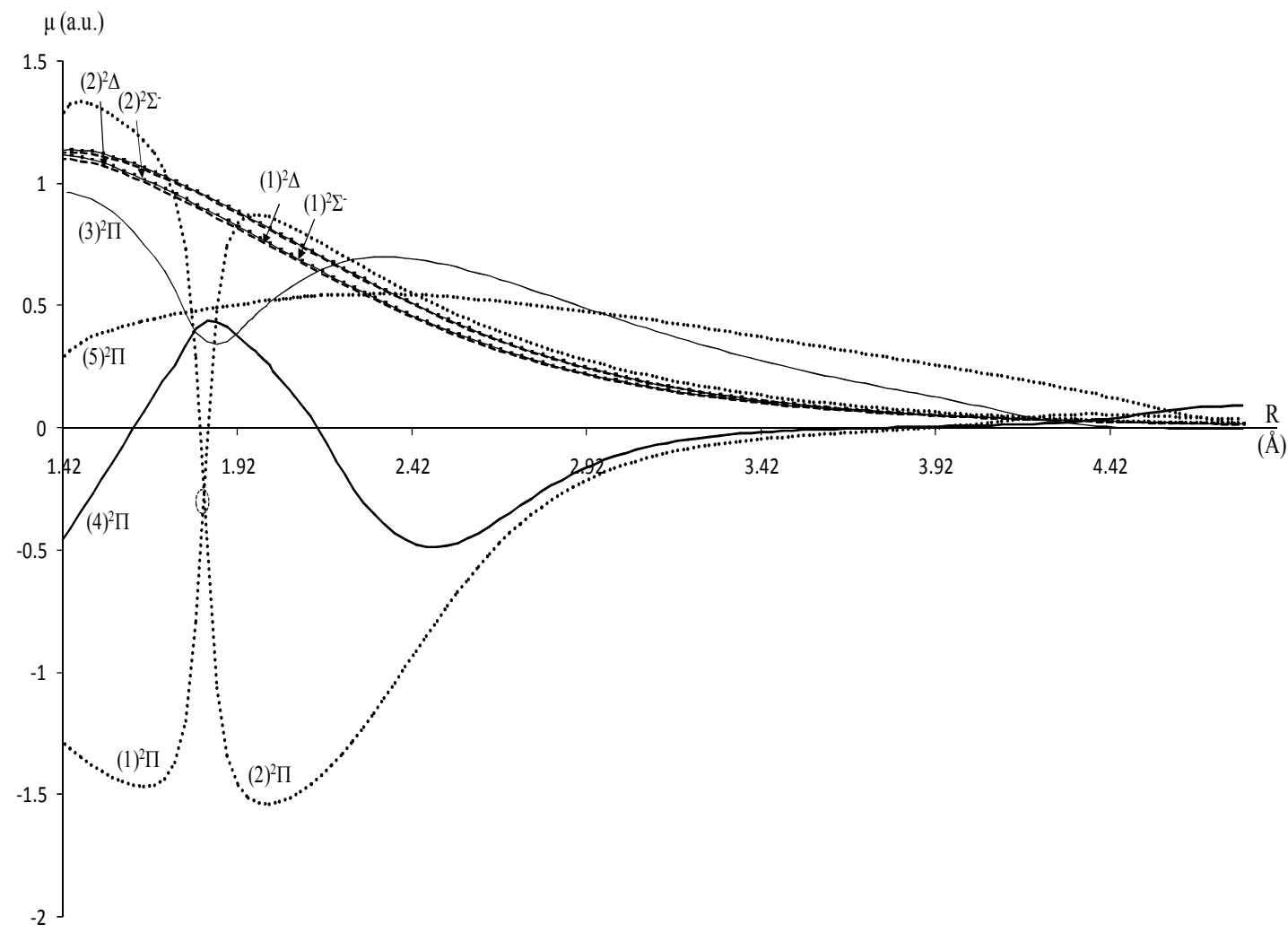

Figure (SM.4): Dipole moment curves of the ${ }^{2} \Sigma^{-},{ }^{2} \Pi$ and ${ }^{2} \Delta$ electronic states of the $\mathrm{ZnF}$ molecule. 


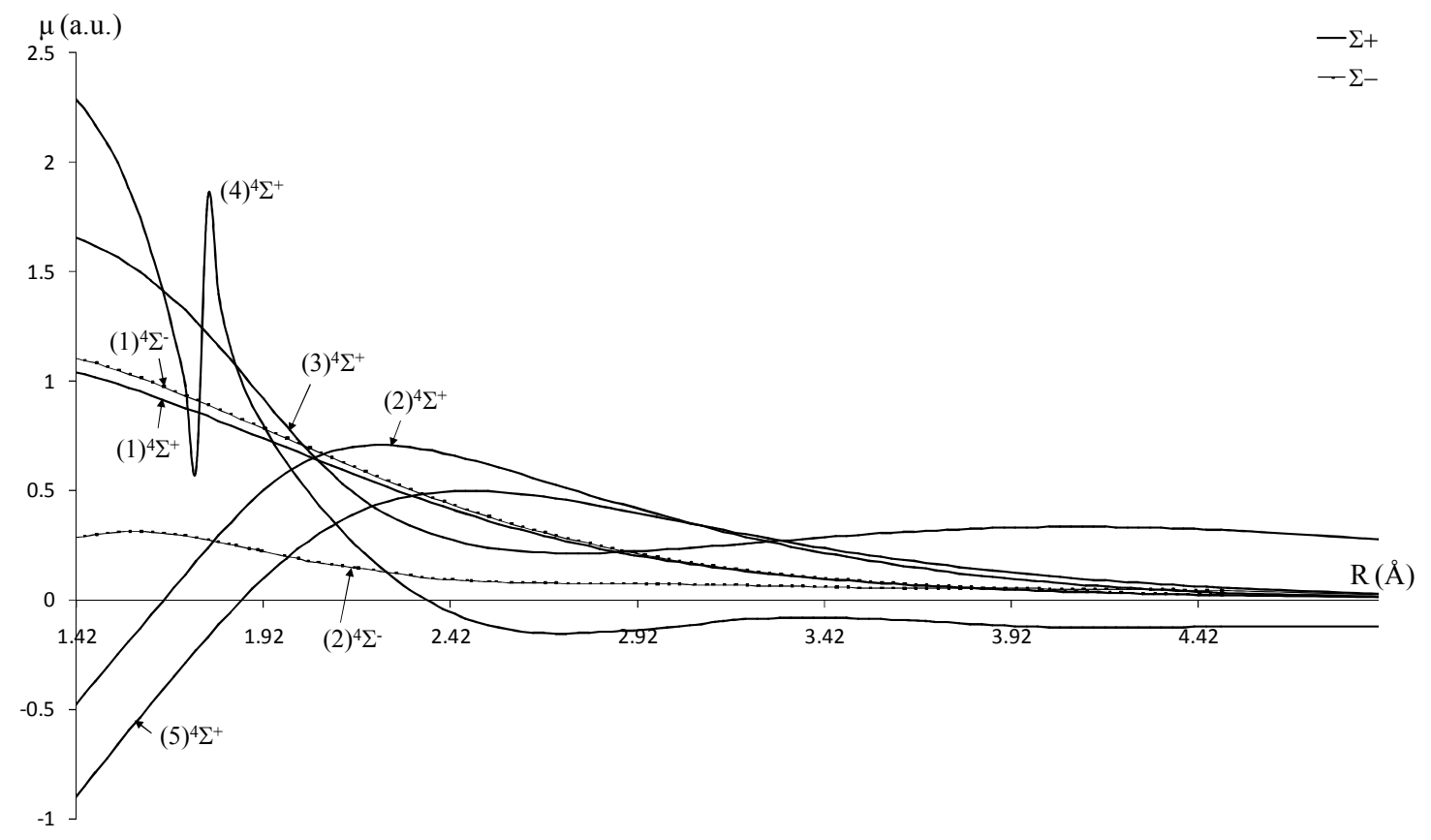

Figure (SM.5): Dipole moment curves of the ${ }^{4} \Sigma^{+}$and ${ }^{4} \Sigma^{-}$electronic states of the $\mathrm{ZnF}$ molecule. 


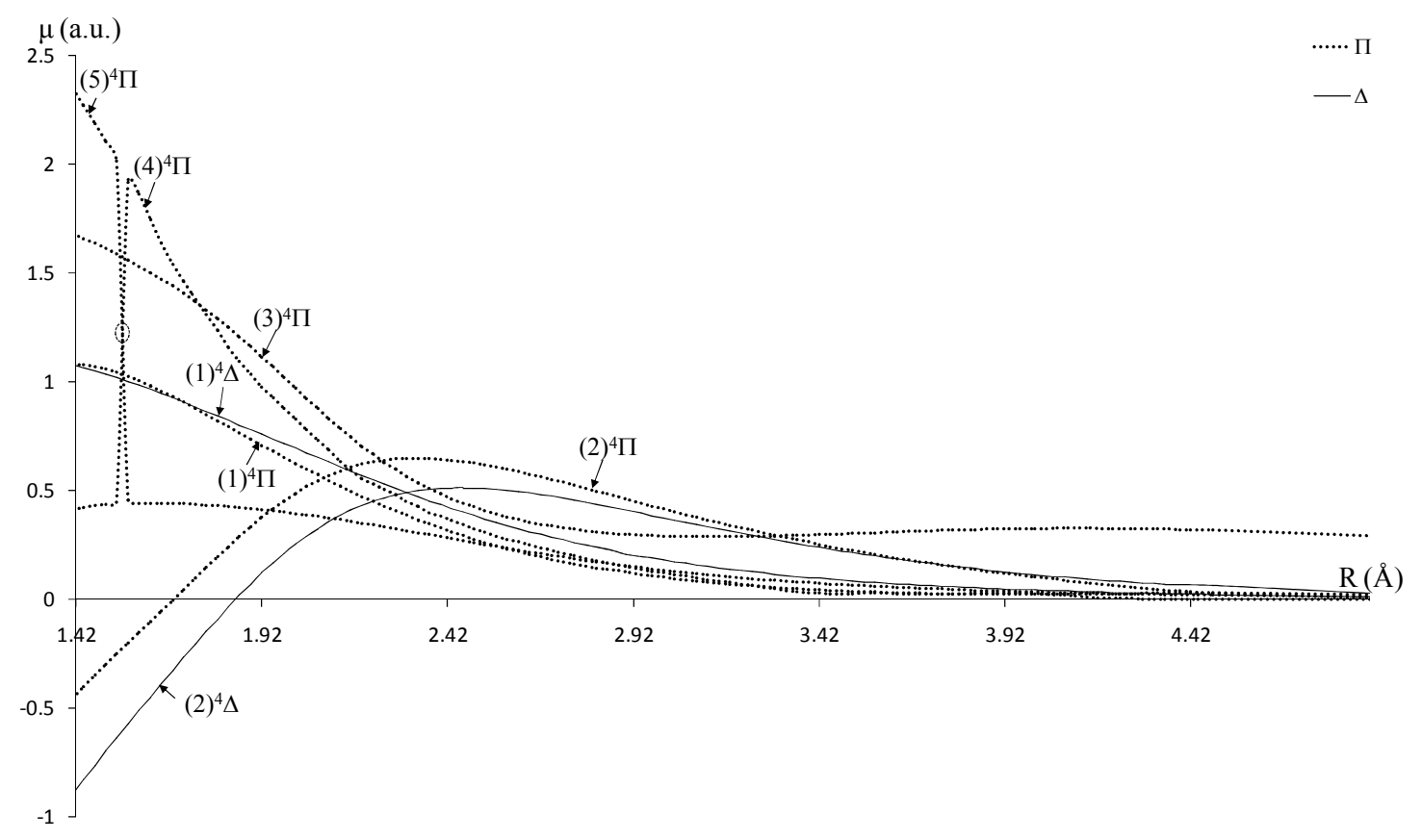

Figure (SM.6): Dipole moment curves of the ${ }^{4} \Pi$ and ${ }^{4} \Delta$ electronic states of the $\mathrm{ZnF}$ molecule. 


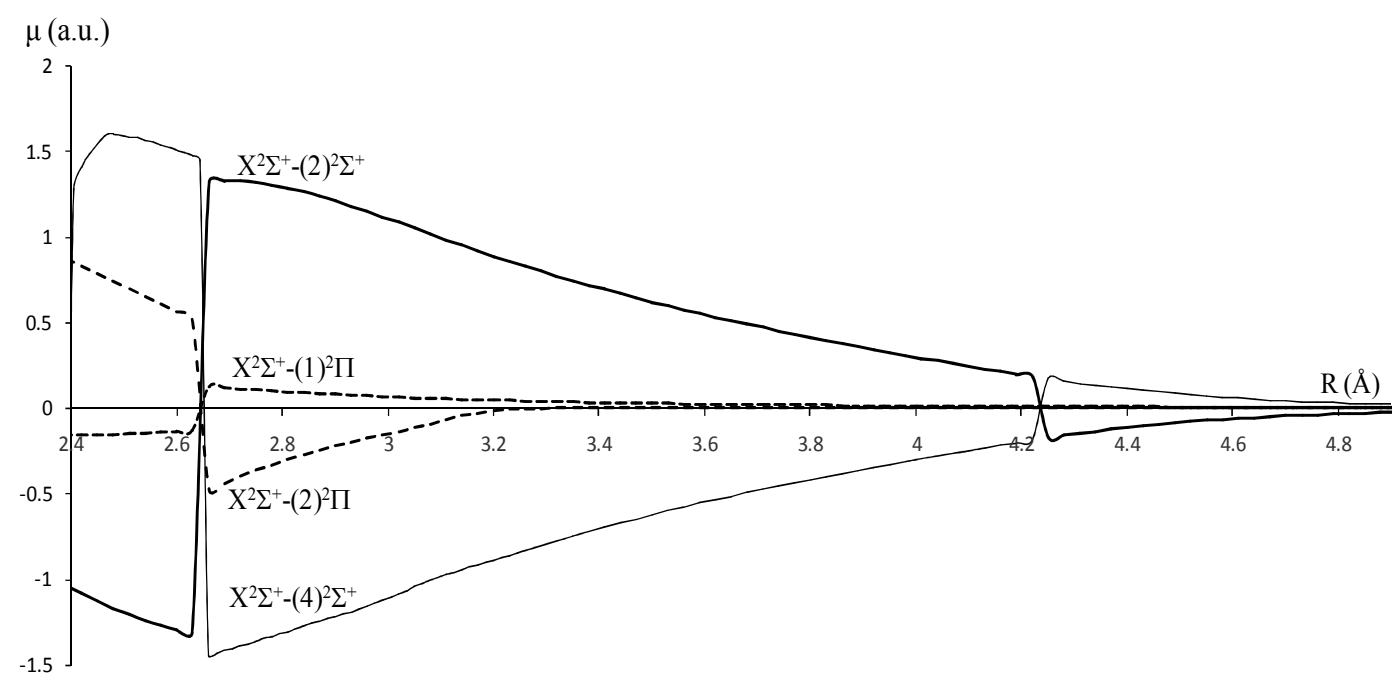

Figure (SM.7): Transition dipole moment between the ground and $(2)^{2} \Sigma^{+},(4)^{2} \Sigma^{+},(1)^{2} \Pi$, and $(2)^{2} \Pi$ electronic states of the $\mathrm{ZnF}$ molecule. 


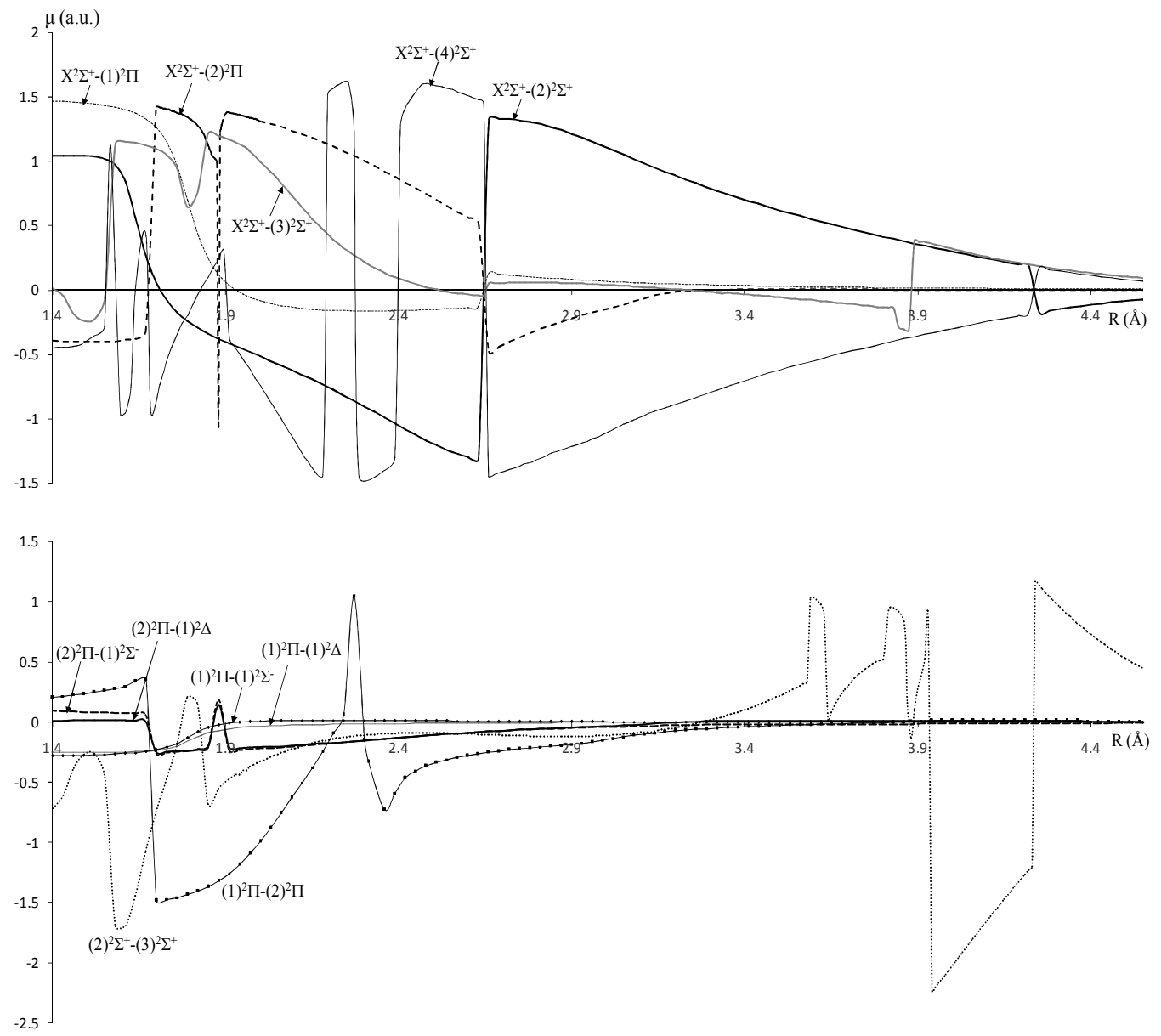

Figure (SM.8): Transition dipole moments between some doublet electronic states of the ZnF molecule. 


\section{B. Numerical data of energy and dipole moments:}

Table (SM.1): Numerical data corresponding to the potential energy E $\left(\mathrm{cm}^{-1}\right)$ and dipole moment $\mu$ (a.u.) of zinc monofluoride $\mathrm{ZnF}$ molecule as function of the internuclear distance $\mathrm{R}(\AA)$.

\begin{tabular}{|c|c|c|c|c|c|c|c|c|}
\hline \multicolumn{3}{|c|}{$\mathrm{X}^{2} \Sigma^{+}$} & \multicolumn{3}{|c|}{$(2)^{2} \Sigma^{+}$} & \multicolumn{3}{|c|}{$(3)^{2} \Sigma^{+}$} \\
\hline $\mathrm{R}(\AA)$ & $\mathrm{E}\left(\mathrm{cm}^{-1}\right)$ & $\mu$ (a.u.) & $\mathrm{R}(\AA)$ & $\mathrm{E}\left(\mathrm{cm}^{-1}\right)$ & $\mu$ (a.u.) & $\mathrm{R}(\AA)$ & $\mathrm{E}\left(\mathrm{cm}^{-1}\right)$ & $\mu$ (a.u.) \\
\hline 1.41 & 30559.4 & -0.410 & 1.41 & 78936.4 & -1.812 & 1.41 & 88577.2 & -0.759 \\
\hline 1.44 & 24399.0 & -0.473 & 1.44 & 73146.0 & -1.811 & 1.44 & 82655.5 & -0.506 \\
\hline 1.47 & 19245.3 & -0.533 & 1.47 & 68329.6 & -1.805 & 1.47 & 77560.0 & -0.064 \\
\hline 1.50 & 14930.3 & -0.590 & 1.50 & 64324.1 & -1.794 & 1.50 & 73071.4 & 0.430 \\
\hline 1.53 & 11412.4 & -0.646 & 1.53 & 61072.1 & -1.777 & 1.53 & 69017.9 & 0.842 \\
\hline 1.56 & 8538.4 & -0.701 & 1.56 & 58425.9 & -1.748 & 1.56 & 65324.7 & 1.045 \\
\hline 1.59 & 6214.0 & -0.754 & 1.59 & 56288.1 & -1.697 & 1.59 & 61991.8 & 1.100 \\
\hline 1.62 & 4360.9 & -0.805 & 1.62 & 54569.0 & -1.595 & 1.62 & 59017.7 & 1.045 \\
\hline 1.65 & 2912.5 & -0.855 & 1.65 & 53148.2 & -1.346 & 1.65 & 56425.2 & 0.815 \\
\hline 1.68 & 1811.6 & -0.905 & 1.68 & 51821.4 & -0.739 & 1.68 & 54320.4 & 0.215 \\
\hline 1.71 & 1009.2 & -0.953 & 1.71 & 50324.1 & 0.077 & 1.71 & 52883.8 & -0.599 \\
\hline 1.74 & 463.0 & -1.000 & 1.74 & 48653.8 & 0.546 & 1.74 & 52061.7 & -1.069 \\
\hline 1.77 & 137.1 & -1.047 & 1.77 & 46990.9 & 0.733 & 1.77 & 51616.3 & -1.255 \\
\hline 1.80 & 0.0 & -1.092 & 1.80 & 45421.9 & 0.801 & 1.80 & 51402.3 & -1.320 \\
\hline 1.83 & 24.8 & -1.137 & 1.83 & 43970.3 & 0.819 & 1.83 & 51347.3 & -1.331 \\
\hline 1.86 & 187.9 & -1.180 & 1.86 & 42636.2 & 0.813 & 1.86 & 51407.1 & -1.312 \\
\hline 1.89 & 468.9 & -1.223 & 1.89 & 41413.1 & 0.794 & 1.89 & 51548.9 & -1.271 \\
\hline 1.92 & 849.8 & -1.264 & 1.92 & 40292.3 & 0.768 & 1.92 & 51744.9 & -1.212 \\
\hline 1.95 & 1315.1 & -1.305 & 1.95 & 39265.7 & 0.736 & 1.95 & 52009.8 & -1.135 \\
\hline 1.98 & 1850.9 & -1.344 & 1.98 & 38325.8 & 0.700 & 1.97 & 52229.2 & -1.063 \\
\hline 2.01 & 2444.9 & -1.381 & 2.01 & 37464.9 & 0.661 & 1.99 & 52426.8 & -0.995 \\
\hline 2.04 & 3086.6 & -1.417 & 2.04 & 36676.7 & 0.619 & 2.01 & 52580.4 & -0.936 \\
\hline 2.07 & 3766.3 & -1.451 & 2.07 & 35955.6 & 0.576 & 2.03 & 52690.1 & -0.859 \\
\hline 2.10 & 4475.6 & -1.483 & 2.10 & 35296.4 & 0.530 & 2.05 & 52799.9 & -0.759 \\
\hline 2.13 & 5207.3 & -1.512 & 2.13 & 34695.9 & 0.481 & 2.07 & 52942.5 & -0.642 \\
\hline 2.16 & 5954.2 & -1.538 & 2.16 & 34149.1 & 0.429 & 2.09 & 52997.4 & -0.519 \\
\hline 2.19 & 6710.6 & -1.561 & 2.19 & 33653.1 & 0.375 & 2.11 & 52975.4 & -0.442 \\
\hline 2.22 & 7470.9 & -1.580 & 2.22 & 33205.4 & 0.319 & 2.13 & 52880.2 & -0.334 \\
\hline 2.25 & 8230.3 & -1.594 & 2.25 & 32803.7 & 0.258 & 2.16 & 52868.9 & -0.203 \\
\hline 2.28 & 8984.3 & -1.604 & 2.28 & 32446.3 & 0.194 & 2.19 & 52818.3 & -0.092 \\
\hline 2.31 & 9728.7 & -1.609 & 2.31 & 32132.0 & 0.125 & 2.22 & 52737.9 & -0.002 \\
\hline 2.34 & 10459.6 & -1.607 & 2.34 & 31859.5 & 0.052 & 2.25 & 52636.3 & 0.068 \\
\hline 2.37 & 11173.3 & -1.599 & 2.37 & 31628.3 & -0.028 & 2.28 & 52521.0 & 0.122 \\
\hline 2.40 & 11866.3 & -1.583 & 2.40 & 31438.2 & -0.113 & 2.31 & 52397.6 & 0.162 \\
\hline 2.43 & 12535.2 & -1.559 & 2.43 & 31288.4 & -0.205 & 2.34 & 52270.5 & 0.192 \\
\hline 2.46 & 13176.9 & -1.527 & 2.46 & 31179.1 & -0.304 & 2.37 & 52142.5 & 0.213 \\
\hline 2.49 & 13788.6 & -1.487 & 2.49 & 31110.8 & -0.410 & 2.40 & 52015.7 & 0.227 \\
\hline 2.52 & 14368.4 & -1.438 & 2.52 & 31083.6 & -0.524 & 2.43 & 51891.4 & 0.236 \\
\hline 2.55 & 14914.6 & -1.380 & 2.55 & 31097.8 & -0.644 & 2.46 & 51770.3 & 0.241 \\
\hline
\end{tabular}




\begin{tabular}{|c|c|c|c|c|c|c|c|c|}
\hline 2.58 & 15425.0 & -1.315 & 2.58 & 31153.4 & -0.771 & 2.49 & 51652.9 & 0.243 \\
\hline 2.61 & 15898.2 & -1.242 & 2.61 & 31250.2 & -0.903 & 2.52 & 51540.1 & 0.242 \\
\hline 2.64 & 16333.3 & -1.165 & 2.64 & 31387.4 & -1.039 & 2.55 & 51433.1 & 0.239 \\
\hline 2.67 & 16729.8 & -1.083 & 2.67 & 31564.0 & -1.177 & 2.58 & 51332.2 & 0.235 \\
\hline 2.70 & 17088.6 & -0.999 & 2.70 & 31778.3 & -1.315 & 2.61 & 51237.4 & 0.229 \\
\hline 2.73 & 17410.5 & -0.915 & 2.73 & 32028.2 & -1.452 & 2.64 & 51148.2 & 0.223 \\
\hline 2.76 & 17697.4 & -0.833 & 2.76 & 32310.9 & -1.585 & 2.67 & 51064.5 & 0.216 \\
\hline 2.79 & 17951.5 & -0.754 & 2.79 & 32623.4 & -1.713 & 2.70 & 50986.1 & 0.209 \\
\hline 2.82 & 18175.4 & -0.679 & 2.82 & 32962.3 & -1.836 & 2.73 & 50912.7 & 0.202 \\
\hline 2.85 & 18371.6 & -0.609 & 2.85 & 33324.5 & -1.951 & 2.76 & 50844.0 & 0.194 \\
\hline 2.88 & 18543.0 & -0.544 & 2.88 & 33706.3 & -2.059 & 2.79 & 50779.7 & 0.186 \\
\hline 2.91 & 18692.3 & -0.485 & 2.91 & 34104.5 & -2.159 & 2.82 & 50719.8 & 0.178 \\
\hline 2.94 & 18822.0 & -0.431 & 2.94 & 34515.9 & -2.251 & 2.85 & 50663.9 & 0.170 \\
\hline 2.97 & 18934.6 & -0.383 & 2.97 & 34937.8 & -2.335 & 2.88 & 50611.9 & 0.162 \\
\hline 3.00 & 19032.2 & -0.340 & 3.00 & 35367.3 & -2.413 & 2.91 & 50563.4 & 0.154 \\
\hline 3.03 & 19116.9 & -0.302 & 3.03 & 35802.2 & -2.482 & 2.94 & 50518.5 & 0.147 \\
\hline 3.06 & 19190.4 & -0.268 & 3.06 & 36240.5 & -2.546 & 2.97 & 50476.8 & 0.139 \\
\hline 3.09 & 19254.1 & -0.238 & 3.09 & 36680.2 & -2.602 & 3.00 & 50438.1 & 0.132 \\
\hline 3.12 & 19309.6 & -0.212 & 3.12 & 37119.8 & -2.653 & 3.03 & 50402.4 & 0.125 \\
\hline 3.15 & 19357.9 & -0.189 & 3.15 & 37557.9 & -2.697 & 3.06 & 50369.5 & 0.119 \\
\hline 3.18 & 19400.0 & -0.168 & 3.18 & 37993.4 & -2.736 & 3.09 & 50339.1 & 0.112 \\
\hline 3.21 & 19436.9 & -0.150 & 3.21 & 38425.2 & -2.770 & 3.12 & 50311.2 & 0.106 \\
\hline 3.24 & 19469.2 & -0.134 & 3.24 & 38852.3 & -2.799 & 3.15 & 50285.6 & 0.100 \\
\hline 3.27 & 19497.5 & -0.120 & 3.27 & 39274.3 & -2.822 & 3.18 & 50262.2 & 0.094 \\
\hline 3.30 & 19522.6 & -0.107 & 3.30 & 39690.3 & -2.841 & 3.21 & 50240.9 & 0.089 \\
\hline 3.33 & 19544.7 & -0.096 & 3.33 & 40099.9 & -2.855 & 3.24 & 50221.4 & 0.083 \\
\hline 3.36 & 19564.3 & -0.086 & 3.36 & 40502.5 & -2.864 & 3.27 & 50203.7 & 0.078 \\
\hline 3.39 & 19581.8 & -0.078 & 3.39 & 40897.8 & -2.868 & 3.30 & 50187.7 & 0.074 \\
\hline 3.42 & 19597.4 & -0.070 & 3.42 & 41285.4 & -2.868 & 3.33 & 50173.3 & 0.069 \\
\hline 3.45 & 19611.4 & -0.063 & 3.45 & 41665.1 & -2.863 & 3.36 & 50160.4 & 0.065 \\
\hline 3.48 & 19624.1 & -0.058 & 3.48 & 42036.5 & -2.854 & 3.39 & 50148.8 & 0.061 \\
\hline 3.51 & 19635.5 & -0.052 & 3.51 & 42399.6 & -2.840 & 3.42 & 50138.5 & 0.057 \\
\hline 3.54 & 19645.8 & -0.048 & 3.54 & 42754.1 & -2.821 & 3.45 & 50129.3 & 0.053 \\
\hline 3.57 & 19655.3 & -0.043 & 3.57 & 43099.8 & -2.797 & 3.48 & 50121.3 & 0.050 \\
\hline 3.60 & 19663.9 & -0.040 & 3.60 & 43436.5 & -2.769 & 3.51 & 50114.2 & 0.046 \\
\hline 3.63 & 19671.8 & -0.036 & 3.63 & 43764.2 & -2.737 & 3.54 & 50108.1 & 0.043 \\
\hline 3.66 & 19679.1 & -0.033 & 3.66 & 44082.7 & -2.699 & 3.57 & 50102.8 & 0.040 \\
\hline 3.69 & 19685.9 & -0.031 & 3.69 & 44391.8 & -2.657 & 3.60 & 50098.4 & 0.038 \\
\hline 3.72 & 19692.2 & -0.028 & 3.72 & 44691.5 & -2.611 & 3.63 & 50094.7 & 0.035 \\
\hline 3.75 & 19698.1 & -0.026 & 3.75 & 44981.7 & -2.560 & 3.66 & 50091.7 & 0.032 \\
\hline 3.78 & 19703.6 & -0.024 & 3.78 & 45262.2 & -2.505 & 3.69 & 50089.2 & 0.030 \\
\hline 3.81 & 19708.9 & -0.023 & 3.81 & 45533.1 & -2.446 & 3.72 & 50087.4 & 0.028 \\
\hline 3.84 & 19713.7 & -0.021 & 3.84 & 45794.3 & -2.383 & 3.75 & 50086.1 & 0.026 \\
\hline 3.87 & 19718.4 & -0.020 & 3.87 & 46045.6 & -2.316 & 3.78 & 50085.2 & 0.024 \\
\hline
\end{tabular}




\begin{tabular}{|c|c|c|c|c|c|c|c|c|}
\hline 3.90 & 19722.6 & -0.019 & 3.90 & 46287.1 & -2.247 & 3.81 & 50084.9 & 0.022 \\
\hline 3.93 & 19726.8 & -0.017 & 3.93 & 46518.9 & -2.174 & 3.84 & 50084.9 & 0.020 \\
\hline 3.96 & 19730.8 & -0.016 & 3.96 & 46740.8 & -2.099 & 3.87 & 50085.4 & 0.019 \\
\hline 3.99 & 19734.6 & -0.016 & 3.99 & 46952.9 & -2.021 & 3.90 & 50086.2 & 0.017 \\
\hline 4.02 & 19738.3 & -0.015 & 4.02 & 47155.4 & -1.942 & 3.93 & 50087.4 & 0.016 \\
\hline 4.05 & 19741.8 & -0.014 & 4.05 & 47348.4 & -1.862 & 3.96 & 50088.8 & 0.014 \\
\hline 4.08 & 19745.2 & -0.013 & 4.08 & 47531.8 & -1.781 & 3.99 & 50090.4 & 0.013 \\
\hline 4.11 & 19748.4 & -0.013 & 4.11 & 47706.0 & -1.700 & 4.02 & 50092.4 & 0.012 \\
\hline 4.14 & 19751.6 & -0.012 & 4.14 & 47871.1 & -1.619 & 4.05 & 50094.6 & 0.011 \\
\hline 4.17 & 19754.6 & -0.012 & 4.17 & 48027.3 & -1.539 & 4.08 & 50096.8 & 0.010 \\
\hline 4.20 & 19757.6 & -0.011 & 4.20 & 48174.9 & -1.459 & 4.11 & 50099.5 & 0.009 \\
\hline 4.23 & 19760.4 & -0.011 & 4.23 & 48314.2 & -1.381 & 4.14 & 50102.2 & 0.008 \\
\hline 4.26 & 19763.2 & -0.010 & 4.26 & 48445.4 & -1.305 & 4.17 & 50105.0 & 0.007 \\
\hline 4.29 & 19765.8 & -0.010 & 4.29 & 48568.7 & -1.231 & 4.20 & 50108.2 & 0.006 \\
\hline 4.32 & 19768.5 & -0.010 & 4.32 & 48684.6 & -1.159 & 4.23 & 50111.3 & 0.005 \\
\hline 4.35 & 19771.2 & -0.009 & 4.35 & 48793.4 & -1.090 & 4.26 & 50114.6 & 0.005 \\
\hline 4.38 & 19773.7 & -0.009 & 4.38 & 48895.3 & -1.024 & 4.29 & 50117.8 & 0.004 \\
\hline 4.41 & 19776.1 & -0.009 & 4.41 & 48990.8 & -0.960 & 4.32 & 50121.3 & 0.003 \\
\hline 4.44 & 19778.3 & -0.009 & 4.44 & 49080.2 & -0.900 & 4.35 & 50124.8 & 0.003 \\
\hline 4.47 & 19780.6 & -0.008 & 4.47 & 49163.7 & -0.842 & 4.38 & 50128.4 & 0.002 \\
\hline 4.50 & 19782.9 & -0.008 & 4.50 & 49241.7 & -0.787 & 4.41 & 50132.0 & 0.002 \\
\hline 4.53 & 19785.1 & -0.008 & 4.53 & 49314.5 & -0.735 & 4.44 & 50135.7 & 0.001 \\
\hline 4.56 & 19787.2 & -0.008 & 4.56 & 49382.6 & -0.686 & 4.47 & 50139.3 & 0.001 \\
\hline 4.59 & 19789.2 & -0.008 & 4.59 & 49446.1 & -0.640 & 4.50 & 50142.9 & 0.001 \\
\hline 4.62 & 19791.3 & -0.007 & 4.62 & 49505.1 & -0.597 & 4.53 & 50146.6 & 0.000 \\
\hline 4.65 & 19793.1 & -0.007 & 4.65 & 49560.4 & -0.556 & 4.56 & 50150.3 & 0.000 \\
\hline 4.68 & 19795.0 & -0.007 & 4.68 & 49611.8 & -0.518 & 4.59 & 50154.0 & 0.000 \\
\hline 4.71 & 19797.0 & -0.007 & 4.71 & 49659.8 & -0.482 & 4.62 & 50157.6 & 0.000 \\
\hline 4.74 & 19798.8 & -0.007 & 4.74 & 49704.6 & -0.448 & 4.65 & 50161.3 & 0.000 \\
\hline 4.77 & 19800.7 & -0.007 & 4.77 & 49746.6 & -0.417 & 4.68 & 50164.9 & 0.000 \\
\hline \multirow[t]{4}{*}{4.80} & 19802.7 & -0.007 & 4.80 & 49785.4 & -0.388 & 4.71 & 50168.4 & 0.000 \\
\hline & & & & & & 4.74 & 50172.0 & 0.000 \\
\hline & & & & & & 4.77 & 50175.9 & 0.000 \\
\hline & & & & & & 4.80 & 50179.3 & 0.000 \\
\hline
\end{tabular}

\begin{tabular}{|c|c|c|c|c|c|c|c|c|}
\hline \multicolumn{3}{|c|}{$(4)^{2} \Sigma^{+}$} & \multicolumn{3}{|c|}{$(5)^{2} \Sigma^{+}$} & \multicolumn{3}{|c|}{$(1)^{2} \Pi$} \\
\hline $\mathrm{R}(\AA)$ & $\mathrm{E}\left(\mathrm{cm}^{-1}\right)$ & $\mu$ (a.u.) & $\mathrm{R}(\AA)$ & $\mathrm{E}\left(\mathrm{cm}^{-1}\right)$ & $\mu$ (a.u.) & $\mathrm{R}(\AA)$ & $\mathrm{E}\left(\mathrm{cm}^{-1}\right)$ & $\mu$ (a.u.) \\
\hline 1.41 & 89817.3 & 0.633 & 1.41 & 96424.7 & -0.464 & 1.41 & 66130.5 & -1.276 \\
\hline 1.44 & 83590.0 & 0.359 & 1.45 & 89957.7 & -0.560 & 1.44 & 60151.6 & -1.314 \\
\hline 1.47 & 78240.8 & -0.105 & 1.47 & 86490.0 & -0.632 & 1.47 & 55149.8 & -1.348 \\
\hline 1.50 & 73794.1 & -0.619 & 1.49 & 83373.4 & -0.707 & 1.50 & 50945.3 & -1.379 \\
\hline 1.53 & 70211.8 & -1.052 & 1.51 & 80739.7 & -0.814 & 1.53 & 47534.1 & -1.406 \\
\hline 1.56 & 67390.0 & -1.280 & 1.54 & 77930.5 & -0.900 & 1.56 & 44751.3 & -1.429 \\
\hline 1.59 & 65181.8 & -1.375 & 1.58 & 73826.3 & -0.995 & 1.59 & 42504.0 & -1.448 \\
\hline
\end{tabular}




\begin{tabular}{|c|c|c|c|c|c|c|c|c|}
\hline 1.62 & 63468.0 & -1.400 & 1.61 & 71719.3 & -1.100 & 1.62 & 40716.1 & -1.462 \\
\hline 1.65 & 62158.9 & -1.386 & 1.65 & 69502.4 & -1.207 & 1.65 & 39324.6 & -1.468 \\
\hline 1.69 & 60997.9 & -1.330 & 1.68 & 68436.1 & -1.291 & 1.68 & 38273.9 & -1.463 \\
\hline 1.76 & 59888.8 & -1.100 & 1.71 & 67669.9 & -1.359 & 1.71 & 37509.5 & -1.436 \\
\hline 1.83 & 59291.5 & -0.641 & 1.74 & 67163.1 & -1.344 & 1.74 & 36971.2 & -1.366 \\
\hline 1.86 & 59117.2 & -0.372 & 1.77 & 66851.1 & -1.140 & 1.77 & 36589.1 & -1.196 \\
\hline 1.89 & 58888.2 & -0.110 & 1.80 & 65950.3 & -0.012 & 1.80 & 36251.5 & -0.789 \\
\hline 1.92 & 58596.3 & 0.092 & 1.83 & 64939.6 & -0.113 & 1.83 & 35707.5 & -0.083 \\
\hline 1.95 & 58269.2 & 0.204 & 1.86 & 64220.1 & -0.369 & 1.86 & 34857.8 & 0.493 \\
\hline 1.98 & 57947.8 & 0.234 & 1.89 & 63807.9 & -0.640 & 1.89 & 33840.9 & 0.747 \\
\hline 2.01 & 57667.5 & 0.198 & 1.92 & 63681.4 & -0.859 & 1.92 & 32811.5 & 0.840 \\
\hline 2.04 & 57452.5 & 0.116 & 1.95 & 63784.6 & -0.994 & 1.95 & 31832.3 & 0.870 \\
\hline 2.07 & 57315.6 & 0.006 & 1.98 & 64047.7 & -1.045 & 1.98 & 30919.3 & 0.873 \\
\hline 2.10 & 57259.4 & -0.117 & 2.01 & 64401.8 & -1.021 & 2.01 & 30076.5 & 0.863 \\
\hline 2.13 & 57279.9 & -0.235 & 2.04 & 64780.1 & -0.917 & 2.04 & 29300.3 & 0.846 \\
\hline 2.16 & 57362.0 & -0.336 & 2.07 & 65102.7 & -0.721 & 2.07 & 28585.7 & 0.826 \\
\hline 2.19 & 57490.9 & -0.418 & 2.10 & 65283.4 & -0.434 & 2.10 & 27927.8 & 0.804 \\
\hline 2.22 & 57650.7 & -0.478 & 2.13 & 65271.6 & -0.120 & 2.13 & 27321.2 & 0.780 \\
\hline 2.25 & 57826.8 & -0.518 & 2.16 & 65149.4 & 0.131 & 2.16 & 26761.0 & 0.756 \\
\hline 2.28 & 58006.9 & -0.540 & 2.19 & 65005.0 & 0.262 & 2.19 & 26242.6 & 0.731 \\
\hline 2.31 & 58181.3 & -0.549 & 2.26 & 64650.3 & 0.358 & 2.22 & 25762.1 & 0.707 \\
\hline 2.34 & 58342.9 & -0.546 & 2.31 & 64573.4 & 0.372 & 2.25 & 25316.0 & 0.682 \\
\hline 2.37 & 58486.8 & -0.537 & 2.34 & 64512.3 & 0.355 & 2.28 & 24901.0 & 0.658 \\
\hline 2.40 & 58610.0 & -0.522 & 2.37 & 64468.3 & 0.334 & 2.31 & 24514.3 & 0.635 \\
\hline 2.43 & 58711.2 & -0.506 & 2.40 & 64436.9 & 0.312 & 2.34 & 24153.4 & 0.611 \\
\hline 2.46 & 58790.2 & -0.489 & 2.43 & 64415.6 & 0.291 & 2.37 & 23816.0 & 0.588 \\
\hline 2.49 & 58847.2 & -0.474 & 2.46 & 64402.5 & 0.269 & 2.40 & 23499.9 & 0.566 \\
\hline 2.52 & 58883.1 & -0.462 & 2.49 & 64396.1 & 0.250 & 2.43 & 23203.5 & 0.545 \\
\hline 2.55 & 58899.0 & -0.453 & 2.52 & 64392.9 & 0.231 & 2.46 & 22925.1 & 0.524 \\
\hline 2.58 & 58896.1 & -0.448 & 2.55 & 64388.0 & 0.215 & 2.49 & 22663.1 & 0.503 \\
\hline 2.61 & 58876.2 & -0.447 & 2.58 & 64379.9 & 0.200 & 2.52 & 22416.6 & 0.484 \\
\hline 2.64 & 58840.6 & -0.450 & 2.61 & 64369.1 & 0.186 & 2.55 & 22184.6 & 0.464 \\
\hline 2.67 & 58791.2 & -0.458 & 2.64 & 64352.9 & 0.173 & 2.58 & 21966.0 & 0.446 \\
\hline 2.70 & 58729.3 & -0.470 & 2.67 & 64337.0 & 0.161 & 2.61 & 21759.7 & 0.428 \\
\hline 2.73 & 58656.5 & -0.486 & 2.70 & 64319.7 & 0.150 & 2.64 & 21564.8 & 0.411 \\
\hline 2.76 & 58574.0 & -0.507 & 2.73 & 64300.7 & 0.139 & 2.67 & 21380.6 & 0.394 \\
\hline 2.79 & 58483.6 & -0.531 & 2.76 & 64281.2 & 0.129 & 2.70 & 21206.2 & 0.378 \\
\hline 2.82 & 58385.5 & -0.559 & 2.79 & 64260.8 & 0.119 & 2.73 & 21040.9 & 0.362 \\
\hline 2.85 & 58281.3 & -0.591 & 2.82 & 64240.1 & 0.110 & 2.76 & 20884.4 & 0.348 \\
\hline 2.88 & 58172.2 & -0.625 & 2.85 & 64219.5 & 0.101 & 2.79 & 20735.6 & 0.333 \\
\hline 2.91 & 58058.9 & -0.663 & 2.88 & 64199.9 & 0.092 & 2.82 & 20594.3 & 0.319 \\
\hline 2.94 & 57942.4 & -0.704 & 2.91 & 64180.4 & 0.084 & 2.85 & 20459.8 & 0.306 \\
\hline 2.97 & 57823.6 & -0.748 & 2.94 & 64161.9 & 0.076 & 2.88 & 20354.8 & 0.293 \\
\hline 3.00 & 57703.3 & -0.795 & 2.97 & 64144.4 & 0.068 & 2.91 & 20282.8 & 0.281 \\
\hline
\end{tabular}




\begin{tabular}{|c|c|c|c|c|c|c|c|c|}
\hline 3.03 & 57582.1 & -0.844 & 3.00 & 64128.0 & 0.061 & 2.94 & 20216.4 & 0.269 \\
\hline 3.06 & 57460.8 & -0.896 & 3.03 & 64112.8 & 0.054 & 2.97 & 20155.2 & 0.258 \\
\hline 3.09 & 57339.9 & -0.951 & 3.06 & 64099.0 & 0.047 & 3.00 & 20099.0 & 0.247 \\
\hline 3.12 & 57220.2 & -1.009 & 3.09 & 64086.6 & 0.041 & 3.03 & 20047.3 & 0.237 \\
\hline 3.15 & 57101.9 & -1.069 & 3.12 & 64075.3 & 0.035 & 3.06 & 19999.9 & 0.227 \\
\hline 3.18 & 56985.9 & -1.132 & 3.15 & 64065.4 & 0.029 & 3.09 & 19956.4 & 0.217 \\
\hline 3.21 & 56872.3 & -1.197 & 3.18 & 64056.9 & 0.024 & 3.12 & 19916.7 & 0.208 \\
\hline 3.24 & 56761.5 & -1.265 & 3.21 & 64049.6 & 0.019 & 3.15 & 19880.5 & 0.199 \\
\hline 3.27 & 56654.2 & -1.336 & 3.24 & 64043.3 & 0.014 & 3.18 & 19847.4 & 0.191 \\
\hline 3.30 & 56550.6 & -1.409 & 3.27 & 64038.1 & 0.010 & 3.21 & 19817.4 & 0.182 \\
\hline 3.33 & 56451.1 & -1.486 & 3.30 & 64034.2 & 0.005 & 3.24 & 19790.2 & 0.175 \\
\hline 3.36 & 56355.9 & -1.565 & 3.33 & 64031.2 & 0.002 & 3.27 & 19765.6 & 0.167 \\
\hline 3.39 & 56265.4 & -1.648 & 3.36 & 64029.4 & -0.002 & 3.30 & 19743.4 & 0.160 \\
\hline 3.42 & 56179.8 & -1.733 & 3.39 & 64028.3 & -0.006 & 3.33 & 19723.4 & 0.153 \\
\hline 3.45 & 56099.4 & -1.822 & 3.42 & 64027.8 & -0.009 & 3.36 & 19705.5 & 0.147 \\
\hline 3.48 & 56024.6 & -1.915 & 3.45 & 64028.1 & -0.012 & 3.39 & 19689.5 & 0.140 \\
\hline 3.51 & 55955.5 & -2.010 & 3.48 & 64029.1 & -0.015 & 3.42 & 19675.3 & 0.134 \\
\hline 3.54 & 55892.3 & -2.110 & 3.51 & 64030.7 & -0.018 & 3.45 & 19662.7 & 0.129 \\
\hline 3.57 & 55835.4 & -2.212 & 3.54 & 64032.8 & -0.020 & 3.48 & 19651.6 & 0.123 \\
\hline 3.60 & 55784.9 & -2.319 & 3.57 & 64035.4 & -0.023 & 3.51 & 19642.0 & 0.118 \\
\hline 3.63 & 55741.1 & -2.429 & 3.60 & 64038.8 & -0.025 & 3.54 & 19633.6 & 0.113 \\
\hline 3.66 & 55704.1 & -2.543 & 3.63 & 64042.3 & -0.027 & 3.57 & 19626.4 & 0.108 \\
\hline 3.69 & 55674.1 & -2.660 & 3.66 & 64046.3 & -0.029 & 3.60 & 19620.2 & 0.103 \\
\hline 3.72 & 55651.4 & -2.782 & 3.69 & 64050.6 & -0.031 & 3.63 & 19615.3 & 0.099 \\
\hline 3.75 & 55636.2 & -2.907 & 3.72 & 64055.2 & -0.033 & 3.66 & 19610.9 & 0.094 \\
\hline 3.78 & 55628.5 & -3.035 & 3.75 & 64060.0 & -0.035 & 3.69 & 19607.5 & 0.090 \\
\hline 3.81 & 55628.9 & -3.167 & 3.78 & 64065.1 & -0.037 & 3.72 & 19604.9 & 0.087 \\
\hline 3.84 & 55636.6 & -3.301 & 3.81 & 64070.4 & -0.038 & 3.75 & 19603.2 & 0.083 \\
\hline 3.87 & 55652.8 & -3.439 & 3.84 & 64075.8 & -0.040 & 3.78 & 19601.8 & 0.079 \\
\hline 3.90 & 55676.5 & -3.579 & 3.87 & 64081.5 & -0.041 & 3.81 & 19601.2 & 0.076 \\
\hline 3.93 & 55709.2 & -3.722 & 3.90 & 64087.4 & -0.043 & 3.83 & 19601.0 & 0.073 \\
\hline 3.96 & 55749.5 & -3.866 & 3.93 & 64093.5 & -0.044 & 3.87 & 19601.4 & 0.070 \\
\hline 3.99 & 55797.4 & -4.012 & 3.96 & 64099.5 & -0.045 & 3.90 & 19602.4 & 0.067 \\
\hline 4.02 & 55854.0 & -4.158 & 3.99 & 64105.5 & -0.047 & 3.93 & 19603.7 & 0.064 \\
\hline 4.05 & 55919.2 & -4.306 & 4.02 & 64111.9 & -0.048 & 3.96 & 19605.4 & 0.061 \\
\hline 4.08 & 55991.2 & -4.453 & 4.05 & 64118.3 & -0.049 & 3.99 & 19607.5 & 0.058 \\
\hline 4.11 & 56071.5 & -4.600 & 4.08 & 64124.6 & -0.051 & 4.02 & 19609.8 & 0.056 \\
\hline 4.14 & 56159.4 & -4.747 & 4.11 & 64131.1 & -0.052 & 4.05 & 19612.6 & 0.053 \\
\hline 4.17 & 56254.6 & -4.891 & 4.14 & 64137.6 & -0.053 & 4.08 & 19615.3 & 0.051 \\
\hline 4.20 & 56356.8 & -5.035 & 4.17 & 64144.2 & -0.054 & 4.11 & 19618.4 & 0.049 \\
\hline 4.23 & 56465.4 & -5.175 & 4.20 & 64151.2 & -0.055 & 4.14 & 19621.7 & 0.047 \\
\hline 4.26 & 56581.3 & -5.314 & 4.23 & 64157.9 & -0.057 & 4.17 & 19625.2 & 0.045 \\
\hline 4.29 & 56702.2 & -5.449 & 4.26 & 64163.9 & -0.057 & 4.20 & 19629.1 & 0.043 \\
\hline 4.32 & 56829.3 & -5.582 & 4.29 & 64170.9 & -0.059 & 4.23 & 19632.8 & 0.041 \\
\hline
\end{tabular}




\begin{tabular}{|c|c|c|c|c|c|c|c|c|}
\hline 4.35 & 56961.5 & -5.710 & 4.32 & 64177.6 & -0.060 & 4.26 & 19636.7 & 0.039 \\
\hline 4.38 & 57098.7 & -5.836 & 4.35 & 64184.2 & -0.061 & 4.29 & 19640.7 & 0.037 \\
\hline 4.41 & 57240.3 & -5.957 & 4.38 & 64191.2 & -0.062 & 4.32 & 19644.8 & 0.036 \\
\hline 4.44 & 57385.9 & -6.075 & 4.41 & 64197.7 & -0.063 & 4.35 & 19649.0 & 0.034 \\
\hline 4.47 & 57535.0 & -6.189 & 4.44 & 64204.3 & -0.065 & 4.38 & 19653.2 & 0.033 \\
\hline 4.50 & 57687.5 & -6.298 & 4.47 & 64210.7 & -0.066 & 4.41 & 19657.4 & 0.031 \\
\hline 4.53 & 57842.7 & -6.404 & 4.50 & 64217.0 & -0.067 & 4.44 & 19661.7 & 0.030 \\
\hline 4.56 & 57999.9 & -6.505 & 4.53 & 64223.3 & -0.068 & 4.47 & 19666.0 & 0.029 \\
\hline 4.59 & 58159.5 & -6.603 & 4.56 & 64229.5 & -0.069 & 4.50 & 19670.3 & 0.027 \\
\hline 4.62 & 58320.6 & -6.696 & 4.59 & 64236.5 & -0.070 & 4.53 & 19674.5 & 0.026 \\
\hline 4.65 & 58483.0 & -6.785 & 4.62 & 64242.4 & -0.072 & 4.56 & 19678.8 & 0.025 \\
\hline 4.68 & 58646.5 & -6.870 & 4.65 & 64248.7 & -0.073 & 4.59 & 19682.9 & 0.024 \\
\hline 4.71 & 58811.0 & -6.951 & 4.68 & 64254.8 & -0.075 & 4.62 & 19687.1 & 0.023 \\
\hline 4.74 & 58976.0 & -7.028 & 4.71 & 64260.6 & -0.076 & 4.65 & 19691.2 & 0.022 \\
\hline 4.77 & 59143.8 & -7.101 & 4.74 & 64266.4 & -0.077 & 4.68 & 19695.3 & 0.021 \\
\hline \multirow[t]{4}{*}{4.80} & 59308.5 & -7.170 & 4.77 & 64272.2 & -0.078 & 4.71 & 19699.2 & 0.020 \\
\hline & & & 4.80 & 64278.2 & -0.080 & 4.74 & 19703.2 & 0.019 \\
\hline & & & & & & 4.77 & 19707.1 & 0.018 \\
\hline & & & & & & 4.80 & 19710.9 & 0.018 \\
\hline
\end{tabular}

\begin{tabular}{|c|c|c|c|c|c|c|c|c|}
\hline \multicolumn{3}{|c|}{$(2)^{2} \Pi$} & \multicolumn{3}{|c|}{$(3)^{2} \Pi$} & \multicolumn{3}{|c|}{$(4)^{2} \Pi$} \\
\hline $\mathrm{R}(\AA)$ & $\mathrm{E}\left(\mathrm{cm}^{-1}\right)$ & $\mu$ (a.u.) & $\mathrm{R}(\AA)$ & $\mathrm{E}\left(\mathrm{cm}^{-1}\right)$ & $\mu$ (a.u.) & $\mathrm{R}(\AA)$ & $\mathrm{E}\left(\mathrm{cm}^{-1}\right)$ & $\mu$ (a.u.) \\
\hline 1.41 & 87887.7 & 1.268 & 1.43 & 108735.4 & 0.962 & 1.41 & 119740.9 & -0.478 \\
\hline 1.44 & 81036.6 & 1.327 & 1.44 & 107244.3 & 0.963 & 1.44 & 112654.1 & -0.412 \\
\hline 1.47 & 74797.4 & 1.336 & 1.46 & 103026.6 & 0.953 & 1.47 & 106423.8 & -0.344 \\
\hline 1.50 & 69229.2 & 1.326 & 1.50 & 96673.6 & 0.938 & 1.50 & 100951.4 & -0.270 \\
\hline 1.53 & 64302.4 & 1.306 & 1.53 & 92874.0 & 0.911 & 1.53 & 96180.5 & -0.208 \\
\hline 1.56 & 59948.0 & 1.280 & 1.56 & 89211.2 & 0.886 & 1.56 & 92026.7 & -0.145 \\
\hline 1.59 & 56096.6 & 1.250 & 1.59 & 85982.0 & 0.848 & 1.59 & 88378.0 & -0.075 \\
\hline 1.62 & 52687.4 & 1.215 & 1.62 & 83219.8 & 0.802 & 1.62 & 85202.5 & -0.001 \\
\hline 1.65 & 49664.5 & 1.174 & 1.65 & 80695.8 & 0.753 & 1.65 & 82298.0 & 0.065 \\
\hline 1.68 & 46982.1 & 1.123 & 1.68 & 78437.2 & 0.700 & 1.68 & 79971.6 & 0.134 \\
\hline 1.71 & 44603.0 & 1.052 & 1.71 & 76532.8 & 0.643 & 1.70 & 78193.8 & 0.190 \\
\hline 1.74 & 42503.5 & 0.941 & 1.74 & 74432.7 & 0.562 & 1.74 & 75801.6 & 0.260 \\
\hline 1.77 & 40680.6 & 0.731 & 1.77 & 73193.5 & 0.466 & 1.77 & 73870.2 & 0.334 \\
\hline 1.80 & 39169.8 & 0.287 & 1.80 & 71819.1 & 0.389 & 1.80 & 72333.9 & 0.409 \\
\hline 1.83 & 38105.1 & -0.454 & 1.83 & 70525.2 & 0.354 & 1.83 & 70973.1 & 0.437 \\
\hline 1.86 & 37623.1 & -1.062 & 1.86 & 69336.0 & 0.343 & 1.86 & 69722.1 & 0.435 \\
\hline 1.89 & 37578.9 & -1.345 & 1.89 & 68163.8 & 0.355 & 1.89 & 68515.0 & 0.413 \\
\hline 1.92 & 37772.3 & -1.464 & 1.92 & 67174.9 & 0.391 & 1.92 & 67483.5 & 0.373 \\
\hline 1.95 & 38104.0 & -1.515 & 1.93 & 66228.7 & 0.406 & 1.95 & 66770.0 & 0.334 \\
\hline 1.98 & 38524.4 & -1.535 & 1.94 & 65927.4 & 0.420 & 1.96 & 66626.5 & 0.324 \\
\hline 2.01 & 39007.8 & -1.538 & 1.95 & 65633.2 & 0.434 & 1.97 & 66476.4 & 0.313 \\
\hline 2.04 & 39535.3 & -1.530 & 1.96 & 65345.8 & 0.448 & 1.98 & 66323.6 & 0.300 \\
\hline
\end{tabular}




\begin{tabular}{|c|c|c|c|c|c|c|c|c|}
\hline 2.07 & 40092.5 & -1.513 & 1.97 & 65065.1 & 0.462 & 1.99 & 66170.7 & 0.286 \\
\hline 2.10 & 40668.8 & -1.489 & 1.98 & 64790.7 & 0.475 & 2.00 & 66019.7 & 0.271 \\
\hline 2.13 & 41254.9 & -1.458 & 1.99 & 64473.0 & 0.488 & 2.01 & 65871.8 & 0.255 \\
\hline 2.16 & 41842.0 & -1.421 & 2.00 & 63724.5 & 0.501 & 2.02 & 65727.8 & 0.233 \\
\hline 2.19 & 42423.6 & -1.379 & 2.01 & 63010.1 & 0.515 & 2.07 & 65084.2 & 0.153 \\
\hline 2.22 & 42993.4 & -1.332 & 2.04 & 62334.1 & 0.543 & 2.08 & 64972.3 & 0.135 \\
\hline 2.25 & 43547.4 & -1.280 & 2.07 & 61694.5 & 0.576 & 2.09 & 64866.0 & 0.116 \\
\hline 2.28 & 44081.0 & -1.225 & 2.10 & 61140.0 & 0.604 & 2.10 & 64765.4 & 0.098 \\
\hline 2.31 & 44591.1 & -1.166 & 2.13 & 60720.4 & 0.628 & 2.11 & 64671.2 & 0.080 \\
\hline 2.34 & 45074.9 & -1.105 & 2.16 & 60370.0 & 0.649 & 2.12 & 64581.8 & 0.062 \\
\hline 2.37 & 45531.0 & -1.042 & 2.19 & 60030.1 & 0.666 & 2.13 & 64497.8 & 0.044 \\
\hline 2.40 & 45957.8 & -0.978 & 2.22 & 59730.1 & 0.679 & 2.18 & 64062.6 & -0.073 \\
\hline 2.43 & 46354.8 & -0.914 & 2.25 & 59430.0 & 0.689 & 2.20 & 63898.7 & -0.134 \\
\hline 2.46 & 46721.8 & -0.851 & 2.28 & 59189.9 & 0.695 & 2.23 & 63768.0 & -0.193 \\
\hline 2.49 & 47059.3 & -0.789 & 2.31 & 58900.3 & 0.699 & 2.25 & 63673.6 & -0.250 \\
\hline 2.52 & 47368.9 & -0.729 & 2.34 & 58580.7 & 0.700 & 2.28 & 63612.5 & -0.304 \\
\hline 2.55 & 47653.1 & -0.671 & 2.37 & 58320.4 & 0.699 & 2.31 & 63582.1 & -0.352 \\
\hline 2.58 & 47913.0 & -0.617 & 2.40 & 58040.2 & 0.696 & 2.34 & 63579.4 & -0.394 \\
\hline 2.61 & 48150.0 & -0.565 & 2.43 & 57758.0 & 0.691 & 2.37 & 63601.1 & -0.430 \\
\hline 2.64 & 48365.5 & -0.516 & 2.46 & 57402.4 & 0.684 & 2.40 & 63643.6 & -0.457 \\
\hline 2.67 & 48560.6 & -0.471 & 2.49 & 57062.0 & 0.676 & 2.43 & 63702.5 & -0.476 \\
\hline 2.70 & 48737.0 & -0.429 & 2.52 & 56735.9 & 0.667 & 2.46 & 63774.5 & -0.487 \\
\hline 2.73 & 48896.2 & -0.391 & 2.55 & 56423.7 & 0.657 & 2.49 & 63855.8 & -0.489 \\
\hline 2.76 & 49040.1 & -0.355 & 2.58 & 56124.8 & 0.645 & 2.52 & 63942.7 & -0.483 \\
\hline 2.79 & 49169.3 & -0.323 & 2.61 & 55838.8 & 0.633 & 2.55 & 64032.3 & -0.470 \\
\hline 2.82 & 49285.6 & -0.293 & 2.64 & 55565.3 & 0.621 & 2.58 & 64121.0 & -0.452 \\
\hline 2.85 & 49390.2 & -0.266 & 2.67 & 55303.7 & 0.608 & 2.61 & 64207.1 & -0.429 \\
\hline 2.88 & 49484.3 & -0.242 & 2.70 & 55053.7 & 0.594 & 2.64 & 64289.2 & -0.403 \\
\hline 2.91 & 49569.2 & -0.219 & 2.73 & 54814.7 & 0.580 & 2.67 & 64365.9 & -0.375 \\
\hline 2.94 & 49645.4 & -0.199 & 2.76 & 54586.5 & 0.566 & 2.70 & 64436.7 & -0.347 \\
\hline 2.97 & 49714.4 & -0.181 & 2.79 & 54368.7 & 0.552 & 2.73 & 64501.2 & -0.318 \\
\hline 3.00 & 49776.4 & -0.164 & 2.82 & 54161.2 & 0.537 & 2.76 & 64560.5 & -0.289 \\
\hline 3.03 & 49832.9 & -0.150 & 2.85 & 53963.0 & 0.523 & 2.79 & 64612.1 & -0.261 \\
\hline 3.06 & 49884.8 & -0.136 & 2.88 & 53773.9 & 0.509 & 2.82 & 64657.6 & -0.235 \\
\hline 3.09 & 49933.0 & -0.124 & 2.91 & 53593.9 & 0.494 & 2.85 & 64697.6 & -0.211 \\
\hline 3.12 & 49977.8 & -0.113 & 2.94 & 53422.0 & 0.480 & 2.88 & 64732.3 & -0.188 \\
\hline 3.15 & 50019.0 & -0.103 & 2.97 & 108735.4 & 0.465 & 2.91 & 64763.1 & -0.168 \\
\hline 3.18 & 50056.6 & -0.093 & 3.00 & 107244.3 & 0.451 & 2.94 & 64788.9 & -0.149 \\
\hline 3.21 & 50090.9 & -0.085 & 3.03 & 53258.0 & 0.437 & 2.97 & 64811.1 & -0.132 \\
\hline 3.24 & 50122.0 & -0.078 & 3.06 & 53101.9 & 0.423 & 3.00 & 64829.7 & -0.116 \\
\hline 3.27 & 50150.4 & -0.071 & 3.09 & 52952.3 & 0.410 & 3.03 & 64845.4 & -0.103 \\
\hline 3.30 & 50176.2 & -0.065 & 3.12 & 52809.6 & 0.396 & 3.06 & 64858.6 & -0.090 \\
\hline 3.33 & 50199.6 & -0.059 & 3.15 & 52673.8 & 0.383 & 3.09 & 64869.5 & -0.079 \\
\hline 3.36 & 50221.0 & -0.054 & 3.18 & 52544.5 & 0.370 & 3.12 & 64878.5 & -0.069 \\
\hline
\end{tabular}




\begin{tabular}{|c|c|c|c|c|c|c|c|c|}
\hline 3.39 & 50240.6 & -0.049 & 3.21 & 52421.7 & 0.357 & 3.15 & 64885.8 & -0.061 \\
\hline 3.42 & 50258.4 & -0.045 & 3.24 & 52305.1 & 0.345 & 3.18 & 64891.7 & -0.053 \\
\hline 3.45 & 50274.7 & -0.041 & 3.27 & 52194.4 & 0.333 & 3.21 & 64896.5 & -0.046 \\
\hline 3.48 & 50289.5 & -0.037 & 3.30 & 52089.5 & 0.321 & 3.24 & 64900.1 & -0.040 \\
\hline 3.51 & 50303.1 & -0.034 & 3.33 & 51989.9 & 0.309 & 3.27 & 64902.9 & -0.035 \\
\hline 3.54 & 50315.5 & -0.031 & 3.36 & 51895.5 & 0.298 & 3.30 & 64905.0 & -0.030 \\
\hline 3.57 & 50327.0 & -0.028 & 3.39 & 51806.0 & 0.286 & 3.33 & 64905.8 & -0.026 \\
\hline 3.60 & 50337.4 & -0.025 & 3.42 & 51721.2 & 0.276 & 3.36 & 64906.8 & -0.022 \\
\hline 3.63 & 50347.0 & -0.022 & 3.45 & 51640.8 & 0.265 & 3.39 & 64907.4 & -0.019 \\
\hline 3.66 & 50355.8 & -0.020 & 3.48 & 51564.7 & 0.255 & 3.42 & 64907.6 & -0.016 \\
\hline 3.69 & 50363.9 & -0.017 & 3.51 & 51492.6 & 0.245 & 3.45 & 64907.6 & -0.014 \\
\hline 3.72 & 50371.4 & -0.015 & 3.54 & 51424.4 & 0.235 & 3.48 & 64907.4 & -0.011 \\
\hline 3.75 & 50378.4 & -0.013 & 3.57 & 51359.8 & 0.225 & 3.51 & 64906.9 & -0.009 \\
\hline 3.78 & 50384.9 & -0.010 & 3.60 & 51298.7 & 0.216 & 3.54 & 64906.4 & -0.008 \\
\hline 3.81 & 50397.6 & -0.008 & 3.63 & 51240.8 & 0.207 & 3.57 & 64905.8 & -0.006 \\
\hline 3.84 & 50403.5 & -0.006 & 3.66 & 51186.0 & 0.198 & 3.60 & 64905.0 & -0.005 \\
\hline 3.87 & 50409.1 & -0.003 & 3.69 & 51134.2 & 0.190 & 3.63 & 64904.2 & -0.003 \\
\hline 3.90 & 50414.6 & 0.000 & 3.72 & 51085.1 & 0.181 & 3.66 & 64903.5 & -0.002 \\
\hline 3.93 & 50420.1 & 0.003 & 3.75 & 51038.7 & 0.173 & 3.69 & 64902.6 & -0.001 \\
\hline 3.96 & 50425.5 & 0.006 & 3.78 & 50994.5 & 0.165 & 3.72 & 64901.8 & 0.000 \\
\hline 3.99 & 50434.3 & 0.010 & 3.81 & 50954.0 & 0.156 & 3.75 & 64901.0 & 0.001 \\
\hline 4.02 & 50440.3 & 0.014 & 3.84 & 50914.4 & 0.148 & 3.78 & 64900.3 & 0.002 \\
\hline 4.05 & 50446.6 & 0.019 & 3.87 & 50876.7 & 0.140 & 3.81 & 64905.8 & 0.003 \\
\hline 4.08 & 50453.2 & 0.024 & 3.90 & 50842.0 & 0.132 & 3.84 & 64904.7 & 0.003 \\
\hline 4.11 & 50460.0 & 0.029 & 3.93 & 50807.8 & 0.124 & 3.87 & 64903.8 & 0.004 \\
\hline 4.14 & 50466.8 & 0.035 & 3.96 & 50774.8 & 0.115 & 3.90 & 64903.5 & 0.005 \\
\hline 4.17 & 50473.2 & 0.040 & 3.99 & 50743.9 & 0.106 & 3.93 & 64911.1 & 0.006 \\
\hline 4.20 & 50478.7 & 0.045 & 4.02 & 50712.9 & 0.097 & 3.96 & 64910.5 & 0.007 \\
\hline 4.23 & 50483.0 & 0.049 & 4.05 & 50682.8 & 0.088 & 3.99 & 64914.6 & 0.008 \\
\hline 4.26 & 50485.8 & 0.052 & 4.08 & 50653.2 & 0.079 & 4.02 & 64913.9 & 0.009 \\
\hline 4.29 & 50486.6 & 0.055 & 4.11 & 50624.4 & 0.069 & 4.05 & 64913.5 & 0.010 \\
\hline 4.32 & 50472.3 & 0.056 & 4.14 & 50596.6 & 0.059 & 4.08 & 64913.2 & 0.011 \\
\hline 4.35 & 50460.4 & 0.057 & 4.17 & 50570.1 & 0.050 & 4.11 & 64913.2 & 0.012 \\
\hline 4.38 & 50450.6 & 0.057 & 4.20 & 50545.6 & 0.041 & 4.14 & 64913.6 & 0.014 \\
\hline 4.41 & 50442.5 & 0.056 & 4.23 & 50523.1 & 0.033 & 4.17 & 64914.3 & 0.015 \\
\hline 4.44 & 50435.9 & 0.055 & 4.26 & 50503.5 & 0.026 & 4.20 & 64915.2 & 0.017 \\
\hline 4.47 & 50430.5 & 0.054 & 4.32 & 50486.7 & 0.015 & 4.23 & 64916.9 & 0.019 \\
\hline 4.50 & 50426.0 & 0.053 & 4.35 & 50485.2 & 0.011 & 4.26 & 64919.0 & 0.021 \\
\hline 4.53 & 50422.3 & 0.051 & 4.38 & 50482.7 & 0.008 & 4.32 & 64925.4 & 0.026 \\
\hline 4.56 & 50419.3 & 0.049 & 4.41 & 50479.4 & 0.006 & 4.35 & 64929.4 & 0.029 \\
\hline 4.59 & 50416.7 & 0.048 & 4.44 & 50475.6 & 0.004 & 4.38 & 64934.5 & 0.033 \\
\hline 4.62 & 50414.6 & 0.046 & 4.47 & 50471.4 & 0.002 & 4.41 & 64941.2 & 0.038 \\
\hline 4.65 & 50412.8 & 0.044 & 4.50 & 50467.1 & 0.001 & 4.44 & 64948.0 & 0.043 \\
\hline 4.68 & 50411.3 & 0.042 & 4.53 & 50462.7 & 0.000 & 4.47 & 64956.4 & 0.048 \\
\hline
\end{tabular}




\begin{tabular}{|c|c|c|c|c|c|c|c|c|}
\hline 4.71 & 50410.1 & 0.041 & 4.56 & 50458.3 & -0.001 & 4.50 & 64965.7 & 0.054 \\
\hline 4.74 & 50409.0 & 0.039 & 4.59 & 50453.9 & -0.001 & 4.53 & 64967.0 & 0.060 \\
\hline 4.77 & 50408.2 & 0.037 & 4.62 & 50449.7 & -0.002 & 4.56 & 64937.8 & 0.066 \\
\hline \multirow[t]{8}{*}{4.80} & 50407.5 & 0.035 & 4.65 & 50445.7 & -0.002 & 4.59 & 64909.1 & 0.071 \\
\hline & & & 4.68 & 50441.8 & -0.002 & 4.62 & 64881.7 & 0.077 \\
\hline & & & 4.71 & 50438.1 & -0.003 & 4.65 & 64856.1 & 0.081 \\
\hline & & & 4.74 & 50434.7 & -0.003 & 4.68 & 64832.7 & 0.085 \\
\hline & & & 4.77 & 50431.5 & -0.003 & 4.71 & 64811.3 & 0.087 \\
\hline & & & 4.80 & 50428.4 & -0.003 & 4.74 & 64794.0 & 0.090 \\
\hline & & & & & & 4.77 & 64777.9 & 0.091 \\
\hline & & & & & & 4.80 & 64763.9 & 0.092 \\
\hline
\end{tabular}

\begin{tabular}{|c|c|c|c|c|c|c|c|c|}
\hline \multicolumn{3}{|c|}{$(5)^{2} \Pi$} & \multicolumn{3}{|c|}{$(1)^{2} \Delta$} & \multicolumn{3}{|c|}{$(2)^{2} \Delta$} \\
\hline $\mathrm{R}(\AA)$ & $\mathrm{E}\left(\mathrm{cm}^{-1}\right)$ & $\mu$ (a.u.) & $\mathrm{R}(\AA)$ & $\mathrm{E}\left(\mathrm{cm}^{-1}\right)$ & $\mu$ (a.u.) & $\mathrm{R}(\AA)$ & $\mathrm{E}\left(\mathrm{cm}^{-1}\right)$ & $\mu$ (a.u.) \\
\hline 1.41 & 124149.6 & 0.275 & 1.41 & 109361.7 & 1.103 & 1.41 & 119268.0 & 1.125 \\
\hline 1.44 & 117123.0 & 0.319 & 1.44 & 102154.7 & 1.100 & 1.44 & 112217.9 & 1.128 \\
\hline 1.47 & 110979.8 & 0.347 & 1.47 & 95853.7 & 1.093 & 1.47 & 106057.2 & 1.126 \\
\hline 1.50 & 105594.0 & 0.372 & 1.50 & 90325.5 & 1.086 & 1.50 & 100626.8 & 1.123 \\
\hline 1.53 & 100929.2 & 0.389 & 1.53 & 85509.1 & 1.073 & 1.53 & 95935.0 & 1.114 \\
\hline 1.56 & 96858.0 & 0.402 & 1.56 & 81291.6 & 1.058 & 1.56 & 91837.9 & 1.102 \\
\hline 1.59 & 93301.6 & 0.416 & 1.59 & 77593.1 & 1.041 & 1.59 & 88253.6 & 1.089 \\
\hline 1.62 & 90191.7 & 0.428 & 1.62 & 74346.3 & 1.024 & 1.62 & 85114.9 & 1.074 \\
\hline 1.64 & 87762.9 & 0.437 & 1.65 & 71493.0 & 1.005 & 1.65 & 82363.8 & 1.057 \\
\hline 1.66 & 86490.0 & 0.443 & 1.68 & 68982.9 & 0.986 & 1.68 & 79952.3 & 1.040 \\
\hline 1.68 & 85085.2 & 0.449 & 1.71 & 66772.7 & 0.966 & 1.71 & 77836.6 & 1.022 \\
\hline 1.71 & 82995.1 & 0.456 & 1.74 & 64824.9 & 0.946 & 1.74 & 75981.2 & 1.003 \\
\hline 1.74 & 81061.2 & 0.466 & 1.77 & 63107.0 & 0.925 & 1.77 & 74352.0 & 0.984 \\
\hline 1.77 & 79373.8 & 0.473 & 1.80 & 61591.0 & 0.903 & 1.80 & 72922.5 & 0.964 \\
\hline 1.80 & 78143.2 & 0.479 & 1.83 & 60252.4 & 0.882 & 1.83 & 71668.6 & 0.943 \\
\hline 1.83 & 76723.8 & 0.486 & 1.86 & 59069.9 & 0.860 & 1.86 & 70569.4 & 0.922 \\
\hline 1.86 & 75814.8 & 0.492 & 1.89 & 58024.7 & 0.837 & 1.89 & 69606.5 & 0.901 \\
\hline 1.89 & 74807.5 & 0.499 & 1.92 & 57100.7 & 0.815 & 1.92 & 68763.9 & 0.879 \\
\hline 1.92 & 74015.0 & 0.504 & 1.95 & 56283.3 & 0.792 & 1.95 & 68027.3 & 0.856 \\
\hline 1.95 & 73292.4 & 0.510 & 1.98 & 55559.9 & 0.769 & 1.98 & 67384.4 & 0.834 \\
\hline 1.98 & 72751.5 & 0.517 & 2.01 & 54919.4 & 0.746 & 2.01 & 66823.9 & 0.811 \\
\hline 2.01 & 72042.8 & 0.522 & 2.04 & 54351.9 & 0.723 & 2.04 & 66336.4 & 0.788 \\
\hline 2.04 & 71530.6 & 0.526 & 2.07 & 53848.7 & 0.701 & 2.07 & 65913.0 & 0.765 \\
\hline 2.05 & 71380.7 & 0.528 & 2.10 & 53402.1 & 0.678 & 2.10 & 65545.5 & 0.742 \\
\hline 2.06 & 71236.0 & 0.530 & 2.13 & 53005.5 & 0.655 & 2.13 & 65230.7 & 0.718 \\
\hline 2.07 & 71096.5 & 0.532 & 2.16 & 52652.7 & 0.633 & 2.16 & 64957.4 & 0.695 \\
\hline 2.08 & 70962.1 & 0.533 & 2.19 & 52338.9 & 0.611 & 2.19 & 64723.3 & 0.673 \\
\hline 2.09 & 70832.6 & 0.535 & 2.22 & 52059.0 & 0.589 & 2.22 & 64523.1 & 0.650 \\
\hline 2.10 & 70707.8 & 0.536 & 2.25 & 51809.1 & 0.567 & 2.25 & 64351.8 & 0.627 \\
\hline 2.11 & 70588.2 & 0.538 & 2.28 & 51585.6 & 0.546 & 2.28 & 64205.3 & 0.605 \\
\hline
\end{tabular}




\begin{tabular}{|c|c|c|c|c|c|c|c|c|}
\hline 2.12 & 70472.3 & 0.539 & 2.31 & 51385.7 & 0.525 & 2.31 & 64082.8 & 0.583 \\
\hline 2.13 & 70360.8 & 0.540 & 2.34 & 51206.5 & 0.505 & 2.34 & 63980.0 & 0.561 \\
\hline 2.14 & 70253.5 & 0.541 & 2.37 & 51045.7 & 0.485 & 2.37 & 63894.5 & 0.540 \\
\hline 2.16 & 69870.8 & 0.543 & 2.40 & 50901.2 & 0.466 & 2.40 & 63824.5 & 0.519 \\
\hline 2.19 & 69668.9 & 0.544 & 2.43 & 50771.0 & 0.447 & 2.43 & 63768.7 & 0.499 \\
\hline 2.22 & 69438.7 & 0.545 & 2.46 & 50653.2 & 0.429 & 2.46 & 63725.4 & 0.479 \\
\hline 2.25 & 69220.9 & 0.546 & 2.49 & 50546.4 & 0.411 & 2.49 & 63694.9 & 0.460 \\
\hline 2.28 & 69019.6 & 0.546 & 2.52 & 50449.9 & 0.394 & 2.52 & 63673.0 & 0.441 \\
\hline 2.31 & 68834.4 & 0.548 & 2.55 & 50363.4 & 0.378 & 2.55 & 63654.1 & 0.423 \\
\hline 2.34 & 68665.0 & 0.548 & 2.58 & 50286.5 & 0.362 & 2.58 & 63637.6 & 0.405 \\
\hline 2.37 & 68508.2 & 0.548 & 2.61 & 50218.2 & 0.346 & 2.61 & 63623.1 & 0.388 \\
\hline 2.40 & 68364.5 & 0.547 & 2.64 & 50157.6 & 0.331 & 2.64 & 63611.5 & 0.372 \\
\hline 2.43 & 68231.5 & 0.546 & 2.67 & 50103.7 & 0.317 & 2.67 & 63601.6 & 0.356 \\
\hline 2.46 & 68109.1 & 0.543 & 2.70 & 50055.8 & 0.303 & 2.70 & 63593.5 & 0.340 \\
\hline 2.49 & 67994.7 & 0.541 & 2.73 & 50013.5 & 0.290 & 2.73 & 63588.0 & 0.325 \\
\hline 2.52 & 67888.7 & 0.538 & 2.76 & 49976.2 & 0.277 & 2.76 & 63584.5 & 0.311 \\
\hline 2.55 & 67789.4 & 0.534 & 2.79 & 49943.4 & 0.265 & 2.79 & 63582.7 & 0.297 \\
\hline 2.58 & 67696.4 & 0.531 & 2.82 & 49915.0 & 0.253 & 2.82 & 63582.4 & 0.284 \\
\hline 2.61 & 67610.5 & 0.527 & 2.85 & 49890.1 & 0.242 & 2.85 & 63583.8 & 0.271 \\
\hline 2.64 & 67527.1 & 0.523 & 2.88 & 49868.5 & 0.231 & 2.88 & 63586.5 & 0.259 \\
\hline 2.67 & 67447.8 & 0.518 & 2.91 & 49850.0 & 0.221 & 2.91 & 63590.4 & 0.247 \\
\hline 2.70 & 67372.2 & 0.514 & 2.94 & 49834.4 & 0.211 & 2.94 & 63595.5 & 0.236 \\
\hline 2.73 & 67299.9 & 0.509 & 2.97 & 49821.2 & 0.202 & 2.97 & 63601.5 & 0.225 \\
\hline 2.76 & 67231.0 & 0.505 & 3.00 & 49810.5 & 0.193 & 3.00 & 63608.6 & 0.215 \\
\hline 2.79 & 67164.3 & 0.500 & 3.03 & 49801.9 & 0.184 & 3.03 & 63616.4 & 0.205 \\
\hline 2.82 & 67099.9 & 0.495 & 3.06 & 49795.3 & 0.176 & 3.06 & 63625.0 & 0.196 \\
\hline 2.85 & 67037.6 & 0.490 & 3.09 & 49790.4 & 0.168 & 3.09 & 63634.3 & 0.187 \\
\hline 2.88 & 66977.5 & 0.485 & 3.12 & 49787.2 & 0.160 & 3.12 & 63644.2 & 0.178 \\
\hline 2.91 & 66919.1 & 0.480 & 3.15 & 49785.4 & 0.153 & 3.15 & 63654.6 & 0.170 \\
\hline 2.94 & 66862.1 & 0.475 & 3.18 & 49785.0 & 0.146 & 3.18 & 63665.6 & 0.162 \\
\hline 2.97 & 66809.0 & 0.468 & 3.21 & 49785.8 & 0.140 & 3.21 & 63677.1 & 0.155 \\
\hline 3.00 & 66754.9 & 0.463 & 3.24 & 49787.7 & 0.134 & 3.24 & 63688.8 & 0.148 \\
\hline 3.03 & 66702.2 & 0.457 & 3.27 & 49790.5 & 0.128 & 3.27 & 63700.8 & 0.141 \\
\hline 3.06 & 66649.2 & 0.452 & 3.30 & 49794.3 & 0.122 & 3.30 & 63713.1 & 0.135 \\
\hline 3.09 & 66599.8 & 0.445 & 3.33 & 49798.8 & 0.117 & 3.33 & 63725.6 & 0.128 \\
\hline 3.12 & 66548.0 & 0.440 & 3.36 & 49804.0 & 0.112 & 3.36 & 63738.2 & 0.123 \\
\hline 3.15 & 66498.9 & 0.433 & 3.39 & 49809.8 & 0.107 & 3.39 & 63750.9 & 0.117 \\
\hline 3.18 & 66451.0 & 0.427 & 3.42 & 49816.1 & 0.102 & 3.42 & 63763.7 & 0.112 \\
\hline 3.21 & 66404.1 & 0.421 & 3.45 & 49822.9 & 0.098 & 3.45 & 63776.5 & 0.107 \\
\hline 3.24 & 66357.9 & 0.414 & 3.48 & 49830.1 & 0.094 & 3.48 & 63789.3 & 0.102 \\
\hline 3.27 & 66312.7 & 0.407 & 3.51 & 49837.6 & 0.090 & 3.51 & 63802.1 & 0.097 \\
\hline 3.30 & 66268.2 & 0.401 & 3.54 & 49845.4 & 0.086 & 3.54 & 63814.9 & 0.093 \\
\hline 3.33 & 66224.5 & 0.394 & 3.57 & 49853.4 & 0.082 & 3.57 & 63827.5 & 0.089 \\
\hline 3.36 & 66181.6 & 0.387 & 3.60 & 49861.7 & 0.079 & 3.60 & 63840.1 & 0.085 \\
\hline
\end{tabular}




\begin{tabular}{|c|c|c|c|c|c|c|c|c|}
\hline 3.39 & 66139.4 & 0.380 & 3.63 & 49870.0 & 0.075 & 3.63 & 63852.5 & 0.081 \\
\hline 3.42 & 66098.0 & 0.373 & 3.66 & 49878.5 & 0.072 & 3.66 & 63864.8 & 0.078 \\
\hline 3.45 & 66057.4 & 0.366 & 3.69 & 49887.1 & 0.069 & 3.69 & 63877.0 & 0.074 \\
\hline 3.48 & 66017.4 & 0.359 & 3.72 & 49895.8 & 0.066 & 3.72 & 63889.0 & 0.071 \\
\hline 3.51 & 65978.4 & 0.352 & 3.75 & 49904.6 & 0.064 & 3.75 & 63901.0 & 0.068 \\
\hline 3.54 & 65939.7 & 0.345 & 3.78 & 49913.4 & 0.061 & 3.78 & 63912.7 & 0.065 \\
\hline 3.57 & 65901.6 & 0.338 & 3.81 & 49922.1 & 0.059 & 3.81 & 63924.3 & 0.062 \\
\hline 3.60 & 65868.4 & 0.331 & 3.84 & 49930.9 & 0.056 & 3.84 & 63935.6 & 0.059 \\
\hline 3.63 & 65838.5 & 0.325 & 3.87 & 49939.7 & 0.054 & 3.87 & 63947.1 & 0.057 \\
\hline 3.66 & 65799.7 & 0.318 & 3.90 & 49948.4 & 0.052 & 3.90 & 63958.1 & 0.054 \\
\hline 3.69 & 65764.1 & 0.311 & 3.93 & 49957.0 & 0.050 & 3.93 & 63968.9 & 0.052 \\
\hline 3.72 & 65730.0 & 0.304 & 3.96 & 49965.6 & 0.048 & 3.96 & 63979.6 & 0.050 \\
\hline 3.75 & 65696.8 & 0.297 & 3.99 & 49974.1 & 0.046 & 3.99 & 63990.3 & 0.048 \\
\hline 3.78 & 65661.3 & 0.290 & 4.02 & 49982.6 & 0.044 & 4.02 & 64000.6 & 0.046 \\
\hline 3.81 & 65629.0 & 0.283 & 4.05 & 49990.9 & 0.042 & 4.05 & 64010.7 & 0.044 \\
\hline 3.84 & 65596.6 & 0.276 & 4.08 & 49999.2 & 0.040 & 4.08 & 64020.6 & 0.042 \\
\hline 3.87 & 65565.0 & 0.269 & 4.11 & 50007.4 & 0.039 & 4.11 & 64030.4 & 0.040 \\
\hline 3.90 & 65534.1 & 0.262 & 4.14 & 50015.5 & 0.037 & 4.14 & 64039.9 & 0.039 \\
\hline 3.93 & 65504.0 & 0.255 & 4.17 & 50023.5 & 0.036 & 4.17 & 64049.3 & 0.037 \\
\hline 3.96 & 65474.3 & 0.248 & 4.20 & 50031.4 & 0.034 & 4.20 & 64058.3 & 0.035 \\
\hline 3.99 & 65445.7 & 0.241 & 4.23 & 50039.2 & 0.033 & 4.23 & 64067.3 & 0.034 \\
\hline 4.02 & 65417.2 & 0.233 & 4.26 & 50046.9 & 0.032 & 4.26 & 64076.1 & 0.033 \\
\hline 4.05 & 65386.5 & 0.225 & 4.29 & 50054.5 & 0.030 & 4.29 & 64085.8 & 0.031 \\
\hline 4.08 & 65362.0 & 0.219 & 4.32 & 50061.9 & 0.029 & 4.32 & 64094.3 & 0.030 \\
\hline 4.11 & 65335.2 & 0.212 & 4.35 & 50069.3 & 0.028 & 4.35 & 64103.3 & 0.029 \\
\hline 4.14 & 65309.0 & 0.204 & 4.38 & 50076.5 & 0.027 & 4.38 & 64111.4 & 0.028 \\
\hline 4.17 & 65283.1 & 0.197 & 4.41 & 50083.5 & 0.026 & 4.41 & 64119.3 & 0.026 \\
\hline 4.20 & 65259.3 & 0.190 & 4.44 & 50090.4 & 0.025 & 4.44 & 64127.0 & 0.025 \\
\hline 4.23 & 65232.9 & 0.182 & 4.47 & 50097.1 & 0.024 & 4.47 & 64134.2 & 0.024 \\
\hline 4.26 & 65207.8 & 0.174 & 4.50 & 50103.7 & 0.023 & 4.50 & 64141.6 & 0.023 \\
\hline 4.29 & 65181.4 & 0.165 & 4.53 & 50110.2 & 0.022 & 4.53 & 64148.7 & 0.022 \\
\hline 4.32 & 65156.2 & 0.156 & 4.56 & 50116.5 & 0.021 & 4.56 & 64155.7 & 0.021 \\
\hline 4.35 & 65132.6 & 0.148 & 4.59 & 50122.8 & 0.020 & 4.59 & 64163.3 & 0.021 \\
\hline 4.38 & 65107.4 & 0.140 & 4.62 & 50129.1 & 0.020 & 4.62 & 64169.9 & 0.020 \\
\hline 4.41 & 65078.2 & 0.129 & 4.65 & 50135.0 & 0.019 & 4.65 & 64176.3 & 0.019 \\
\hline 4.44 & 65053.4 & 0.120 & 4.68 & 50140.7 & 0.018 & 4.68 & 64182.5 & 0.018 \\
\hline 4.47 & 65025.2 & 0.110 & 4.71 & 50146.2 & 0.017 & 4.71 & 64188.6 & 0.017 \\
\hline 4.50 & 64996.3 & 0.099 & 4.74 & 50151.6 & 0.017 & 4.74 & 64194.5 & 0.017 \\
\hline 4.53 & 64975.7 & 0.089 & 4.77 & 50156.8 & 0.016 & 4.77 & 64201.2 & 0.016 \\
\hline 4.56 & 64986.0 & 0.078 & 4.80 & 50161.9 & 0.015 & 4.80 & 64206.7 & 0.015 \\
\hline 4.59 & 64996.2 & 0.068 & & & & & & \\
\hline 4.62 & 65005.6 & 0.059 & & & & & & \\
\hline 4.65 & 65013.9 & 0.050 & & & & & & \\
\hline 4.68 & 65020.8 & 0.042 & & & & & & \\
\hline
\end{tabular}




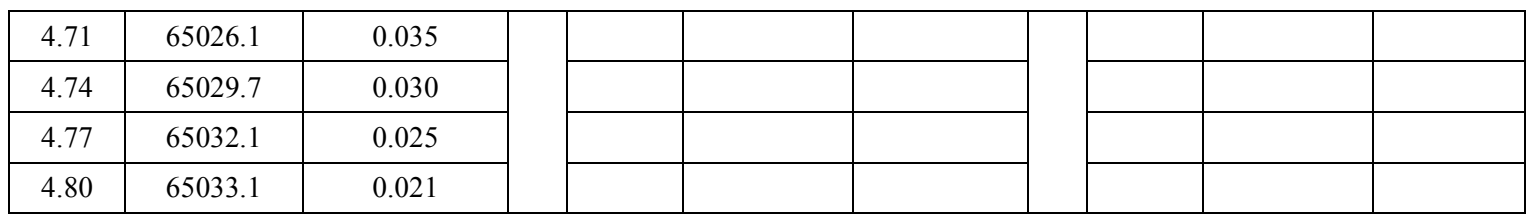

\begin{tabular}{|c|c|c|c|c|c|c|c|c|}
\hline \multicolumn{3}{|c|}{$(1)^{2} \Sigma^{-}$} & \multicolumn{3}{|c|}{$(2)^{2} \Sigma^{-}$} & \multicolumn{3}{|c|}{$(1)^{4} \Sigma^{+}$} \\
\hline $\mathrm{R}(\AA)$ & $\mathrm{E}\left(\mathrm{cm}^{-1}\right)$ & $\mu$ (a.u.) & $\mathrm{R}(\AA)$ & $\mathrm{E}\left(\mathrm{cm}^{-1}\right)$ & $\mu$ (a.u.) & $\mathrm{R}(\AA)$ & $\mathrm{E}\left(\mathrm{cm}^{-1}\right)$ & $\mu$ (a.u.) \\
\hline 1.41 & 110050.6 & 1.117 & 1.41 & 119956.7 & 1.136 & 1.41 & 105651.1 & 1.044 \\
\hline 1.44 & 102832.2 & 1.114 & 1.44 & 112875.5 & 1.138 & 1.44 & 98542.4 & 1.032 \\
\hline 1.47 & 96519.1 & 1.107 & 1.47 & 106685.8 & 1.136 & 1.47 & 92340.2 & 1.018 \\
\hline 1.50 & 90977.6 & 1.099 & 1.50 & 101223.0 & 1.133 & 1.50 & 86926.4 & 1.003 \\
\hline 1.53 & 86148.4 & 1.086 & 1.53 & 96508.5 & 1.123 & 1.53 & 82198.0 & 0.987 \\
\hline 1.56 & 81917.9 & 1.071 & 1.56 & 92391.2 & 1.111 & 1.56 & 78065.8 & 0.970 \\
\hline 1.59 & 78206.4 & 1.054 & 1.59 & 88788.4 & 1.097 & 1.59 & 74452.1 & 0.953 \\
\hline 1.62 & 74946.4 & 1.037 & 1.62 & 85632.9 & 1.082 & 1.62 & 71289.9 & 0.934 \\
\hline 1.65 & 72079.9 & 1.018 & 1.65 & 82866.9 & 1.066 & 1.65 & 68521.0 & 0.916 \\
\hline 1.68 & 69556.6 & 0.998 & 1.68 & 80440.9 & 1.048 & 1.68 & 66095.2 & 0.897 \\
\hline 1.71 & 67333.3 & 0.978 & 1.71 & 78311.9 & 1.030 & 1.71 & 63969.3 & 0.878 \\
\hline 1.74 & 65372.6 & 0.957 & 1.74 & 76444.4 & 1.011 & 1.74 & 62105.6 & 0.859 \\
\hline 1.77 & 63641.8 & 0.936 & 1.77 & 74803.7 & 0.991 & 1.77 & 60471.6 & 0.839 \\
\hline 1.80 & 62113.0 & 0.914 & 1.80 & 73363.3 & 0.971 & 1.80 & 59039.1 & 0.819 \\
\hline 1.83 & 60761.8 & 0.892 & 1.83 & 72099.3 & 0.950 & 1.83 & 57783.5 & 0.799 \\
\hline 1.86 & 59566.8 & 0.870 & 1.86 & 70990.4 & 0.929 & 1.86 & 56683.5 & 0.779 \\
\hline 1.89 & 58509.3 & 0.847 & 1.89 & 70018.3 & 0.907 & 1.89 & 55720.1 & 0.759 \\
\hline 1.92 & 57573.0 & 0.825 & 1.92 & 69166.9 & 0.885 & 1.92 & 54877.0 & 0.738 \\
\hline 1.95 & 56743.7 & 0.802 & 1.95 & 68421.9 & 0.863 & 1.95 & 54139.6 & 0.718 \\
\hline 1.98 & 56008.5 & 0.779 & 1.98 & 67770.8 & 0.840 & 1.98 & 53495.4 & 0.697 \\
\hline 2.01 & 55356.3 & 0.756 & 2.01 & 67202.5 & 0.817 & 2.01 & 52933.0 & 0.677 \\
\hline 2.04 & 54777.3 & 0.733 & 2.04 & 66707.3 & 0.794 & 2.04 & 52442.7 & 0.656 \\
\hline 2.07 & 54262.8 & 0.709 & 2.07 & 66276.4 & 0.771 & 2.07 & 52015.4 & 0.636 \\
\hline 2.10 & 53805.1 & 0.687 & 2.10 & 65901.7 & 0.747 & 2.10 & 51643.7 & 0.616 \\
\hline 2.13 & 53397.5 & 0.664 & 2.13 & 65577.7 & 0.724 & 2.13 & 51320.6 & 0.596 \\
\hline 2.16 & 53034.0 & 0.641 & 2.16 & 65297.5 & 0.701 & 2.16 & 51040.2 & 0.576 \\
\hline 2.19 & 52709.6 & 0.619 & 2.19 & 65056.5 & 0.678 & 2.19 & 50797.0 & 0.556 \\
\hline 2.22 & 52419.2 & 0.597 & 2.22 & 64851.9 & 0.655 & 2.22 & 50586.6 & 0.536 \\
\hline 2.25 & 52159.1 & 0.575 & 2.25 & 64674.0 & 0.632 & 2.25 & 50404.8 & 0.517 \\
\hline 2.28 & 51925.8 & 0.554 & 2.28 & 64519.8 & 0.610 & 2.28 & 50248.0 & 0.498 \\
\hline 2.31 & 51715.8 & 0.533 & 2.31 & 64391.5 & 0.588 & 2.31 & 50113.0 & 0.480 \\
\hline 2.34 & 51527.1 & 0.512 & 2.34 & 64282.2 & 0.566 & 2.34 & 49997.2 & 0.462 \\
\hline 2.37 & 51356.8 & 0.492 & 2.37 & 64190.2 & 0.545 & 2.37 & 49898.3 & 0.444 \\
\hline 2.40 & 51203.0 & 0.473 & 2.40 & 64113.9 & 0.524 & 2.40 & 49814.0 & 0.427 \\
\hline 2.43 & 51063.7 & 0.454 & 2.43 & 64051.8 & 0.503 & 2.43 & 49742.5 & 0.410 \\
\hline 2.46 & 50937.1 & 0.435 & 2.46 & 64002.1 & 0.483 & 2.46 & 49682.4 & 0.394 \\
\hline 2.49 & 50821.6 & 0.417 & 2.49 & 63965.4 & 0.464 & 2.49 & 49632.1 & 0.378 \\
\hline
\end{tabular}




\begin{tabular}{|c|c|c|c|c|c|c|c|c|}
\hline 2.52 & 50716.6 & 0.400 & 2.52 & 63936.6 & 0.445 & 2.52 & 49590.5 & 0.363 \\
\hline 2.55 & 50621.8 & 0.383 & 2.55 & 63912.3 & 0.426 & 2.55 & 49556.4 & 0.348 \\
\hline 2.58 & 50536.8 & 0.367 & 2.58 & 63889.8 & 0.409 & 2.58 & 49529.1 & 0.333 \\
\hline 2.61 & 50460.6 & 0.352 & 2.61 & 63869.3 & 0.391 & 2.61 & 49507.5 & 0.320 \\
\hline 2.64 & 50392.3 & 0.336 & 2.64 & 63851.6 & 0.375 & 2.64 & 49491.1 & 0.306 \\
\hline 2.67 & 50331.0 & 0.322 & 2.67 & 63835.9 & 0.359 & 2.67 & 49479.1 & 0.293 \\
\hline 2.70 & 50275.8 & 0.308 & 2.70 & 63822.1 & 0.343 & 2.70 & 49471.1 & 0.281 \\
\hline 2.73 & 50226.4 & 0.295 & 2.73 & 63811.0 & 0.328 & 2.73 & 49466.6 & 0.269 \\
\hline 2.76 & 50182.2 & 0.282 & 2.76 & 63801.9 & 0.314 & 2.76 & 49465.0 & 0.257 \\
\hline 2.79 & 50142.7 & 0.269 & 2.79 & 63794.7 & 0.300 & 2.79 & 49466.1 & 0.246 \\
\hline 2.82 & 50107.7 & 0.257 & 2.82 & 63789.1 & 0.286 & 2.82 & 49469.6 & 0.235 \\
\hline 2.85 & 50076.5 & 0.246 & 2.85 & 63785.3 & 0.274 & 2.85 & 49475.0 & 0.225 \\
\hline 2.88 & 50048.8 & 0.235 & 2.88 & 63782.9 & 0.261 & 2.88 & 49485.4 & 0.215 \\
\hline 2.91 & 50024.3 & 0.225 & 2.91 & 63781.8 & 0.250 & 2.91 & 49496.6 & 0.206 \\
\hline 2.94 & 50002.9 & 0.215 & 2.94 & 63782.0 & 0.238 & 2.94 & 49508.3 & 0.197 \\
\hline 2.97 & 49984.2 & 0.205 & 2.97 & 63783.4 & 0.227 & 2.97 & 49519.6 & 0.188 \\
\hline 3.00 & 49968.0 & 0.196 & 3.00 & 63785.8 & 0.217 & 3.00 & 49531.9 & 0.180 \\
\hline 3.03 & 49954.1 & 0.187 & 3.03 & 63789.1 & 0.207 & 3.03 & 49545.2 & 0.172 \\
\hline 3.06 & 49942.4 & 0.179 & 3.06 & 63793.4 & 0.198 & 3.06 & 49559.3 & 0.164 \\
\hline 3.09 & 49932.6 & 0.171 & 3.09 & 63798.4 & 0.189 & 3.09 & 49574.0 & 0.157 \\
\hline 3.12 & 49924.5 & 0.163 & 3.12 & 63804.1 & 0.180 & 3.12 & 49589.3 & 0.150 \\
\hline 3.15 & 49918.1 & 0.156 & 3.15 & 63810.5 & 0.172 & 3.15 & 49605.0 & 0.144 \\
\hline 3.18 & 49913.2 & 0.149 & 3.18 & 63817.5 & 0.164 & 3.18 & 49621.1 & 0.137 \\
\hline 3.21 & 49909.7 & 0.142 & 3.21 & 63825.2 & 0.156 & 3.21 & 49637.6 & 0.131 \\
\hline 3.24 & 49907.4 & 0.136 & 3.24 & 63833.2 & 0.149 & 3.24 & 49654.3 & 0.126 \\
\hline 3.27 & 49906.2 & 0.130 & 3.27 & 63841.6 & 0.142 & 3.27 & 49671.2 & 0.120 \\
\hline 3.30 & 49906.0 & 0.124 & 3.30 & 63850.3 & 0.136 & 3.30 & 49688.3 & 0.115 \\
\hline 3.33 & 49906.8 & 0.119 & 3.33 & 63859.4 & 0.130 & 3.33 & 49705.4 & 0.110 \\
\hline 3.36 & 49908.3 & 0.114 & 3.36 & 63868.6 & 0.124 & 3.36 & 49722.5 & 0.105 \\
\hline 3.39 & 49910.6 & 0.109 & 3.39 & 63878.1 & 0.118 & 3.39 & 49739.6 & 0.101 \\
\hline 3.42 & 49913.5 & 0.104 & 3.42 & 63887.7 & 0.113 & 3.42 & 49756.7 & 0.096 \\
\hline 3.45 & 49917.0 & 0.100 & 3.45 & 63897.4 & 0.108 & 3.45 & 49773.6 & 0.092 \\
\hline 3.48 & 49921.0 & 0.095 & 3.48 & 63907.2 & 0.103 & 3.48 & 49790.4 & 0.088 \\
\hline 3.51 & 49925.4 & 0.091 & 3.51 & 63917.1 & 0.098 & 3.51 & 49807.0 & 0.085 \\
\hline 3.54 & 49930.3 & 0.087 & 3.54 & 63927.0 & 0.094 & 3.54 & 49823.4 & 0.081 \\
\hline 3.57 & 49935.4 & 0.084 & 3.57 & 63936.9 & 0.090 & 3.57 & 49839.9 & 0.078 \\
\hline 3.60 & 49940.9 & 0.080 & 3.60 & 63946.8 & 0.086 & 3.60 & 49855.9 & 0.074 \\
\hline 3.63 & 49946.6 & 0.077 & 3.63 & 63956.6 & 0.082 & 3.63 & 49871.6 & 0.071 \\
\hline 3.66 & 49952.6 & 0.074 & 3.66 & 63966.4 & 0.078 & 3.66 & 49887.1 & 0.068 \\
\hline 3.69 & 49958.7 & 0.071 & 3.69 & 63976.1 & 0.075 & 3.69 & 49902.3 & 0.065 \\
\hline 3.72 & 49965.0 & 0.068 & 3.72 & 63985.7 & 0.072 & 3.72 & 49917.3 & 0.063 \\
\hline 3.75 & 49971.5 & 0.065 & 3.75 & 63995.3 & 0.069 & 3.75 & 49932.0 & 0.060 \\
\hline 3.78 & 49978.0 & 0.062 & 3.78 & 64004.7 & 0.066 & 3.78 & 49946.4 & 0.058 \\
\hline 3.81 & 49984.6 & 0.060 & 3.81 & 64014.1 & 0.063 & 3.81 & 49960.5 & 0.055 \\
\hline
\end{tabular}




\begin{tabular}{|c|c|c|c|c|c|c|c|c|}
\hline 3.84 & 49991.2 & 0.057 & 3.84 & 64023.3 & 0.060 & 3.84 & 49974.4 & 0.053 \\
\hline 3.87 & 49998.1 & 0.055 & 3.87 & 64032.5 & 0.058 & 3.87 & 49988.0 & 0.051 \\
\hline 3.90 & 50004.9 & 0.053 & 3.90 & 64041.5 & 0.055 & 3.90 & 50001.3 & 0.049 \\
\hline 3.93 & 50011.7 & 0.051 & 3.93 & 64050.3 & 0.053 & 3.93 & 50014.3 & 0.047 \\
\hline 3.96 & 50018.4 & 0.048 & 3.96 & 64059.0 & 0.051 & 3.96 & 50027.9 & 0.045 \\
\hline 3.99 & 50025.3 & 0.047 & 3.99 & 64067.9 & 0.048 & 3.99 & 50040.4 & 0.043 \\
\hline 4.02 & 50032.0 & 0.045 & 4.02 & 64076.4 & 0.046 & 4.00 & 50044.5 & 0.042 \\
\hline 4.05 & 50038.8 & 0.043 & 4.05 & 64084.7 & 0.044 & 4.03 & 50056.6 & 0.041 \\
\hline 4.08 & 50045.5 & 0.041 & 4.08 & 64092.9 & 0.043 & 4.06 & 50068.6 & 0.039 \\
\hline 4.11 & 50052.2 & 0.040 & 4.11 & 64100.9 & 0.041 & 4.09 & 50080.2 & 0.037 \\
\hline 4.14 & 50058.9 & 0.038 & 4.14 & 64108.8 & 0.039 & 4.12 & 50091.6 & 0.036 \\
\hline 4.17 & 50065.5 & 0.036 & 4.17 & 64116.6 & 0.037 & 4.15 & 50102.8 & 0.034 \\
\hline 4.20 & 50072.0 & 0.035 & 4.20 & 64124.0 & 0.036 & 4.18 & 50113.7 & 0.033 \\
\hline 4.23 & 50078.5 & 0.034 & 4.23 & 64131.5 & 0.034 & 4.21 & 50124.4 & 0.032 \\
\hline 4.26 & 50085.0 & 0.032 & 4.26 & 64138.9 & 0.033 & 4.24 & 50134.8 & 0.030 \\
\hline 4.29 & 50091.4 & 0.031 & 4.29 & 64146.6 & 0.032 & 4.27 & 50145.0 & 0.029 \\
\hline 4.32 & 50097.6 & 0.030 & 4.32 & 64153.8 & 0.030 & 4.30 & 50155.0 & 0.028 \\
\hline 4.35 & 50103.9 & 0.029 & 4.35 & 64161.1 & 0.029 & 4.33 & 50164.8 & 0.027 \\
\hline 4.38 & 50109.9 & 0.027 & 4.38 & 64167.9 & 0.028 & 4.36 & 50174.3 & 0.026 \\
\hline 4.41 & 50116.0 & 0.026 & 4.41 & 64174.5 & 0.027 & 4.39 & 50183.6 & 0.025 \\
\hline 4.44 & 50121.8 & 0.025 & 4.44 & 64181.0 & 0.026 & 4.42 & 50192.6 & 0.024 \\
\hline 4.47 & 50127.6 & 0.024 & 4.47 & 64187.0 & 0.025 & 4.45 & 50201.5 & 0.023 \\
\hline 4.50 & 50133.3 & 0.023 & 4.50 & 64193.1 & 0.024 & 4.48 & 50210.1 & 0.022 \\
\hline 4.53 & 50138.8 & 0.022 & 4.53 & 64199.1 & 0.023 & & & \\
\hline 4.56 & 50144.3 & 0.022 & 4.56 & 64204.9 & 0.022 & & & \\
\hline 4.59 & 50149.7 & 0.021 & 4.59 & 64211.5 & 0.021 & & & \\
\hline 4.62 & 50155.2 & 0.020 & 4.62 & 64217.0 & 0.020 & & & \\
\hline 4.65 & 50160.3 & 0.019 & 4.65 & 64222.4 & 0.019 & & & \\
\hline 4.68 & 50165.3 & 0.018 & 4.68 & 64227.6 & 0.018 & & & \\
\hline 4.71 & 50170.1 & 0.018 & 4.71 & 64232.7 & 0.018 & & & \\
\hline 4.74 & 50174.8 & 0.017 & 4.74 & 64237.5 & 0.017 & & & \\
\hline 4.77 & 50179.3 & 0.016 & 4.77 & 64243.4 & 0.016 & & & \\
\hline 4.80 & 50183.8 & 0.016 & 4.80 & 64247.9 & 0.016 & & & \\
\hline
\end{tabular}

\begin{tabular}{|c|c|c|c|c|c|c|c|c|}
\hline \multicolumn{3}{|c|}{$(2)^{4} \Sigma^{+}$} & \multicolumn{3}{|c|}{$(3)^{4} \Sigma^{+}$} & \multicolumn{3}{|c|}{$(4)^{4} \Sigma^{+}$} \\
\hline $\mathrm{R}(\AA)$ & $\mathrm{E}\left(\mathrm{cm}^{-1}\right)$ & $\mu$ (a.u.) & $\mathrm{R}(\AA)$ & $\mathrm{E}\left(\mathrm{cm}^{-1}\right)$ & $\mu$ (a.u.) & $\mathrm{R}(\AA)$ & $\mathrm{E}\left(\mathrm{cm}^{-1}\right)$ & $\mu$ (a.u.) \\
\hline 1.41 & 120935.2 & -0.497 & 1.41 & 131014.7 & 1.659 & 1.41 & 146500.6 & 2.302 \\
\hline 1.44 & 114881.3 & -0.431 & 1.44 & 125028.8 & 1.640 & 1.44 & 140186.9 & 2.242 \\
\hline 1.47 & 109563.7 & -0.369 & 1.47 & 119770.9 & 1.619 & 1.47 & 134542.6 & 2.170 \\
\hline 1.50 & 104881.8 & -0.308 & 1.50 & 115146.4 & 1.593 & 1.50 & 129493.8 & 2.089 \\
\hline 1.53 & 100748.4 & -0.248 & 1.53 & 111072.4 & 1.565 & 1.53 & 124968.4 & 1.997 \\
\hline 1.56 & 97087.4 & -0.188 & 1.56 & 107476.4 & 1.533 & 1.56 & 120951.9 & 1.880 \\
\hline 1.59 & 93833.0 & -0.128 & 1.59 & 104294.6 & 1.498 & 1.59 & 117316.0 & 1.748 \\
\hline 1.62 & 90927.8 & -0.067 & 1.62 & 101469.7 & 1.460 & 1.62 & 114051.7 & 1.592 \\
\hline
\end{tabular}




\begin{tabular}{|c|c|c|c|c|c|c|c|c|}
\hline 1.65 & 88322.4 & -0.006 & 1.68 & 96685.9 & 1.373 & 1.65 & 111117.4 & 1.413 \\
\hline 1.68 & 85974.1 & 0.055 & 1.71 & 94639.8 & 1.325 & 1.68 & 108482.6 & 1.214 \\
\hline 1.71 & 83846.4 & 0.117 & 1.74 & 92808.3 & 1.272 & 1.71 & 106130.8 & 0.981 \\
\hline 1.74 & 81907.8 & 0.178 & 1.77 & 91052.8 & 1.215 & 1.74 & 104130.1 & 0.600 \\
\hline 1.77 & 80131.6 & 0.238 & 1.80 & 89637.8 & 1.161 & 1.77 & 101835.9 & 1.843 \\
\hline 1.80 & 78495.1 & 0.296 & 1.83 & 88308.9 & 1.101 & 1.80 & 100186.3 & 1.396 \\
\hline 1.83 & 76979.4 & 0.353 & 1.86 & 87140.2 & 1.042 & 1.83 & 98684.4 & 1.155 \\
\hline 1.86 & 75568.3 & 0.406 & 1.89 & 86075.1 & 0.981 & 1.86 & 97334.2 & 1.004 \\
\hline 1.89 & 74248.6 & 0.456 & 1.92 & 85100.8 & 0.919 & 1.89 & 96127.3 & 0.891 \\
\hline 1.92 & 73009.3 & 0.502 & 1.95 & 84199.5 & 0.856 & 1.92 & 95051.3 & 0.795 \\
\hline 1.95 & 71841.4 & 0.543 & 1.98 & 83376.6 & 0.795 & 1.95 & 94093.2 & 0.710 \\
\hline 1.98 & 70737.6 & 0.580 & 2.01 & 82619.2 & 0.737 & 1.98 & 93240.6 & 0.632 \\
\hline 2.01 & 69691.8 & 0.611 & 2.04 & 81923.4 & 0.681 & 2.01 & 92482.0 & 0.560 \\
\hline 2.04 & 68699.3 & 0.638 & 2.07 & 81286.0 & 0.628 & 2.04 & 91807.0 & 0.492 \\
\hline 2.07 & 67756.0 & 0.660 & 2.10 & 80702.6 & 0.579 & 2.07 & 91206.5 & 0.432 \\
\hline 2.10 & 66858.5 & 0.678 & 2.13 & 80169.4 & 0.534 & 2.10 & 90671.3 & 0.371 \\
\hline 2.13 & 66004.1 & 0.691 & 2.19 & 79236.9 & 0.455 & 2.13 & 90194.4 & 0.315 \\
\hline 2.16 & 65190.3 & 0.700 & 2.22 & 78830.8 & 0.421 & 2.16 & 89769.1 & 0.261 \\
\hline 2.19 & 64415.2 & 0.706 & 2.25 & 78460.3 & 0.391 & 2.19 & 89390.4 & 0.216 \\
\hline 2.22 & 63676.8 & 0.708 & 2.28 & 78122.3 & 0.364 & 2.22 & 89051.6 & 0.169 \\
\hline 2.25 & 62973.7 & 0.707 & 2.31 & 77814.3 & 0.340 & 2.25 & 88748.9 & 0.125 \\
\hline 2.28 & 62304.1 & 0.704 & 2.34 & 77533.5 & 0.319 & 2.28 & 88478.5 & 0.085 \\
\hline 2.31 & 61666.8 & 0.698 & 2.37 & 77277.7 & 0.301 & 2.31 & 88236.8 & 0.049 \\
\hline 2.34 & 61060.5 & 0.691 & 2.40 & 77044.7 & 0.285 & 2.34 & 88020.6 & 0.016 \\
\hline 2.37 & 60484.0 & 0.682 & 2.43 & 76832.6 & 0.271 & 2.37 & 87827.1 & -0.013 \\
\hline 2.40 & 59935.9 & 0.671 & 2.46 & 76639.6 & 0.259 & 2.40 & 87653.9 & -0.039 \\
\hline 2.43 & 59415.1 & 0.660 & 2.49 & 76464.0 & 0.249 & 2.43 & 87498.9 & -0.062 \\
\hline 2.46 & 58920.7 & 0.647 & 2.52 & 76304.4 & 0.240 & 2.46 & 87359.5 & -0.084 \\
\hline 2.49 & 58451.2 & 0.633 & 2.55 & 76159.5 & 0.233 & 2.49 & 87234.9 & -0.101 \\
\hline 2.52 & 58005.7 & 0.619 & 2.58 & 76027.9 & 0.227 & 2.52 & 87123.0 & -0.115 \\
\hline 2.55 & 57583.1 & 0.605 & 2.61 & 75908.5 & 0.223 & 2.55 & 87022.4 & -0.127 \\
\hline 2.58 & 57182.5 & 0.590 & 2.64 & 75800.5 & 0.219 & 2.58 & 86931.7 & -0.136 \\
\hline 2.61 & 56802.8 & 0.574 & 2.67 & 75702.7 & 0.217 & 2.61 & 86849.8 & -0.143 \\
\hline 2.64 & 56443.1 & 0.559 & 2.70 & 75614.3 & 0.215 & 2.64 & 86775.7 & -0.148 \\
\hline 2.67 & 56102.3 & 0.543 & 2.73 & 75534.6 & 0.214 & 2.67 & 86708.2 & -0.151 \\
\hline 2.70 & 55779.7 & 0.527 & 2.76 & 75462.8 & 0.214 & 2.70 & 86646.6 & -0.153 \\
\hline 2.73 & 55474.3 & 0.512 & 2.79 & 75398.2 & 0.214 & 2.73 & 86590.0 & -0.153 \\
\hline 2.76 & 55185.3 & 0.496 & 2.82 & 75340.3 & 0.215 & 2.76 & 86537.6 & -0.152 \\
\hline 2.79 & 54911.9 & 0.480 & 2.85 & 75288.4 & 0.216 & 2.79 & 86488.8 & -0.150 \\
\hline 2.82 & 54653.3 & 0.465 & 2.88 & 75230.5 & 0.221 & 2.82 & 86443.0 & -0.147 \\
\hline 2.85 & 54408.7 & 0.450 & 2.91 & 75189.8 & 0.223 & 2.85 & 86399.7 & -0.143 \\
\hline 2.88 & 54189.1 & 0.437 & 2.94 & 75153.7 & 0.226 & 2.88 & 86358.8 & -0.141 \\
\hline 2.91 & 53970.1 & 0.422 & 2.97 & 75121.8 & 0.228 & 2.91 & 86319.5 & -0.138 \\
\hline 2.94 & 53763.2 & 0.407 & 3.00 & 75093.9 & 0.231 & 2.94 & 86281.6 & -0.134 \\
\hline
\end{tabular}




\begin{tabular}{|c|c|c|c|c|c|c|c|c|}
\hline 2.97 & 53567.7 & 0.393 & 3.03 & 75069.5 & 0.235 & 2.97 & 86244.4 & -0.130 \\
\hline 3.00 & 53383.0 & 0.379 & 3.06 & 75048.3 & 0.238 & 3.00 & 86208.1 & -0.124 \\
\hline 3.03 & 53208.6 & 0.365 & 3.09 & 75030.1 & 0.242 & 3.03 & 86172.2 & -0.119 \\
\hline 3.06 & 53043.9 & 0.352 & 3.12 & 75014.7 & 0.246 & 3.06 & 86136.5 & -0.114 \\
\hline 3.09 & 52888.4 & 0.339 & 3.15 & 75001.7 & 0.250 & 3.09 & 86101.0 & -0.108 \\
\hline 3.12 & 52741.7 & 0.326 & 3.18 & 74991.0 & 0.254 & 3.12 & 86065.4 & -0.101 \\
\hline 3.15 & 52603.2 & 0.313 & 3.21 & 74982.3 & 0.258 & 3.15 & 86027.3 & -0.095 \\
\hline 3.18 & 52472.6 & 0.301 & 3.27 & 74970.4 & 0.266 & 3.21 & 85950.8 & -0.088 \\
\hline 3.21 & 52349.4 & 0.289 & 3.30 & 74966.8 & 0.270 & 3.24 & 85914.2 & -0.086 \\
\hline 3.24 & 52233.1 & 0.278 & 3.33 & 74964.6 & 0.274 & 3.27 & 85877.4 & -0.084 \\
\hline 3.27 & 52123.5 & 0.267 & 3.36 & 74963.6 & 0.278 & 3.30 & 85840.4 & -0.082 \\
\hline 3.30 & 52020.2 & 0.256 & 3.39 & 74963.6 & 0.282 & 3.33 & 85803.0 & -0.081 \\
\hline 3.33 & 51922.9 & 0.245 & 3.42 & 74964.7 & 0.286 & 3.36 & 85765.6 & -0.080 \\
\hline 3.36 & 51831.1 & 0.235 & 3.45 & 74966.7 & 0.290 & 3.39 & 85728.0 & -0.080 \\
\hline 3.39 & 51744.7 & 0.225 & 3.48 & 74969.4 & 0.294 & 3.42 & 85690.4 & -0.080 \\
\hline 3.42 & 51663.2 & 0.215 & 3.51 & 74972.9 & 0.297 & 3.45 & 85652.9 & -0.081 \\
\hline 3.45 & 51586.5 & 0.206 & 3.54 & 74976.9 & 0.301 & 3.48 & 85615.1 & -0.083 \\
\hline 3.48 & 51514.3 & 0.197 & 3.57 & 74981.5 & 0.304 & 3.51 & 85577.3 & -0.084 \\
\hline 3.51 & 51446.2 & 0.188 & 3.60 & 74986.5 & 0.307 & 3.54 & 85539.6 & -0.086 \\
\hline 3.54 & 51382.2 & 0.180 & 3.63 & 74991.9 & 0.310 & 3.57 & 85502.1 & -0.088 \\
\hline 3.57 & 51322.0 & 0.172 & 3.66 & 74997.7 & 0.313 & 3.60 & 85464.7 & -0.090 \\
\hline 3.60 & 51265.3 & 0.164 & 3.69 & 75003.7 & 0.316 & 3.63 & 85427.5 & -0.092 \\
\hline 3.63 & 51211.9 & 0.157 & 3.72 & 75009.9 & 0.319 & 3.69 & 85199.5 & -0.097 \\
\hline 3.66 & 51161.8 & 0.149 & 3.75 & 75016.4 & 0.321 & 3.72 & 85184.4 & -0.100 \\
\hline 3.69 & 51114.6 & 0.142 & 3.78 & 75023.0 & 0.323 & 3.75 & 85189.9 & -0.103 \\
\hline 3.72 & 51070.4 & 0.135 & 3.81 & 75029.6 & 0.325 & 3.78 & 85162.0 & -0.106 \\
\hline 3.75 & 51028.8 & 0.129 & 3.84 & 75036.2 & 0.327 & 3.81 & 85135.8 & -0.109 \\
\hline 3.78 & 50989.6 & 0.123 & 3.87 & 75042.9 & 0.329 & 3.84 & 85106.9 & -0.112 \\
\hline 3.81 & 50952.9 & 0.117 & 3.90 & 75049.6 & 0.330 & 3.87 & 85076.5 & -0.114 \\
\hline 3.84 & 50918.3 & 0.111 & 3.93 & 75056.2 & 0.331 & 3.90 & 85048.8 & -0.117 \\
\hline 3.87 & 50885.9 & 0.105 & 3.96 & 75062.7 & 0.332 & 3.93 & 85020.2 & -0.119 \\
\hline 3.90 & 50855.5 & 0.100 & 3.99 & 75069.2 & 0.333 & 3.96 & 84991.1 & -0.121 \\
\hline 3.93 & 50827.0 & 0.095 & 4.02 & 75075.5 & 0.334 & 3.99 & 84963.8 & -0.122 \\
\hline 3.96 & 50801.0 & 0.090 & 4.05 & 75081.6 & 0.334 & 4.02 & 84935.3 & -0.124 \\
\hline 3.99 & 50775.9 & 0.086 & 4.08 & 75087.6 & 0.334 & 4.05 & 84909.4 & -0.125 \\
\hline 4.00 & 50768.0 & 0.084 & 4.11 & 75093.5 & 0.334 & 4.08 & 84881.7 & -0.126 \\
\hline 4.03 & 50745.0 & 0.080 & 4.14 & 75099.1 & 0.334 & 4.11 & 84855.8 & -0.127 \\
\hline 4.06 & 50723.5 & 0.076 & 4.17 & 75104.5 & 0.334 & 4.14 & 84830.4 & -0.127 \\
\hline 4.09 & 50703.4 & 0.072 & 4.20 & 75109.6 & 0.333 & 4.17 & 84805.1 & -0.127 \\
\hline 4.12 & 50684.6 & 0.068 & 4.23 & 75114.5 & 0.333 & 4.20 & 84780.9 & -0.127 \\
\hline 4.15 & 50667.1 & 0.064 & 4.26 & 75119.2 & 0.332 & 4.23 & 84757.6 & -0.127 \\
\hline 4.18 & 50650.7 & 0.061 & 4.29 & 75123.6 & 0.331 & 4.26 & 84734.8 & -0.127 \\
\hline 4.21 & 50635.4 & 0.058 & 4.32 & 75127.7 & 0.329 & 4.29 & 84712.7 & -0.126 \\
\hline 4.24 & 50621.1 & 0.054 & 4.35 & 75131.5 & 0.328 & 4.32 & 84691.2 & -0.125 \\
\hline
\end{tabular}




\begin{tabular}{|c|c|c|c|c|c|c|c|c|}
\hline 4.27 & 50607.9 & 0.051 & 4.38 & 75135.0 & 0.326 & 4.35 & 84670.5 & -0.124 \\
\hline 4.30 & 50595.5 & 0.049 & 4.41 & 75138.2 & 0.324 & 4.38 & 84650.4 & -0.123 \\
\hline 4.33 & 50584.0 & 0.046 & 4.44 & 75141.0 & 0.323 & 4.41 & 84631.5 & -0.121 \\
\hline 4.36 & 50573.3 & 0.043 & 4.48 & 75143.6 & 0.321 & 4.44 & 84613.0 & -0.120 \\
\hline 4.39 & 50563.3 & 0.041 & & & & 4.48 & 84595.0 & -0.118 \\
\hline 4.42 & 50554.1 & 0.038 & & & & & & \\
\hline 4.45 & 50545.6 & 0.036 & & & & & & \\
\hline 4.48 & 50537.6 & 0.034 & & & & & & \\
\hline
\end{tabular}

\begin{tabular}{|c|c|c|c|c|c|c|c|c|}
\hline \multicolumn{3}{|c|}{$(5)^{4} \Sigma^{+}$} & \multicolumn{3}{|c|}{$(1)^{4} \Pi$} & \multicolumn{3}{|c|}{$(2)^{4} \Pi$} \\
\hline $\mathrm{R}(\AA)$ & $\mathrm{E}\left(\mathrm{cm}^{-1}\right)$ & $\mu$ (a.u.) & $\mathrm{R}(\AA)$ & $\mathrm{E}\left(\mathrm{cm}^{-1}\right)$ & $\mu$ (a.u.) & $\mathrm{R}(\AA)$ & $\mathrm{E}\left(\mathrm{cm}^{-1}\right)$ & $\mu$ (a.u.) \\
\hline 1.41 & 152401.4 & -0.920 & 1.41 & 108606.2 & 1.080 & 1.41 & 119107.6 & -0.455 \\
\hline 1.44 & 145376.8 & -0.851 & 1.44 & 102527.0 & 1.078 & 1.44 & 112002.3 & -0.401 \\
\hline 1.47 & 139284.3 & -0.786 & 1.47 & 97168.0 & 1.070 & 1.47 & 105802.4 & -0.350 \\
\hline 1.50 & 133998.1 & -0.721 & 1.50 & 92437.0 & 1.059 & 1.50 & 100386.4 & -0.300 \\
\hline 1.53 & 129408.7 & -0.658 & 1.53 & 88253.8 & 1.043 & 1.53 & 95649.0 & -0.251 \\
\hline 1.56 & 125420.0 & -0.597 & 1.56 & 84548.7 & 1.025 & 1.56 & 91498.5 & -0.202 \\
\hline 1.59 & 121954.4 & -0.535 & 1.59 & 81260.9 & 1.005 & 1.59 & 87855.5 & -0.153 \\
\hline 1.62 & 118943.1 & -0.473 & 1.62 & 78337.6 & 0.982 & 1.62 & 84651.4 & -0.104 \\
\hline 1.65 & 116326.9 & -0.411 & 1.65 & 75732.9 & 0.958 & 1.65 & 81826.9 & -0.054 \\
\hline 1.68 & 114050.8 & -0.350 & 1.68 & 73407.2 & 0.932 & 1.68 & 79330.8 & -0.004 \\
\hline 1.71 & 112066.0 & -0.288 & 1.71 & 71326.1 & 0.906 & 1.71 & 77118.9 & 0.046 \\
\hline 1.74 & 110331.5 & -0.228 & 1.74 & 69460.0 & 0.878 & 1.74 & 75153.1 & 0.096 \\
\hline 1.77 & 108814.2 & -0.169 & 1.77 & 67783.1 & 0.850 & 1.77 & 73400.8 & 0.146 \\
\hline 1.80 & 107485.2 & -0.111 & 1.80 & 66273.2 & 0.822 & 1.80 & 71833.2 & 0.195 \\
\hline 1.83 & 106319.2 & -0.055 & 1.83 & 64910.9 & 0.794 & 1.83 & 70425.9 & 0.243 \\
\hline 1.86 & 105295.4 & -0.001 & 1.86 & 63679.7 & 0.765 & 1.86 & 69158.1 & 0.290 \\
\hline 1.89 & 104395.1 & 0.050 & 1.89 & 62564.8 & 0.737 & 1.89 & 68011.3 & 0.334 \\
\hline 1.92 & 103602.3 & 0.100 & 1.92 & 61553.6 & 0.708 & 1.92 & 66969.9 & 0.377 \\
\hline 1.95 & 102901.7 & 0.146 & 1.95 & 60635.0 & 0.680 & 1.95 & 66020.1 & 0.417 \\
\hline 1.98 & 102282.2 & 0.190 & 1.98 & 59799.2 & 0.653 & 1.98 & 65150.3 & 0.454 \\
\hline 2.01 & 101732.8 & 0.231 & 2.01 & 59037.7 & 0.625 & 2.01 & 64350.3 & 0.488 \\
\hline 2.04 & 101244.3 & 0.270 & 2.04 & 58342.8 & 0.598 & 2.04 & 63611.4 & 0.518 \\
\hline 2.07 & 100808.8 & 0.304 & 2.07 & 57707.9 & 0.572 & 2.07 & 62926.1 & 0.546 \\
\hline 2.10 & 100418.7 & 0.336 & 2.10 & 57127.1 & 0.546 & 2.10 & 62288.0 & 0.570 \\
\hline 2.13 & 100068.3 & 0.365 & 2.13 & 56595.2 & 0.521 & 2.13 & 61691.8 & 0.591 \\
\hline 2.16 & 99751.7 & 0.391 & 2.16 & 56107.5 & 0.497 & 2.16 & 61132.6 & 0.608 \\
\hline 2.19 & 99464.5 & 0.413 & 2.19 & 55659.9 & 0.473 & 2.19 & 60606.6 & 0.622 \\
\hline 2.22 & 99202.3 & 0.433 & 2.22 & 55248.8 & 0.450 & 2.22 & 60110.4 & 0.633 \\
\hline 2.25 & 98961.9 & 0.449 & 2.25 & 54870.7 & 0.428 & 2.25 & 59641.2 & 0.641 \\
\hline 2.28 & 98740.5 & 0.463 & 2.28 & 54522.9 & 0.407 & 2.28 & 59196.5 & 0.646 \\
\hline 2.31 & 98535.4 & 0.475 & 2.31 & 54202.6 & 0.386 & 2.31 & 58774.4 & 0.649 \\
\hline 2.34 & 98344.8 & 0.484 & 2.34 & 53907.6 & 0.366 & 2.34 & 58373.0 & 0.650 \\
\hline 2.37 & 98166.9 & 0.490 & 2.37 & 53635.6 & 0.347 & 2.37 & 57990.9 & 0.649 \\
\hline
\end{tabular}




\begin{tabular}{|c|c|c|c|c|c|c|c|c|}
\hline 2.40 & 98000.1 & 0.495 & 2.40 & 53384.9 & 0.328 & 2.40 & 57626.7 & 0.646 \\
\hline 2.43 & 97843.3 & 0.498 & 2.43 & 53153.6 & 0.311 & 2.43 & 57279.4 & 0.641 \\
\hline 2.46 & 97695.3 & 0.499 & 2.46 & 52940.2 & 0.294 & 2.46 & 56947.9 & 0.635 \\
\hline 2.49 & 97555.2 & 0.499 & 2.49 & 52743.3 & 0.278 & 2.49 & 56631.4 & 0.627 \\
\hline 2.52 & 97422.3 & 0.497 & 2.52 & 52561.6 & 0.262 & 2.52 & 56329.0 & 0.618 \\
\hline 2.55 & 97295.9 & 0.494 & 2.55 & 52393.8 & 0.248 & 2.55 & 56040.2 & 0.609 \\
\hline 2.58 & 97175.4 & 0.490 & 2.58 & 52239.0 & 0.234 & 2.58 & 55764.2 & 0.598 \\
\hline 2.61 & 97060.5 & 0.485 & 2.61 & 52096.2 & 0.220 & 2.61 & 55500.5 & 0.587 \\
\hline 2.64 & 96950.5 & 0.479 & 2.64 & 51964.4 & 0.208 & 2.64 & 55248.4 & 0.576 \\
\hline 2.67 & 96845.3 & 0.472 & 2.67 & 51842.7 & 0.196 & 2.67 & 55007.6 & 0.563 \\
\hline 2.70 & 96744.4 & 0.464 & 2.70 & 51730.5 & 0.184 & 2.70 & 54777.5 & 0.551 \\
\hline 2.73 & 96647.7 & 0.456 & 2.73 & 51627.0 & 0.173 & 2.73 & 54557.7 & 0.538 \\
\hline 2.76 & 96554.9 & 0.448 & 2.76 & 51531.6 & 0.163 & 2.76 & 54347.8 & 0.525 \\
\hline 2.79 & 96465.7 & 0.439 & 2.79 & 51443.6 & 0.153 & 2.79 & 54147.2 & 0.512 \\
\hline 2.82 & 96380.1 & 0.430 & 2.82 & 51362.7 & 0.144 & 2.82 & 53955.8 & 0.498 \\
\hline 2.85 & 96297.9 & 0.420 & 2.85 & 51288.0 & 0.136 & 2.85 & 53773.2 & 0.484 \\
\hline 2.88 & 96214.9 & 0.409 & 2.88 & 51227.0 & 0.131 & 2.88 & 53597.7 & 0.470 \\
\hline 2.91 & 96139.0 & 0.399 & 2.91 & 51163.8 & 0.123 & 2.91 & 53431.4 & 0.457 \\
\hline 2.94 & 96066.2 & 0.389 & 2.94 & 51105.8 & 0.116 & 2.94 & 53272.6 & 0.443 \\
\hline 2.97 & 95996.0 & 0.379 & 2.97 & 51052.5 & 0.109 & 2.97 & 53121.2 & 0.430 \\
\hline 3.00 & 95928.8 & 0.369 & 3.00 & 51003.8 & 0.102 & 3.00 & 52976.9 & 0.417 \\
\hline 3.03 & 95864.4 & 0.359 & 3.03 & 50959.1 & 0.096 & 3.03 & 52839.3 & 0.404 \\
\hline 3.06 & 95802.6 & 0.349 & 3.06 & 50918.2 & 0.090 & 3.06 & 52708.2 & 0.391 \\
\hline 3.09 & 95743.3 & 0.339 & 3.09 & 50880.7 & 0.085 & 3.09 & 52583.5 & 0.378 \\
\hline 3.12 & 95686.5 & 0.329 & 3.12 & 50846.5 & 0.080 & 3.12 & 52464.8 & 0.365 \\
\hline 3.15 & 95631.5 & 0.319 & 3.15 & 50815.2 & 0.075 & 3.15 & 52352.1 & 0.353 \\
\hline 3.18 & 95581.0 & 0.309 & 3.18 & 50786.9 & 0.071 & 3.18 & 52245.2 & 0.341 \\
\hline 3.21 & 95531.6 & 0.299 & 3.21 & 50760.7 & 0.066 & 3.21 & 52143.4 & 0.329 \\
\hline 3.24 & 95484.4 & 0.290 & 3.24 & 50736.7 & 0.062 & 3.24 & 52046.8 & 0.317 \\
\hline 3.27 & 95439.3 & 0.280 & 3.27 & 50714.7 & 0.059 & 3.27 & 51955.1 & 0.306 \\
\hline 3.30 & 95396.2 & 0.271 & 3.30 & 50694.6 & 0.055 & 3.30 & 51868.1 & 0.295 \\
\hline 3.33 & 95355.2 & 0.262 & 3.33 & 50676.2 & 0.052 & 3.33 & 51785.7 & 0.284 \\
\hline 3.36 & 95315.9 & 0.254 & 3.36 & 50659.5 & 0.049 & 3.36 & 51707.6 & 0.273 \\
\hline 3.39 & 95278.4 & 0.245 & 3.39 & 50644.1 & 0.046 & 3.39 & 51633.6 & 0.263 \\
\hline 3.42 & 95242.7 & 0.237 & 3.42 & 50630.1 & 0.044 & 3.42 & 51563.6 & 0.253 \\
\hline 3.45 & 95208.7 & 0.229 & 3.45 & 50617.4 & 0.042 & 3.45 & 51497.2 & 0.243 \\
\hline 3.48 & 95176.4 & 0.221 & 3.48 & 50605.7 & 0.039 & 3.48 & 51434.5 & 0.234 \\
\hline 3.51 & 95145.6 & 0.213 & 3.51 & 50595.1 & 0.037 & 3.51 & 51375.1 & 0.224 \\
\hline 3.54 & 95116.4 & 0.205 & 3.54 & 50585.3 & 0.036 & 3.54 & 51319.0 & 0.215 \\
\hline 3.57 & 95088.5 & 0.198 & 3.57 & 50576.4 & 0.034 & 3.57 & 51265.9 & 0.206 \\
\hline 3.60 & 95062.1 & 0.191 & 3.60 & 50568.3 & 0.032 & 3.60 & 51215.8 & 0.198 \\
\hline 3.63 & 95037.0 & 0.184 & 3.63 & 50560.9 & 0.031 & 3.63 & 51168.4 & 0.189 \\
\hline 3.66 & 95012.7 & 0.177 & 3.66 & 50554.1 & 0.030 & 3.66 & 51123.7 & 0.181 \\
\hline 3.69 & 94990.0 & 0.170 & 3.69 & 50547.9 & 0.029 & 3.69 & 51081.6 & 0.173 \\
\hline
\end{tabular}




\begin{tabular}{|c|c|c|c|c|c|c|c|c|}
\hline 3.72 & 94968.5 & 0.164 & 3.72 & 50542.2 & 0.028 & 3.72 & 51041.8 & 0.166 \\
\hline 3.75 & 94949.2 & 0.158 & 3.75 & 50537.0 & 0.027 & 3.75 & 51004.3 & 0.158 \\
\hline 3.78 & 94929.9 & 0.152 & 3.78 & 50533.4 & 0.026 & 3.78 & 50969.2 & 0.151 \\
\hline 3.81 & 94911.6 & 0.146 & 3.81 & 50529.0 & 0.025 & 3.81 & 50936.0 & 0.144 \\
\hline 3.84 & 94894.3 & 0.140 & 3.84 & 50524.9 & 0.025 & 3.84 & 50904.7 & 0.137 \\
\hline 3.87 & 94878.0 & 0.135 & 3.87 & 50521.3 & 0.024 & 3.87 & 50875.3 & 0.130 \\
\hline 3.90 & 94862.6 & 0.130 & 3.90 & 50517.9 & 0.024 & 3.90 & 50847.7 & 0.124 \\
\hline 3.93 & 94848.1 & 0.124 & 3.93 & 50514.7 & 0.024 & 3.93 & 50821.8 & 0.117 \\
\hline 3.96 & 94834.4 & 0.119 & 3.96 & 50512.4 & 0.024 & 3.96 & 50798.1 & 0.111 \\
\hline 3.99 & 94821.6 & 0.115 & 3.99 & 50509.7 & 0.024 & 3.99 & 50775.3 & 0.105 \\
\hline 4.02 & 94809.6 & 0.110 & 4.00 & 50508.4 & 0.024 & 4.00 & 50767.3 & 0.103 \\
\hline 4.05 & 94798.3 & 0.105 & 4.03 & 50506.4 & 0.024 & 4.03 & 50747.2 & 0.097 \\
\hline 4.08 & 94787.8 & 0.101 & 4.06 & 50504.1 & 0.024 & 4.06 & 50727.8 & 0.092 \\
\hline 4.11 & 94777.9 & 0.097 & 4.09 & 50501.9 & 0.024 & 4.09 & 50709.6 & 0.086 \\
\hline 4.14 & 94768.7 & 0.093 & 4.12 & 50499.9 & 0.025 & 4.12 & 50692.7 & 0.081 \\
\hline 4.17 & 94760.1 & 0.089 & 4.15 & 50498.0 & 0.025 & 4.15 & 50677.0 & 0.075 \\
\hline 4.20 & 94752.2 & 0.086 & 4.18 & 50496.2 & 0.026 & 4.18 & 50662.4 & 0.070 \\
\hline 4.23 & 94744.8 & 0.082 & 4.21 & 50494.5 & 0.026 & 4.21 & 50648.8 & 0.065 \\
\hline 4.26 & 94738.0 & 0.079 & 4.24 & 50492.8 & 0.027 & 4.24 & 50636.2 & 0.060 \\
\hline 4.29 & 94731.8 & 0.075 & 4.27 & 50491.2 & 0.028 & 4.27 & 50624.6 & 0.055 \\
\hline 4.32 & 94726.0 & 0.072 & 4.30 & 50489.8 & 0.028 & 4.30 & 50613.9 & 0.051 \\
\hline 4.35 & 94720.6 & 0.069 & 4.33 & 50488.3 & 0.029 & 4.33 & 50604.0 & 0.046 \\
\hline 4.38 & 94715.6 & 0.066 & 4.36 & 50486.9 & 0.029 & 4.36 & 50595.0 & 0.042 \\
\hline 4.41 & 94711.0 & 0.064 & 4.39 & 50485.5 & 0.030 & 4.39 & 50586.6 & 0.038 \\
\hline 4.44 & 94706.8 & 0.061 & 4.42 & 50484.1 & 0.030 & 4.42 & 50579.0 & 0.035 \\
\hline \multirow[t]{2}{*}{4.48} & 94702.9 & 0.058 & 4.45 & 50482.8 & 0.031 & 4.45 & 50572.0 & 0.031 \\
\hline & & & 4.48 & 50481.5 & 0.031 & 4.48 & 50565.7 & 0.028 \\
\hline
\end{tabular}

\begin{tabular}{|c|c|c|c|c|c|c|c|c|}
\hline \multicolumn{3}{|c|}{$(3)^{4} \Pi$} & \multicolumn{3}{|c|}{$(4)^{4} \Pi$} & \multicolumn{3}{|c|}{$(5)^{4} \Pi$} \\
\hline $\mathrm{R}(\AA)$ & $\mathrm{E}\left(\mathrm{cm}^{-1}\right)$ & $\mu$ (a.u.) & $\mathrm{R}(\AA)$ & $\mathrm{E}\left(\mathrm{cm}^{-1}\right)$ & $\mu$ (a.u.) & $\mathrm{R}(\AA)$ & $\mathrm{E}\left(\mathrm{cm}^{-1}\right)$ & $\mu$ (a.u.) \\
\hline 1.41 & 129873.5 & 1.683 & 1.41 & 142350.1 & 0.414 & 1.41 & 144373.2 & 2.352 \\
\hline 1.44 & 122817.9 & 1.660 & 1.44 & 135352.2 & 0.424 & 1.44 & 137028.9 & 2.270 \\
\hline 1.47 & 116664.1 & 1.637 & 1.47 & 129275.0 & 0.431 & 1.47 & 130518.6 & 2.187 \\
\hline 1.50 & 111294.0 & 1.613 & 1.50 & 123997.9 & 0.437 & 1.50 & 124753.9 & 2.104 \\
\hline 1.53 & 106604.7 & 1.588 & 1.53 & 119415.0 & 0.448 & 1.53 & 119649.8 & 2.017 \\
\hline 1.56 & 102506.5 & 1.561 & 1.56 & 115122.4 & 1.931 & 1.56 & 115441.0 & 0.459 \\
\hline 1.59 & 98921.5 & 1.533 & 1.59 & 111112.9 & 1.862 & 1.59 & 111988.1 & 0.445 \\
\hline 1.62 & 95782.3 & 1.504 & 1.62 & 107554.6 & 1.749 & 1.62 & 108992.9 & 0.444 \\
\hline 1.65 & 93030.4 & 1.473 & 1.65 & 104400.0 & 1.637 & 1.65 & 106396.0 & 0.443 \\
\hline 1.68 & 90615.3 & 1.440 & 1.68 & 102200.0 & 1.543 & 1.68 & 104146.0 & 0.442 \\
\hline 1.71 & 88493.3 & 1.406 & 1.71 & 100200.0 & 1.464 & 1.71 & 102199.1 & 0.440 \\
\hline 1.74 & 86626.7 & 1.370 & 1.73 & 98700.0 & 1.403 & 1.74 & 100517.1 & 0.438 \\
\hline 1.77 & 84982.8 & 1.332 & 1.77 & 96519.2 & 1.312 & 1.77 & 99066.9 & 0.435 \\
\hline 1.80 & 83533.5 & 1.292 & 1.80 & 94873.2 & 1.237 & 1.80 & 97819.6 & 0.431 \\
\hline
\end{tabular}




\begin{tabular}{|c|c|c|c|c|c|c|c|c|}
\hline 1.83 & 82254.4 & 1.250 & 1.83 & 93453.6 & 1.159 & 1.83 & 96750.1 & 0.427 \\
\hline 1.86 & 81124.4 & 1.207 & 1.86 & 92098.0 & 1.096 & 1.86 & 95836.7 & 0.423 \\
\hline 1.89 & 80125.4 & 1.162 & 1.89 & 90901.9 & 1.035 & 1.89 & 95060.1 & 0.418 \\
\hline 1.92 & 79241.7 & 1.115 & 1.92 & 89885.8 & 0.976 & 1.92 & 94403.5 & 0.413 \\
\hline 1.95 & 78459.6 & 1.068 & 1.95 & 89016.3 & 0.926 & 1.95 & 93851.8 & 0.408 \\
\hline 1.98 & 77767.5 & 1.020 & 1.98 & 88179.1 & 0.878 & 1.98 & 93391.9 & 0.402 \\
\hline 2.01 & 77155.0 & 0.972 & 2.01 & 87499.8 & 0.829 & 2.01 & 93012.1 & 0.395 \\
\hline 2.04 & 76613.4 & 0.924 & 2.04 & 86913.2 & 0.778 & 2.04 & 92702.0 & 0.389 \\
\hline 2.07 & 76134.8 & 0.877 & 2.07 & 86409.2 & 0.730 & 2.07 & 92452.5 & 0.381 \\
\hline 2.10 & 75712.6 & 0.831 & 2.10 & 85979.7 & 0.688 & 2.10 & 92255.5 & 0.374 \\
\hline 2.13 & 75340.6 & 0.787 & 2.13 & 85648.4 & 0.635 & 2.13 & 92103.8 & 0.366 \\
\hline 2.16 & 75013.6 & 0.745 & 2.16 & 85291.7 & 0.596 & 2.16 & 91991.0 & 0.358 \\
\hline 2.19 & 74726.9 & 0.704 & 2.19 & 85053.1 & 0.557 & 2.19 & 91911.5 & 0.350 \\
\hline 2.22 & 74476.2 & 0.666 & 2.22 & 84824.2 & 0.530 & 2.22 & 91860.3 & 0.342 \\
\hline 2.25 & 74257.7 & 0.630 & 2.25 & 84647.7 & 0.504 & 2.25 & 91833.1 & 0.333 \\
\hline 2.28 & 74067.9 & 0.597 & 2.28 & 84495.1 & 0.483 & 2.28 & 91826.2 & 0.324 \\
\hline 2.31 & 73904.0 & 0.566 & 2.31 & 84388.2 & 0.452 & 2.31 & 91836.2 & 0.316 \\
\hline 2.34 & 73763.1 & 0.537 & 2.34 & 84295.6 & 0.426 & 2.34 & 91860.3 & 0.307 \\
\hline 2.37 & 73642.7 & 0.511 & 2.37 & 84237.4 & 0.396 & 2.37 & 91895.9 & 0.298 \\
\hline 2.40 & 73540.6 & 0.486 & 2.40 & 84185.0 & 0.381 & 2.40 & 91941.0 & 0.289 \\
\hline 2.43 & 73454.8 & 0.464 & 2.43 & 84151.1 & 0.362 & 2.43 & 91993.7 & 0.280 \\
\hline 2.46 & 73383.5 & 0.444 & 2.46 & 84131.3 & 0.343 & 2.46 & 92052.3 & 0.271 \\
\hline 2.49 & 73325.2 & 0.425 & 2.49 & 84123.1 & 0.325 & 2.49 & 92115.5 & 0.262 \\
\hline 2.52 & 73278.3 & 0.408 & 2.52 & 84124.7 & 0.308 & 2.52 & 92182.1 & 0.253 \\
\hline 2.55 & 73241.6 & 0.393 & 2.55 & 84134.4 & 0.292 & 2.55 & 92251.0 & 0.244 \\
\hline 2.58 & 73214.0 & 0.379 & 2.58 & 84150.9 & 0.278 & 2.58 & 92321.5 & 0.235 \\
\hline 2.61 & 73194.3 & 0.367 & 2.61 & 84172.9 & 0.264 & 2.61 & 92392.8 & 0.227 \\
\hline 2.64 & 73181.7 & 0.356 & 2.64 & 84199.3 & 0.250 & 2.64 & 92464.4 & 0.219 \\
\hline 2.67 & 73175.3 & 0.346 & 2.67 & 84231.5 & 0.235 & 2.67 & 92535.6 & 0.210 \\
\hline 2.70 & 73174.5 & 0.337 & 2.69 & 84250.6 & 0.230 & 2.70 & 92606.7 & 0.202 \\
\hline 2.73 & 73178.4 & 0.329 & 2.72 & 84283.5 & 0.216 & 2.73 & 92676.4 & 0.195 \\
\hline 2.76 & 73186.6 & 0.322 & 2.76 & 84331.2 & 0.199 & 2.76 & 92744.9 & 0.187 \\
\hline 2.79 & 73198.6 & 0.316 & 2.79 & 84366.9 & 0.188 & 2.79 & 92811.9 & 0.180 \\
\hline 2.82 & 73213.9 & 0.311 & 2.82 & 84402.7 & 0.177 & 2.82 & 92877.4 & 0.173 \\
\hline 2.85 & 73231.9 & 0.306 & 2.85 & 84438.0 & 0.166 & 2.85 & 92941.0 & 0.166 \\
\hline 2.88 & 73254.3 & 0.302 & 2.88 & 84477.9 & 0.156 & 2.88 & 93005.8 & 0.159 \\
\hline 2.91 & 73276.9 & 0.298 & 2.91 & 84511.1 & 0.147 & 2.91 & 93065.5 & 0.152 \\
\hline 2.94 & 73301.5 & 0.295 & 2.94 & 84542.9 & 0.138 & 2.94 & 93123.4 & 0.146 \\
\hline 2.97 & 73327.6 & 0.293 & 2.97 & 84573.1 & 0.130 & 2.97 & 93179.3 & 0.140 \\
\hline 3.00 & 73355.2 & 0.291 & 3.00 & 84601.8 & 0.121 & 3.00 & 93233.4 & 0.134 \\
\hline 3.03 & 73383.9 & 0.290 & 3.03 & 84627.0 & 0.115 & 3.03 & 93285.6 & 0.128 \\
\hline 3.06 & 73413.6 & 0.289 & 3.06 & 84653.2 & 0.107 & 3.06 & 93336.2 & 0.123 \\
\hline 3.09 & 73444.1 & 0.288 & 3.09 & 84672.9 & 0.099 & 3.09 & 93384.7 & 0.118 \\
\hline 3.12 & 73475.4 & 0.288 & 3.12 & 84699.8 & 0.091 & 3.12 & 93431.5 & 0.113 \\
\hline
\end{tabular}




\begin{tabular}{|c|c|c|c|c|c|c|c|c|}
\hline 3.15 & 73507.1 & 0.288 & 3.15 & 84713.5 & 0.086 & 3.15 & 93476.7 & 0.108 \\
\hline 3.18 & 73539.6 & 0.289 & 3.18 & 84727.5 & 0.078 & 3.18 & 93520.1 & 0.104 \\
\hline 3.21 & 73572.0 & 0.289 & 3.21 & 84742.6 & 0.072 & 3.21 & 93561.9 & 0.099 \\
\hline 3.24 & 73604.7 & 0.290 & 3.24 & 84754.0 & 0.064 & 3.24 & 93602.2 & 0.095 \\
\hline 3.27 & 73637.4 & 0.291 & 3.27 & 84763.5 & 0.057 & 3.27 & 93641.1 & 0.091 \\
\hline 3.30 & 73670.2 & 0.292 & 3.30 & 84771.0 & 0.049 & 3.30 & 93678.4 & 0.088 \\
\hline 3.33 & 73702.9 & 0.293 & 3.33 & 84777.1 & 0.042 & 3.33 & 93714.2 & 0.084 \\
\hline 3.36 & 73735.4 & 0.295 & 3.36 & 84780.0 & 0.035 & 3.36 & 93748.8 & 0.080 \\
\hline 3.39 & 73767.8 & 0.296 & 3.39 & 84782.0 & 0.030 & 3.39 & 93782.0 & 0.077 \\
\hline 3.42 & 73799.9 & 0.298 & 3.42 & 84783.1 & 0.028 & 3.42 & 93813.8 & 0.074 \\
\hline 3.45 & 73831.7 & 0.300 & 3.45 & 84785.9 & 0.027 & 3.45 & 93844.5 & 0.071 \\
\hline 3.48 & 73863.2 & 0.301 & 3.48 & 84809.9 & 0.026 & 3.48 & 93873.9 & 0.068 \\
\hline 3.51 & 73895.1 & 0.304 & 3.51 & 84783.6 & 0.027 & 3.51 & 93902.2 & 0.065 \\
\hline 3.54 & 73925.8 & 0.305 & 3.54 & 84778.4 & 0.029 & 3.54 & 93929.4 & 0.063 \\
\hline 3.57 & 73956.0 & 0.307 & 3.57 & 84771.8 & 0.031 & 3.57 & 93955.6 & 0.060 \\
\hline 3.60 & 73985.8 & 0.309 & 3.60 & 84764.9 & 0.029 & 3.60 & 93980.7 & 0.058 \\
\hline 3.63 & 74015.2 & 0.311 & 3.63 & 84757.0 & 0.028 & 3.63 & 94004.8 & 0.056 \\
\hline 3.66 & 74044.0 & 0.313 & 3.66 & 84748.3 & 0.026 & 3.66 & 94028.0 & 0.053 \\
\hline 3.69 & 74072.3 & 0.314 & 3.69 & 84738.9 & 0.026 & 3.69 & 94050.4 & 0.051 \\
\hline 3.72 & 74100.0 & 0.316 & 3.72 & 84728.8 & 0.025 & 3.72 & 94071.9 & 0.049 \\
\hline 3.75 & 74127.2 & 0.317 & 3.75 & 84718.2 & 0.026 & 3.75 & 94092.6 & 0.047 \\
\hline 3.78 & 74153.9 & 0.319 & 3.78 & 84707.1 & 0.026 & 3.78 & 94112.5 & 0.046 \\
\hline 3.81 & 74180.0 & 0.320 & 3.81 & 84695.5 & 0.028 & 3.81 & 94131.7 & 0.044 \\
\hline 3.84 & 74205.6 & 0.321 & 3.84 & 84683.6 & 0.029 & 3.84 & 94150.2 & 0.042 \\
\hline 3.87 & 74230.7 & 0.322 & 3.87 & 84671.3 & 0.030 & 3.87 & 94168.1 & 0.041 \\
\hline 3.90 & 74255.2 & 0.324 & 3.90 & 84658.8 & 0.031 & 3.90 & 94185.3 & 0.039 \\
\hline 3.93 & 74279.1 & 0.324 & 3.93 & 84646.1 & 0.031 & 3.93 & 94201.9 & 0.038 \\
\hline 3.96 & 74302.5 & 0.325 & 3.96 & 84633.1 & 0.030 & 3.96 & 94218.0 & 0.036 \\
\hline 3.99 & 74325.4 & 0.326 & 3.99 & 84620.0 & 0.028 & 3.99 & 94233.5 & 0.035 \\
\hline 4.00 & 74332.4 & 0.326 & 4.02 & 84606.9 & 0.026 & 4.02 & 94248.5 & 0.034 \\
\hline 4.03 & 74355.0 & 0.327 & 4.05 & 84593.8 & 0.023 & 4.05 & 94263.0 & 0.032 \\
\hline 4.06 & 74376.6 & 0.327 & 4.08 & 84580.6 & 0.019 & 4.08 & 94277.0 & 0.031 \\
\hline 4.09 & 74397.7 & 0.327 & 4.11 & 84568.4 & 0.015 & 4.11 & 94290.6 & 0.030 \\
\hline 4.12 & 74418.2 & 0.327 & 4.14 & 84555.5 & 0.010 & 4.14 & 94303.7 & 0.029 \\
\hline 4.15 & 74438.1 & 0.327 & 4.17 & 84542.9 & 0.006 & 4.17 & 94316.4 & 0.028 \\
\hline 4.18 & 74457.5 & 0.327 & 4.20 & 84530.6 & 0.005 & 4.20 & 94328.7 & 0.027 \\
\hline 4.21 & 74476.4 & 0.326 & 4.23 & 84518.7 & 0.005 & 4.23 & 94340.7 & 0.026 \\
\hline 4.24 & 74494.6 & 0.326 & 4.26 & 84507.1 & 0.004 & 4.26 & 94352.2 & 0.025 \\
\hline 4.27 & 74512.6 & 0.325 & 4.29 & 84496.1 & 0.003 & 4.29 & 94363.4 & 0.024 \\
\hline 4.30 & 74529.6 & 0.325 & 4.32 & 84485.6 & 0.002 & 4.32 & 94374.3 & 0.023 \\
\hline 4.33 & 74546.4 & 0.324 & 4.35 & 84475.7 & 0.002 & 4.35 & 94384.8 & 0.022 \\
\hline 4.36 & 74562.5 & 0.323 & 4.38 & 84466.6 & 0.001 & 4.38 & 94395.0 & 0.021 \\
\hline 4.39 & 74578.1 & 0.322 & 4.41 & 84458.4 & 0.001 & 4.41 & 94404.8 & 0.020 \\
\hline 4.42 & 74593.1 & 0.320 & 4.44 & 84451.3 & 0.000 & 4.44 & 94414.4 & 0.020 \\
\hline
\end{tabular}




\begin{tabular}{|c|c|c|c|c|c|c|c|c|}
\hline 4.45 & 74607.5 & 0.319 & 4.47 & 84445.5 & 0.000 & 4.48 & 94423.6 & 0.019 \\
\hline 4.48 & 74621.4 & 0.317 & & & & & & \\
\hline
\end{tabular}

\begin{tabular}{|c|c|c|c|c|c|c|c|c|}
\hline \multicolumn{3}{|c|}{$(1)^{4} \Delta$} & \multicolumn{3}{|c|}{$(2)^{4} \Delta$} & \multicolumn{3}{|c|}{$(1)^{4} \Sigma^{-}$} \\
\hline $\mathrm{R}(\AA)$ & $\mathrm{E}\left(\mathrm{cm}^{-1}\right)$ & $\mu$ (a.u.) & $\mathrm{R}(\AA)$ & $\mathrm{E}\left(\mathrm{cm}^{-1}\right)$ & $\mu$ (a.u.) & $\mathrm{R}(\AA)$ & $\mathrm{E}\left(\mathrm{cm}^{-1}\right)$ & $\mu$ (a.u.) \\
\hline 1.41 & 107486.6 & 1.078 & 1.41 & 154184.1 & -0.897 & 1.41 & 108952.3 & 1.108 \\
\hline 1.44 & 100335.8 & 1.066 & 1.44 & 147115.7 & -0.828 & 1.44 & 101767.6 & 1.096 \\
\hline 1.47 & 94091.0 & 1.052 & 1.47 & 140978.7 & -0.762 & 1.47 & 95487.9 & 1.082 \\
\hline 1.50 & 88634.2 & 1.037 & 1.50 & 135647.4 & -0.698 & 1.50 & 89995.5 & 1.067 \\
\hline 1.53 & 83862.7 & 1.020 & 1.53 & 131013.0 & -0.635 & 1.53 & 85188.0 & 1.050 \\
\hline 1.56 & 79687.2 & 1.003 & 1.56 & 126981.3 & -0.572 & 1.56 & 80976.1 & 1.032 \\
\hline 1.59 & 76030.3 & 0.984 & 1.59 & 123470.7 & -0.510 & 1.59 & 77282.8 & 1.013 \\
\hline 1.62 & 72824.9 & 0.965 & 1.62 & 120411.0 & -0.449 & 1.62 & 74041.0 & 0.994 \\
\hline 1.65 & 70013.0 & 0.946 & 1.65 & 117741.4 & -0.388 & 1.65 & 71192.7 & 0.974 \\
\hline 1.68 & 67544.6 & 0.926 & 1.68 & 115409.7 & -0.327 & 1.68 & 68688.0 & 0.954 \\
\hline 1.71 & 65376.2 & 0.906 & 1.71 & 113370.8 & -0.266 & 1.71 & 66483.5 & 0.933 \\
\hline 1.74 & 63470.5 & 0.886 & 1.74 & 111585.8 & -0.207 & 1.74 & 64541.9 & 0.912 \\
\hline 1.77 & 61795.0 & 0.865 & 1.77 & 110021.0 & -0.148 & 1.77 & 62830.9 & 0.891 \\
\hline 1.80 & 60321.5 & 0.845 & 1.80 & 108647.6 & -0.091 & 1.80 & 61322.3 & 0.870 \\
\hline 1.83 & 59025.4 & 0.824 & 1.83 & 107440.3 & -0.035 & 1.83 & 59991.8 & 0.848 \\
\hline 1.86 & 57885.4 & 0.802 & 1.86 & 106377.4 & 0.020 & 1.86 & 58818.1 & 0.826 \\
\hline 1.89 & 56882.8 & 0.781 & 1.89 & 105439.9 & 0.072 & 1.89 & 57782.4 & 0.805 \\
\hline 1.92 & 56001.1 & 0.760 & 1.92 & 104611.1 & 0.121 & 1.92 & 56868.4 & 0.783 \\
\hline 1.95 & 55225.9 & 0.739 & 1.95 & 103876.7 & 0.168 & 1.95 & 56061.9 & 0.761 \\
\hline 1.98 & 54544.6 & 0.717 & 1.98 & 103224.2 & 0.213 & 1.98 & 55350.1 & 0.738 \\
\hline 2.01 & 53946.0 & 0.696 & 2.01 & 102642.5 & 0.254 & 2.01 & 54721.7 & 0.716 \\
\hline 2.04 & 53420.2 & 0.674 & 2.04 & 102122.1 & 0.292 & 2.04 & 54167.0 & 0.694 \\
\hline 2.07 & 52958.5 & 0.653 & 2.07 & 101654.8 & 0.326 & 2.07 & 53677.2 & 0.672 \\
\hline 2.10 & 52553.1 & 0.632 & 2.10 & 101233.5 & 0.358 & 2.10 & 53244.5 & 0.651 \\
\hline 2.13 & 52197.3 & 0.611 & 2.13 & 100851.9 & 0.386 & 2.13 & 52862.3 & 0.629 \\
\hline 2.16 & 51885.0 & 0.590 & 2.16 & 100504.8 & 0.411 & 2.16 & 52524.4 & 0.608 \\
\hline 2.19 & 51610.9 & 0.570 & 2.19 & 100187.6 & 0.433 & 2.19 & 52225.5 & 0.586 \\
\hline 2.22 & 51370.5 & 0.550 & 2.22 & 99896.5 & 0.452 & 2.22 & 51961.1 & 0.566 \\
\hline 2.25 & 51159.6 & 0.530 & 2.25 & 99627.9 & 0.468 & 2.25 & 51727.0 & 0.545 \\
\hline 2.28 & 50974.6 & 0.510 & 2.28 & 99379.2 & 0.481 & 2.28 & 51519.6 & 0.525 \\
\hline 2.31 & 50812.4 & 0.491 & 2.31 & 99147.9 & 0.492 & 2.31 & 51335.9 & 0.505 \\
\hline 2.34 & 50670.3 & 0.472 & 2.34 & 98931.9 & 0.500 & 2.34 & 51173.0 & 0.486 \\
\hline 2.37 & 50545.8 & 0.454 & 2.37 & 98729.5 & 0.506 & 2.37 & 51028.4 & 0.467 \\
\hline 2.40 & 50436.9 & 0.436 & 2.40 & 98539.0 & 0.510 & 2.40 & 50900.3 & 0.449 \\
\hline 2.43 & 50341.8 & 0.419 & 2.43 & 98359.3 & 0.513 & 2.43 & 50786.5 & 0.431 \\
\hline 2.46 & 50258.7 & 0.402 & 2.46 & 98189.2 & 0.513 & 2.46 & 50685.6 & 0.414 \\
\hline 2.49 & 50186.4 & 0.386 & 2.49 & 98027.9 & 0.512 & 2.49 & 50596.1 & 0.397 \\
\hline 2.52 & 50123.5 & 0.370 & 2.52 & 97874.5 & 0.510 & 2.52 & 50516.7 & 0.381 \\
\hline 2.55 & 50069.1 & 0.355 & 2.55 & 97728.4 & 0.506 & 2.55 & 50446.4 & 0.365 \\
\hline
\end{tabular}




\begin{tabular}{|c|c|c|c|c|c|c|c|c|}
\hline 2.58 & 50022.0 & 0.340 & 2.58 & 97589.0 & 0.502 & 2.58 & 50384.2 & 0.350 \\
\hline 2.61 & 49981.5 & 0.325 & 2.61 & 97455.9 & 0.496 & 2.61 & 50329.1 & 0.335 \\
\hline 2.64 & 49946.9 & 0.312 & 2.64 & 97328.7 & 0.489 & 2.64 & 50280.5 & 0.321 \\
\hline 2.67 & 49917.4 & 0.298 & 2.67 & 97206.9 & 0.482 & 2.67 & 50237.7 & 0.307 \\
\hline 2.70 & 49892.6 & 0.285 & 2.70 & 97090.2 & 0.474 & 2.70 & 50200.1 & 0.294 \\
\hline 2.73 & 49871.9 & 0.273 & 2.73 & 96978.2 & 0.466 & 2.73 & 50167.1 & 0.282 \\
\hline 2.76 & 49854.9 & 0.261 & 2.76 & 96870.9 & 0.457 & 2.76 & 50138.3 & 0.270 \\
\hline 2.79 & 49841.2 & 0.250 & 2.79 & 96767.9 & 0.448 & 2.79 & 50113.3 & 0.258 \\
\hline 2.82 & 49830.3 & 0.239 & 2.82 & 96669.1 & 0.438 & 2.82 & 50091.7 & 0.247 \\
\hline 2.86 & 49822.2 & 0.228 & 2.85 & 96574.2 & 0.428 & 2.85 & 50073.2 & 0.236 \\
\hline 2.88 & 49820.0 & 0.218 & 2.88 & 96479.0 & 0.416 & 2.87 & 50063.7 & 0.225 \\
\hline 2.90 & 49818.5 & 0.208 & 2.91 & 96391.6 & 0.406 & 2.91 & 50050.5 & 0.215 \\
\hline 2.94 & 49816.7 & 0.199 & 2.94 & 96307.8 & 0.396 & 2.94 & 50043.0 & 0.204 \\
\hline 2.97 & 49816.5 & 0.190 & 2.97 & 96227.4 & 0.385 & 2.97 & 50034.0 & 0.195 \\
\hline 3.00 & 49817.8 & 0.182 & 3.00 & 96150.2 & 0.375 & 3.00 & 50026.8 & 0.187 \\
\hline 3.03 & 49820.4 & 0.174 & 3.03 & 96076.3 & 0.365 & 3.03 & 50021.4 & 0.178 \\
\hline 3.06 & 49824.3 & 0.166 & 3.06 & 96005.4 & 0.354 & 3.06 & 50017.5 & 0.170 \\
\hline 3.09 & 49829.3 & 0.159 & 3.09 & 95937.5 & 0.344 & 3.09 & 50015.3 & 0.163 \\
\hline 3.12 & 49835.3 & 0.152 & 3.12 & 95872.5 & 0.334 & 3.12 & 50014.1 & 0.156 \\
\hline 3.15 & 49842.1 & 0.145 & 3.15 & 95809.6 & 0.324 & 3.15 & 50013.9 & 0.149 \\
\hline 3.18 & 49849.5 & 0.138 & 3.18 & 95742.8 & 0.313 & 3.18 & 50014.2 & 0.142 \\
\hline 3.21 & 49857.8 & 0.132 & 3.21 & 95685.3 & 0.304 & 3.21 & 50016.2 & 0.136 \\
\hline 3.24 & 49866.7 & 0.126 & 3.24 & 95630.1 & 0.294 & 3.24 & 50019.2 & 0.130 \\
\hline 3.27 & 49876.0 & 0.121 & 3.27 & 95577.6 & 0.284 & 3.27 & 50022.9 & 0.124 \\
\hline 3.30 & 49885.8 & 0.116 & 3.30 & 95527.4 & 0.275 & 3.30 & 50027.2 & 0.119 \\
\hline 3.33 & 49896.0 & 0.111 & 3.33 & 95479.4 & 0.266 & 3.33 & 50032.2 & 0.114 \\
\hline 3.36 & 49906.5 & 0.106 & 3.36 & 95433.6 & 0.257 & 3.36 & 50037.7 & 0.109 \\
\hline 3.39 & 49917.3 & 0.101 & 3.39 & 95389.9 & 0.248 & 3.39 & 50043.7 & 0.104 \\
\hline 3.42 & 49928.2 & 0.097 & 3.42 & 95348.1 & 0.240 & 3.42 & 50050.1 & 0.099 \\
\hline 3.45 & 49939.3 & 0.093 & 3.45 & 95308.3 & 0.231 & 3.45 & 50056.8 & 0.095 \\
\hline 3.48 & 49950.4 & 0.089 & 3.48 & 95270.3 & 0.223 & 3.48 & 50063.8 & 0.091 \\
\hline 3.51 & 49961.7 & 0.085 & 3.51 & 95234.0 & 0.215 & 3.51 & 50071.0 & 0.087 \\
\hline 3.54 & 49972.9 & 0.081 & 3.54 & 95199.7 & 0.207 & 3.54 & 50078.4 & 0.083 \\
\hline 3.57 & 49984.4 & 0.078 & 3.57 & 95166.8 & 0.200 & 3.57 & 50086.0 & 0.080 \\
\hline 3.60 & 49995.6 & 0.075 & 3.60 & 95135.5 & 0.192 & 3.60 & 50093.7 & 0.077 \\
\hline 3.63 & 50006.8 & 0.071 & 3.63 & 95105.7 & 0.185 & 3.63 & 50101.5 & 0.073 \\
\hline 3.66 & 50017.9 & 0.068 & 3.66 & 95077.4 & 0.178 & 3.66 & 50109.4 & 0.070 \\
\hline 3.69 & 50029.0 & 0.066 & 3.69 & 95050.5 & 0.172 & 3.69 & 50117.3 & 0.067 \\
\hline 3.72 & 50039.9 & 0.063 & 3.72 & 95025.0 & 0.165 & 3.72 & 50125.2 & 0.065 \\
\hline 3.75 & 50050.7 & 0.060 & 3.75 & 95000.7 & 0.159 & 3.75 & 50133.2 & 0.062 \\
\hline 3.78 & 50061.3 & 0.058 & 3.78 & 94977.8 & 0.153 & 3.78 & 50141.1 & 0.059 \\
\hline 3.81 & 50071.9 & 0.055 & 3.81 & 94956.0 & 0.147 & 3.81 & 50149.0 & 0.057 \\
\hline 3.84 & 50082.3 & 0.053 & 3.84 & 94935.4 & 0.141 & 3.84 & 50156.9 & 0.055 \\
\hline 3.87 & 50092.6 & 0.051 & 3.87 & 94914.7 & 0.135 & 3.87 & 50164.8 & 0.052 \\
\hline
\end{tabular}




\begin{tabular}{|c|c|c|c|c|c|c|c|c|}
\hline 3.90 & 50102.7 & 0.049 & 3.90 & 94896.3 & 0.130 & 3.90 & 50172.6 & 0.050 \\
\hline 3.93 & 50112.6 & 0.047 & 3.93 & 94878.9 & 0.125 & 3.93 & 50180.4 & 0.048 \\
\hline 3.96 & 50123.1 & 0.045 & 3.96 & 94862.6 & 0.120 & 3.96 & 50188.0 & 0.046 \\
\hline 3.99 & 50132.8 & 0.043 & 3.99 & 94848.4 & 0.115 & 4.00 & 50199.5 & 0.044 \\
\hline 4.00 & 50136.5 & 0.043 & 4.02 & 94833.9 & 0.110 & 4.03 & 50205.8 & 0.042 \\
\hline 4.03 & 50145.4 & 0.041 & 4.05 & 94818.9 & 0.106 & 4.06 & 50213.3 & 0.040 \\
\hline 4.06 & 50154.7 & 0.039 & 4.08 & 94806.0 & 0.101 & 4.09 & 50220.6 & 0.039 \\
\hline 4.09 & 50163.8 & 0.038 & 4.11 & 94794.0 & 0.097 & 4.12 & 50227.9 & 0.037 \\
\hline 4.12 & 50172.8 & 0.036 & 4.14 & 94782.8 & 0.093 & 4.15 & 50235.1 & 0.036 \\
\hline 4.15 & 50181.7 & 0.035 & 4.17 & 94772.1 & 0.089 & 4.18 & 50242.5 & 0.034 \\
\hline 4.18 & 50190.4 & 0.033 & 4.20 & 94762.3 & 0.086 & 4.21 & 50249.5 & 0.033 \\
\hline 4.21 & 50198.9 & 0.032 & 4.23 & 94753.1 & 0.082 & 4.24 & 50256.4 & 0.032 \\
\hline 4.24 & 50207.2 & 0.031 & 4.26 & 94744.6 & 0.079 & 4.27 & 50263.2 & 0.030 \\
\hline 4.27 & 50215.4 & 0.030 & 4.29 & 94736.8 & 0.075 & 4.30 & 50269.8 & 0.029 \\
\hline 4.30 & 50223.4 & 0.028 & 4.32 & 94731.1 & 0.072 & 4.33 & 50276.4 & 0.028 \\
\hline 4.33 & 50231.3 & 0.027 & 4.35 & 94724.4 & 0.069 & 4.36 & 50282.9 & 0.027 \\
\hline 4.36 & 50239.0 & 0.026 & 4.48 & 94718.2 & 0.066 & 4.39 & 50289.2 & 0.026 \\
\hline 4.39 & 50246.5 & 0.025 & & & & 4.42 & 50295.4 & 0.025 \\
\hline 4.42 & 50253.9 & 0.024 & & & & 4.45 & 50301.5 & 0.024 \\
\hline 4.45 & 50261.1 & 0.023 & & & & 4.48 & 50307.4 & 0.023 \\
\hline 4.48 & 50268.2 & 0.022 & & & & & & \\
\hline
\end{tabular}

\begin{tabular}{|c|c|c|}
\hline \multicolumn{3}{|c|}{$(2)^{4} \Sigma^{-}$} \\
\hline $\mathrm{R}(\AA)$ & $\mathrm{E}\left(\mathrm{cm}^{-1}\right)$ & $\mu$ (a.u.) \\
\hline 1.41 & 146056.0 & 0.420 \\
\hline 1.44 & 139664.5 & 0.427 \\
\hline 1.47 & 134340.4 & 0.435 \\
\hline 1.50 & 129667.9 & 0.442 \\
\hline 1.53 & 125877.3 & 0.448 \\
\hline 1.56 & 122314.4 & 0.449 \\
\hline 1.59 & 119008.6 & 0.450 \\
\hline 1.62 & 115680.1 & 0.448 \\
\hline 1.65 & 113243.9 & 0.443 \\
\hline 1.68 & 111312.6 & 0.439 \\
\hline 1.71 & 109740.6 & 0.433 \\
\hline 1.74 & 108249.9 & 0.422 \\
\hline 1.77 & 106681.6 & 0.412 \\
\hline 1.79 & 105595.0 & 0.403 \\
\hline 1.82 & 104445.0 & 0.393 \\
\hline 1.84 & 103694.6 & 0.387 \\
\hline 1.88 & 102401.9 & 0.372 \\
\hline 1.90 & 101787.4 & 0.367 \\
\hline 1.91 & 101458.1 & 0.361 \\
\hline 1.97 & 99829.6 & 0.341 \\
\hline 2.01 & 99098.8 & 0.327 \\
\hline
\end{tabular}




\begin{tabular}{|c|c|c|}
\hline 2.04 & 98736.7 & 0.316 \\
\hline 2.06 & 98385.5 & 0.308 \\
\hline 2.10 & 97946.5 & 0.299 \\
\hline 2.13 & 97705.1 & 0.292 \\
\hline 2.16 & 97441.8 & 0.285 \\
\hline 2.17 & 97375.9 & 0.282 \\
\hline 2.21 & 97068.6 & 0.274 \\
\hline 2.24 & 96890.3 & 0.268 \\
\hline 2.27 & 96706.5 & 0.259 \\
\hline 2.31 & 96533.1 & 0.250 \\
\hline 2.34 & 96410.2 & 0.240 \\
\hline 2.37 & 96322.4 & 0.232 \\
\hline 2.40 & 96212.7 & 0.225 \\
\hline 2.43 & 96138.1 & 0.218 \\
\hline 2.46 & 96054.7 & 0.212 \\
\hline 2.49 & 95968.4 & 0.209 \\
\hline 2.52 & 95890.1 & 0.204 \\
\hline 2.55 & 95828.3 & 0.201 \\
\hline 2.58 & 95775.8 & 0.200 \\
\hline 2.61 & 95736.9 & 0.202 \\
\hline 2.64 & 95708.1 & 0.203 \\
\hline 2.67 & 95688.6 & 0.202 \\
\hline 2.70 & 95677.7 & 0.205 \\
\hline 2.73 & 95675.0 & 0.209 \\
\hline 2.76 & 95677.9 & 0.214 \\
\hline 2.79 & 95685.6 & 0.219 \\
\hline 2.82 & 95695.6 & 0.225 \\
\hline 2.85 & 95706.6 & 0.233 \\
\hline 2.88 & 95683.1 & 0.235 \\
\hline 2.91 & 95697.4 & 0.237 \\
\hline 2.94 & 95097.1 & 0.237 \\
\hline 2.97 & 95073.0 & 0.233 \\
\hline 3.00 & 95050.6 & 0.229 \\
\hline 3.03 & 95029.7 & 0.226 \\
\hline 3.06 & 95010.3 & 0.223 \\
\hline 3.09 & 94992.2 & 0.220 \\
\hline 3.12 & 94975.2 & 0.217 \\
\hline 3.15 & 94958.8 & 0.214 \\
\hline 3.18 & 94945.9 & 0.211 \\
\hline 3.21 & 94931.8 & 0.209 \\
\hline 3.24 & 94918.6 & 0.207 \\
\hline 3.27 & 94906.2 & 0.204 \\
\hline 3.30 & 94894.5 & 0.203 \\
\hline 3.33 & 94883.5 & 0.201 \\
\hline
\end{tabular}




\begin{tabular}{|c|c|c|}
\hline 3.36 & 94873.2 & 0.199 \\
\hline 3.39 & 94863.4 & 0.198 \\
\hline 3.42 & 94854.2 & 0.196 \\
\hline 3.45 & 94845.5 & 0.195 \\
\hline 3.48 & 94837.2 & 0.194 \\
\hline 3.51 & 94829.3 & 0.193 \\
\hline 3.54 & 94821.8 & 0.192 \\
\hline 3.57 & 94814.7 & 0.192 \\
\hline 3.60 & 94807.8 & 0.191 \\
\hline 3.63 & 94801.3 & 0.190 \\
\hline 3.66 & 94795.0 & 0.190 \\
\hline 3.69 & 94788.9 & 0.190 \\
\hline 3.72 & 94783.1 & 0.189 \\
\hline 3.75 & 94777.5 & 0.189 \\
\hline 3.78 & 94772.1 & 0.189 \\
\hline 3.81 & 94766.8 & 0.189 \\
\hline 3.84 & 94761.7 & 0.189 \\
\hline 3.87 & 94756.8 & 0.189 \\
\hline 3.90 & 94752.0 & 0.189 \\
\hline 3.93 & 94747.3 & 0.189 \\
\hline 3.96 & 94742.8 & 0.189 \\
\hline 3.99 & 94738.5 & 0.189 \\
\hline 4.02 & 94734.2 & 0.189 \\
\hline 4.05 & 94730.1 & 0.189 \\
\hline 4.08 & 94726.2 & 0.189 \\
\hline 4.11 & 94722.3 & 0.188 \\
\hline 4.14 & 94718.6 & 0.188 \\
\hline 4.17 & 94715.1 & 0.188 \\
\hline 4.20 & 94711.7 & 0.187 \\
\hline 4.23 & 94708.4 & 0.187 \\
\hline 4.26 & 94705.3 & 0.186 \\
\hline 4.29 & 94702.4 & 0.185 \\
\hline 4.32 & 94699.5 & 0.185 \\
\hline 4.35 & 94697.5 & 0.184 \\
\hline 4.38 & 94695.0 & 0.183 \\
\hline 4.41 & 94692.6 & 0.182 \\
\hline 4.44 & 94690.4 & 0.181 \\
\hline 4.48 & 94688.4 & 0.180 \\
\hline
\end{tabular}


Table (SM.2): Numerical data corresponding to the transition dipole moment (a.u.) some low-lying doublet states:

\begin{tabular}{|c|c|c|c|c|c|c|}
\hline \multirow[b]{2}{*}{$\mathrm{R}(\AA)$} & \multicolumn{6}{|c|}{ Transition dipole moment (a.u.) } \\
\hline & $\mathrm{X}^{2} \Sigma^{+}-(1)^{2} \Pi$ & $\mathrm{X}^{2} \Sigma^{+}-(2)^{2} \Sigma^{+}$ & $(1)^{2} \Pi-(1)^{2} \Delta$ & $(2)^{2} \Pi-(1)^{2} \Delta$ & $(1)^{2} \Pi-(1)^{2} \Sigma^{-}$ & $(2)^{2} \Pi-(1)^{2} \Sigma^{-}$ \\
\hline 1.40 & 1.466 & 1.042 & -0.247 & 0.009 & -0.280 & 0.095 \\
\hline 1.43 & 1.465 & 1.044 & -0.248 & 0.013 & -0.279 & 0.091 \\
\hline 1.46 & 1.461 & 1.045 & -0.249 & 0.015 & -0.278 & 0.088 \\
\hline 1.49 & 1.454 & 1.044 & -0.250 & 0.017 & -0.275 & 0.085 \\
\hline 1.52 & 1.446 & 1.036 & -0.249 & 0.017 & -0.272 & 0.083 \\
\hline 1.55 & 1.435 & 1.018 & -0.249 & 0.017 & -0.269 & 0.080 \\
\hline 1.58 & 1.420 & 0.970 & -0.248 & 0.018 & -0.265 & 0.078 \\
\hline 1.61 & 1.400 & 0.836 & -0.246 & 0.015 & -0.260 & 0.073 \\
\hline 1.64 & 1.372 & 0.568 & -0.244 & 0.014 & -0.253 & 0.072 \\
\hline 1.67 & 1.329 & 0.276 & -0.240 & 0.005 & -0.244 & 0.060 \\
\hline 1.70 & 1.261 & 0.062 & -0.233 & -0.245 & -0.231 & -0.260 \\
\hline 1.73 & 1.148 & -0.077 & -0.220 & -0.243 & -0.210 & -0.254 \\
\hline 1.76 & 0.960 & -0.170 & -0.196 & -0.240 & -0.175 & -0.248 \\
\hline 1.79 & 0.694 & -0.239 & -0.158 & -0.235 & -0.127 & -0.239 \\
\hline 1.82 & 0.431 & -0.292 & -0.118 & -0.228 & -0.080 & -0.226 \\
\hline 1.85 & 0.240 & -0.337 & -0.088 & -0.211 & -0.046 & -0.198 \\
\hline 1.88 & 0.116 & -0.377 & -0.068 & 0.142 & -0.024 & 0.185 \\
\hline 1.91 & 0.035 & -0.413 & -0.055 & -0.202 & -0.010 & -0.230 \\
\hline 1.94 & -0.019 & -0.448 & -0.045 & -0.218 & -0.001 & -0.232 \\
\hline 1.97 & -0.058 & -0.482 & -0.039 & -0.210 & 0.005 & -0.226 \\
\hline 2.00 & -0.086 & -0.516 & -0.034 & -0.207 & 0.009 & -0.218 \\
\hline 2.03 & -0.106 & -0.550 & -0.030 & -0.204 & 0.012 & -0.215 \\
\hline 2.06 & -0.121 & -0.585 & -0.027 & -0.199 & 0.014 & -0.209 \\
\hline 2.09 & -0.133 & -0.620 & -0.025 & -0.194 & 0.015 & -0.202 \\
\hline 2.12 & -0.142 & -0.657 & -0.022 & -0.189 & 0.016 & -0.196 \\
\hline 2.15 & -0.149 & -0.695 & -0.021 & -0.183 & 0.016 & -0.189 \\
\hline 2.18 & -0.154 & -0.734 & -0.019 & -0.177 & 0.016 & -0.182 \\
\hline 2.21 & -0.157 & -0.774 & -0.018 & -0.171 & 0.016 & -0.175 \\
\hline 2.24 & -0.160 & -0.816 & -0.017 & -0.165 & 0.016 & -0.168 \\
\hline 2.27 & -0.161 & -0.860 & -0.015 & -0.158 & 0.016 & -0.161 \\
\hline 2.30 & -0.162 & -0.904 & -0.014 & -0.151 & 0.015 & -0.154 \\
\hline 2.33 & -0.162 & -0.949 & -0.013 & -0.145 & 0.015 & -0.147 \\
\hline 2.36 & -0.161 & -0.994 & -0.013 & -0.138 & 0.014 & -0.140 \\
\hline 2.39 & -0.160 & -1.040 & -0.012 & -0.131 & 0.014 & -0.133 \\
\hline 2.42 & -0.158 & -1.085 & -0.011 & -0.124 & 0.013 & -0.126 \\
\hline 2.45 & -0.155 & -1.128 & -0.010 & -0.118 & 0.013 & -0.119 \\
\hline 2.48 & -0.152 & -1.169 & -0.010 & -0.111 & 0.012 & -0.112 \\
\hline 2.51 & -0.149 & -1.208 & -0.009 & -0.105 & 0.011 & -0.106 \\
\hline 2.54 & -0.145 & -1.242 & -0.008 & -0.099 & 0.011 & -0.099 \\
\hline 2.57 & -0.141 & -1.272 & -0.008 & -0.093 & 0.010 & -0.094 \\
\hline 2.60 & -0.136 & -1.296 & -0.007 & -0.087 & 0.010 & -0.088 \\
\hline 2.63 & -0.131 & -1.314 & -0.007 & -0.082 & 0.009 & -0.083 \\
\hline 2.66 & 0.126 & 1.325 & -0.006 & -0.077 & 0.008 & -0.077 \\
\hline 2.69 & 0.120 & 1.330 & -0.006 & -0.072 & 0.008 & -0.073 \\
\hline 2.72 & 0.114 & 1.328 & -0.005 & -0.067 & 0.007 & -0.068 \\
\hline 2.75 & 0.109 & 1.320 & -0.005 & -0.063 & 0.007 & -0.064 \\
\hline 2.78 & 0.103 & 1.307 & -0.004 & -0.059 & 0.006 & -0.060 \\
\hline
\end{tabular}




\begin{tabular}{|c|c|c|c|c|c|c|}
\hline 2.81 & 0.098 & 1.290 & -0.004 & -0.055 & 0.006 & -0.056 \\
\hline 2.84 & 0.092 & 1.268 & -0.004 & -0.051 & 0.005 & -0.053 \\
\hline 2.87 & 0.087 & 1.242 & -0.003 & -0.048 & 0.005 & -0.050 \\
\hline 2.90 & 0.082 & 1.214 & -0.003 & -0.045 & 0.005 & -0.047 \\
\hline 2.93 & 0.078 & 1.184 & -0.003 & -0.041 & 0.004 & -0.044 \\
\hline 2.96 & 0.074 & 1.152 & -0.003 & -0.038 & 0.004 & -0.042 \\
\hline 2.99 & 0.069 & 1.119 & -0.002 & -0.035 & 0.004 & -0.040 \\
\hline 3.02 & 0.066 & 1.086 & -0.002 & -0.032 & 0.003 & -0.038 \\
\hline 3.05 & 0.062 & 1.052 & -0.002 & -0.027 & 0.003 & -0.037 \\
\hline 3.08 & 0.059 & 1.019 & -0.002 & -0.022 & 0.003 & -0.035 \\
\hline 3.11 & 0.055 & 0.986 & -0.002 & -0.015 & 0.002 & -0.032 \\
\hline 3.14 & 0.053 & 0.953 & -0.001 & -0.009 & 0.002 & -0.029 \\
\hline 3.17 & 0.050 & 0.921 & -0.001 & -0.004 & 0.002 & -0.026 \\
\hline 3.20 & 0.047 & 0.890 & -0.001 & 0.000 & 0.002 & -0.024 \\
\hline 3.23 & 0.045 & 0.860 & -0.001 & 0.003 & 0.002 & -0.023 \\
\hline 3.26 & 0.042 & 0.830 & -0.001 & 0.005 & 0.001 & -0.022 \\
\hline 3.29 & 0.040 & 0.802 & -0.001 & 0.007 & 0.001 & -0.021 \\
\hline 3.32 & 0.038 & 0.774 & -0.001 & 0.009 & 0.001 & -0.020 \\
\hline 3.35 & 0.036 & 0.746 & -0.001 & 0.010 & 0.001 & -0.020 \\
\hline 3.38 & 0.035 & 0.720 & -0.001 & 0.011 & 0.001 & -0.020 \\
\hline 3.41 & 0.033 & 0.694 & -0.001 & 0.012 & 0.001 & -0.020 \\
\hline 3.44 & 0.032 & 0.670 & 0.000 & 0.012 & 0.001 & -0.019 \\
\hline 3.47 & 0.030 & 0.645 & 0.000 & 0.013 & 0.001 & -0.019 \\
\hline 3.50 & 0.029 & 0.622 & 0.000 & 0.013 & 0.000 & -0.019 \\
\hline 3.53 & 0.027 & 0.599 & 0.000 & 0.013 & 0.000 & -0.019 \\
\hline 3.56 & 0.026 & 0.576 & 0.000 & 0.014 & 0.000 & -0.018 \\
\hline 3.59 & 0.025 & 0.555 & 0.000 & 0.014 & 0.000 & -0.018 \\
\hline 3.62 & 0.024 & 0.533 & 0.000 & 0.014 & 0.000 & -0.018 \\
\hline 3.65 & 0.023 & 0.513 & 0.000 & 0.014 & 0.000 & -0.017 \\
\hline 3.68 & 0.022 & 0.492 & 0.000 & 0.014 & 0.000 & -0.017 \\
\hline 3.71 & 0.021 & 0.472 & 0.000 & 0.014 & 0.000 & -0.017 \\
\hline 3.74 & 0.020 & 0.453 & 0.000 & 0.013 & 0.000 & -0.016 \\
\hline 3.77 & 0.020 & 0.434 & 0.000 & 0.013 & 0.000 & -0.016 \\
\hline 3.80 & 0.019 & 0.415 & 0.000 & 0.013 & 0.000 & -0.016 \\
\hline 3.83 & 0.018 & 0.397 & 0.000 & 0.013 & 0.000 & -0.015 \\
\hline 3.86 & 0.017 & 0.379 & 0.000 & 0.013 & 0.000 & -0.015 \\
\hline 3.89 & 0.017 & 0.361 & 0.000 & 0.012 & 0.000 & -0.014 \\
\hline 3.92 & 0.016 & 0.344 & 0.000 & 0.012 & 0.000 & -0.014 \\
\hline 3.95 & 0.016 & 0.327 & 0.000 & 0.012 & 0.000 & -0.014 \\
\hline 3.98 & 0.015 & 0.310 & 0.000 & 0.011 & 0.000 & -0.013 \\
\hline 4.01 & 0.015 & 0.293 & 0.000 & 0.011 & 0.000 & -0.013 \\
\hline 4.04 & 0.014 & 0.277 & 0.000 & 0.011 & 0.000 & -0.012 \\
\hline 4.07 & 0.014 & 0.261 & 0.000 & 0.010 & 0.000 & -0.012 \\
\hline 4.10 & 0.013 & 0.245 & 0.000 & 0.010 & 0.000 & -0.011 \\
\hline 4.13 & 0.013 & 0.230 & 0.000 & 0.010 & 0.000 & -0.011 \\
\hline 4.16 & 0.012 & 0.215 & 0.000 & 0.009 & 0.000 & -0.011 \\
\hline 4.19 & 0.012 & 0.200 & 0.000 & 0.009 & 0.000 & -0.010 \\
\hline 4.22 & 0.012 & 0.186 & 0.000 & 0.009 & 0.000 & -0.010 \\
\hline 4.25 & 0.011 & -0.172 & 0.000 & 0.008 & 0.000 & -0.010 \\
\hline 4.28 & 0.011 & -0.159 & 0.000 & 0.008 & 0.000 & -0.009 \\
\hline 4.31 & 0.011 & -0.147 & 0.000 & 0.008 & 0.000 & -0.009 \\
\hline 4.34 & 0.010 & -0.135 & 0.000 & 0.007 & 0.000 & -0.008 \\
\hline
\end{tabular}




\begin{tabular}{|l|l|l|l|l|l|l|}
\hline 4.37 & 0.010 & -0.124 & 0.000 & 0.007 & 0.000 & -0.008 \\
\hline 4.40 & 0.010 & -0.113 & 0.000 & 0.007 & 0.000 & -0.008 \\
\hline 4.43 & 0.009 & -0.104 & 0.000 & 0.007 & 0.000 & -0.007 \\
\hline 4.46 & 0.009 & -0.094 & 0.000 & 0.006 & 0.000 & -0.007 \\
\hline 4.49 & 0.009 & -0.086 & 0.000 & 0.006 & 0.000 & -0.007 \\
\hline 4.52 & 0.009 & -0.078 & 0.000 & 0.006 & 0.000 & -0.007 \\
\hline 4.55 & 0.008 & -0.071 & 0.000 & 0.006 & 0.000 & -0.006 \\
\hline
\end{tabular}

\begin{tabular}{|c|c|c|c|c|c|}
\hline \multirow[b]{2}{*}{$\mathrm{R}(\AA)$} & \multicolumn{5}{|c|}{ Transition dipole moment (a.u.) } \\
\hline & $(1)^{2} \Pi-(2)^{2} \Pi$ & $X^{2} \Sigma^{+}-(2)^{2} \Pi$ & $X^{2} \Sigma^{+}-(3)^{2} \Sigma^{+}$ & $X^{2} \Sigma^{+}-(4)^{2} \Sigma^{+}$ & $(2)^{2} \Sigma^{+}-(3)^{2} \Sigma^{+}$ \\
\hline 1.40 & 0.206 & -0.395 & 0.010 & -0.447 & -0.713 \\
\hline 1.42 & 0.210 & -0.395 & -0.016 & -0.442 & -0.672 \\
\hline 1.43 & 0.215 & -0.399 & -0.050 & -0.444 & -0.595 \\
\hline 1.45 & 0.220 & -0.399 & -0.111 & -0.441 & -0.481 \\
\hline 1.48 & 0.229 & -0.400 & -0.197 & -0.427 & -0.299 \\
\hline 1.49 & 0.234 & -0.400 & -0.238 & -0.408 & -0.252 \\
\hline 1.52 & 0.247 & -0.400 & -0.224 & -0.360 & -0.247 \\
\hline 1.54 & 0.253 & -0.400 & -0.182 & -0.334 & -0.318 \\
\hline 1.55 & 0.261 & -0.397 & -0.061 & -0.284 & -0.456 \\
\hline 1.57 & 0.269 & -0.397 & 0.452 & 1.123 & -0.971 \\
\hline 1.58 & 0.276 & -0.397 & 1.137 & 0.196 & -1.695 \\
\hline 1.60 & 0.287 & -0.397 & 1.152 & -0.968 & -1.714 \\
\hline 1.61 & 0.300 & -0.386 & 1.153 & -0.957 & -1.689 \\
\hline 1.63 & 0.321 & -0.386 & 1.151 & -0.757 & -1.596 \\
\hline 1.64 & 0.342 & -0.377 & 1.143 & 0.038 & -1.442 \\
\hline 1.67 & 0.355 & -0.323 & 1.127 & 0.419 & -1.092 \\
\hline 1.68 & -1.482 & 0.162 & 1.121 & -0.952 & -0.978 \\
\hline 1.69 & -1.471 & 0.742 & 1.113 & -0.949 & -0.864 \\
\hline 1.70 & -1.455 & 1.426 & 1.101 & -0.824 & -0.743 \\
\hline 1.71 & -1.433 & 1.420 & 1.091 & -0.734 & -0.645 \\
\hline 1.72 & -1.404 & 1.413 & 1.077 & -0.636 & -0.544 \\
\hline 1.73 & -1.366 & 1.406 & 1.054 & -0.543 & -0.432 \\
\hline 1.74 & -1.317 & 1.398 & 1.034 & -0.472 & -0.309 \\
\hline 1.75 & -1.256 & 1.389 & 0.993 & -0.437 & -0.200 \\
\hline 1.76 & -1.181 & 1.379 & 0.942 & -0.342 & -0.087 \\
\hline 1.77 & -1.091 & 1.368 & 0.844 & -0.284 & 0.033 \\
\hline 1.78 & -0.988 & 1.356 & 0.723 & -0.254 & 0.128 \\
\hline 1.79 & -0.874 & 1.341 & 0.647 & -0.181 & 0.208 \\
\hline 1.80 & -0.753 & 1.323 & 0.654 & -0.135 & 0.220 \\
\hline 1.81 & -0.629 & 1.302 & 0.694 & -0.108 & 0.208 \\
\hline 1.82 & -0.503 & 1.275 & 0.767 & -0.032 & 0.146 \\
\hline 1.83 & -0.374 & 1.239 & 0.893 & 0.014 & -0.007 \\
\hline 1.84 & -0.239 & 1.190 & 1.054 & 0.035 & -0.402 \\
\hline 1.85 & -0.095 & 1.123 & 1.217 & 0.104 & -0.683 \\
\hline 1.86 & 0.061 & 1.047 & 1.221 & 0.152 & -0.695 \\
\hline 1.87 & 0.314 & 0.991 & 1.216 & 0.171 & -0.627 \\
\hline 1.88 & 1.044 & -1.067 & 1.208 & 0.240 & -0.554 \\
\hline 1.89 & -0.140 & 1.313 & 1.199 & 0.282 & -0.517 \\
\hline 1.90 & -0.331 & 1.367 & 1.186 & 0.308 & -0.492 \\
\hline 1.91 & -0.726 & 1.375 & 1.174 & -0.372 & -0.476 \\
\hline 1.92 & -0.587 & 1.374 & 1.156 & -0.435 & -0.445 \\
\hline
\end{tabular}




\begin{tabular}{|c|c|c|c|c|c|}
\hline 1.94 & -0.515 & 1.362 & 1.132 & -0.496 & -0.415 \\
\hline 1.96 & -0.435 & 1.347 & 1.099 & -0.557 & -0.385 \\
\hline 1.97 & -0.397 & 1.339 & 1.075 & -0.619 & -0.368 \\
\hline 1.98 & -0.362 & 1.330 & 1.052 & -0.652 & -0.354 \\
\hline 1.99 & -0.338 & 1.322 & 1.029 & -0.682 & -0.340 \\
\hline 2.00 & -0.322 & 1.315 & 1.000 & -0.746 & -0.326 \\
\hline 2.01 & -0.306 & 1.307 & 0.971 & -0.800 & -0.313 \\
\hline 2.02 & -0.294 & 1.300 & 0.944 & -0.847 & -0.301 \\
\hline 2.03 & -0.278 & 1.290 & 0.907 & -0.890 & -0.289 \\
\hline 2.05 & -0.263 & 1.277 & 0.864 & -0.971 & -0.266 \\
\hline 2.06 & -0.256 & 1.268 & 0.829 & -1.010 & -0.255 \\
\hline 2.07 & -0.248 & 1.259 & 0.802 & -1.053 & -0.244 \\
\hline 2.08 & -0.238 & 1.249 & 0.770 & -1.096 & -0.234 \\
\hline 2.09 & -0.231 & 1.240 & 0.736 & -1.139 & -0.225 \\
\hline 2.10 & -0.221 & 1.232 & 0.707 & -1.177 & -0.215 \\
\hline 2.11 & -0.212 & 1.221 & 0.676 & -1.197 & -0.207 \\
\hline 2.12 & -0.204 & 1.211 & 0.642 & -1.267 & -0.198 \\
\hline 2.13 & -0.196 & 1.200 & 0.614 & -1.306 & -0.190 \\
\hline 2.14 & -0.189 & 1.189 & 0.588 & -1.324 & -0.182 \\
\hline 2.15 & -0.182 & 1.179 & 0.553 & -1.373 & -0.175 \\
\hline 2.16 & -0.175 & 1.169 & 0.520 & -1.407 & -0.168 \\
\hline 2.18 & -0.163 & 1.148 & 0.470 & -1.435 & -0.156 \\
\hline 2.20 & -0.150 & 1.126 & 0.420 & 1.518 & -0.144 \\
\hline 2.21 & -0.145 & 1.114 & 0.395 & 1.569 & -0.139 \\
\hline 2.23 & -0.136 & 1.091 & 0.350 & 1.592 & -0.130 \\
\hline 2.24 & -0.132 & 1.079 & 0.328 & 1.606 & -0.122 \\
\hline 2.26 & -0.124 & 1.055 & 0.298 & 1.607 & -0.119 \\
\hline 2.27 & -0.120 & 1.042 & 0.269 & 1.257 & -0.116 \\
\hline 2.29 & -0.110 & 1.010 & 0.227 & -1.447 & -0.110 \\
\hline 2.31 & -0.110 & 0.990 & 0.195 & -1.482 & -0.105 \\
\hline 2.34 & -0.100 & 0.949 & 0.154 & -1.441 & -0.099 \\
\hline 2.36 & -0.089 & 0.919 & 0.133 & -1.390 & -0.096 \\
\hline 2.39 & -0.081 & 0.877 & 0.099 & -1.138 & -0.093 \\
\hline 2.41 & -0.075 & 0.849 & 0.080 & 1.280 & -0.091 \\
\hline 2.42 & -0.074 & 0.833 & 0.070 & 1.417 & -0.091 \\
\hline 2.44 & -0.068 & 0.801 & 0.052 & 1.523 & -0.090 \\
\hline 2.47 & -0.062 & 0.760 & 0.029 & 1.592 & -0.090 \\
\hline 2.48 & -0.060 & 0.743 & 0.023 & 1.604 & -0.090 \\
\hline 2.51 & -0.055 & 0.698 & 0.004 & 1.589 & -0.090 \\
\hline 2.54 & -0.050 & 0.653 & -0.011 & 1.569 & -0.092 \\
\hline 2.57 & -0.045 & 0.608 & -0.024 & 1.541 & -0.094 \\
\hline 2.60 & -0.040 & 0.563 & -0.035 & 1.506 & -0.097 \\
\hline 2.63 & -0.036 & 0.520 & -0.043 & 1.480 & -0.100 \\
\hline 2.65 & -0.034 & 0.478 & -0.040 & 1.453 & -0.102 \\
\hline 2.66 & -0.033 & -0.479 & 0.050 & -1.446 & -0.103 \\
\hline 2.69 & -0.029 & -0.438 & 0.057 & -1.419 & -0.106 \\
\hline 2.72 & -0.026 & -0.400 & 0.058 & -1.392 & -0.109 \\
\hline 2.75 & -0.023 & -0.364 & 0.059 & -1.366 & -0.112 \\
\hline 2.78 & -0.021 & -0.331 & 0.060 & -1.339 & -0.115 \\
\hline 2.81 & -0.018 & -0.299 & 0.059 & -1.306 & -0.117 \\
\hline 2.84 & -0.016 & -0.271 & 0.057 & -1.270 & -0.118 \\
\hline 2.87 & -0.014 & -0.244 & 0.055 & -1.244 & -0.118 \\
\hline
\end{tabular}




\begin{tabular}{|c|c|c|c|c|c|}
\hline 2.90 & -0.012 & -0.219 & 0.053 & -1.217 & -0.118 \\
\hline 2.93 & -0.010 & -0.197 & 0.050 & -1.189 & -0.117 \\
\hline 2.96 & -0.008 & -0.176 & 0.046 & -1.158 & -0.114 \\
\hline 2.99 & -0.006 & -0.156 & 0.042 & -1.121 & -0.111 \\
\hline 3.02 & -0.004 & -0.136 & 0.037 & -1.083 & -0.106 \\
\hline 3.05 & -0.001 & -0.115 & 0.033 & -1.045 & -0.099 \\
\hline 3.08 & 0.003 & -0.090 & 0.028 & -1.009 & -0.091 \\
\hline 3.11 & 0.007 & -0.064 & 0.023 & -0.971 & -0.082 \\
\hline 3.14 & 0.010 & -0.043 & 0.018 & -0.941 & -0.071 \\
\hline 3.17 & 0.012 & -0.027 & 0.012 & -0.913 & -0.059 \\
\hline 3.20 & 0.013 & -0.016 & 0.007 & -0.885 & -0.045 \\
\hline 3.23 & 0.014 & -0.009 & 0.001 & -0.856 & -0.029 \\
\hline 3.26 & 0.014 & -0.004 & -0.005 & -0.825 & -0.011 \\
\hline 3.29 & 0.014 & -0.001 & -0.011 & -0.795 & 0.010 \\
\hline 3.32 & 0.014 & 0.002 & -0.019 & -0.772 & 0.032 \\
\hline 3.35 & 0.014 & 0.003 & -0.026 & -0.746 & 0.058 \\
\hline 3.38 & 0.013 & 0.004 & -0.033 & -0.720 & 0.084 \\
\hline 3.41 & 0.013 & 0.004 & -0.039 & -0.694 & 0.113 \\
\hline 3.44 & 0.013 & 0.005 & -0.045 & -0.670 & 0.146 \\
\hline 3.47 & 0.012 & 0.005 & -0.052 & -0.645 & 0.182 \\
\hline 3.50 & 0.012 & 0.005 & -0.057 & -0.622 & 0.220 \\
\hline 3.53 & 0.011 & 0.005 & -0.064 & -0.599 & 0.261 \\
\hline 3.56 & 0.011 & 0.005 & -0.072 & -0.576 & 0.306 \\
\hline 3.59 & 0.011 & 0.005 & -0.079 & -0.555 & 1.036 \\
\hline 3.62 & 0.010 & 0.004 & -0.086 & -0.533 & 0.977 \\
\hline 3.64 & 0.010 & 0.004 & -0.089 & -0.522 & 0.019 \\
\hline 3.65 & 0.010 & 0.004 & -0.093 & -0.513 & 0.084 \\
\hline 3.68 & 0.010 & 0.004 & -0.101 & -0.492 & 0.237 \\
\hline 3.71 & 0.009 & 0.004 & -0.110 & -0.472 & 0.343 \\
\hline 3.74 & 0.009 & 0.003 & -0.119 & -0.453 & 0.423 \\
\hline 3.77 & 0.009 & 0.003 & -0.127 & -0.434 & 0.484 \\
\hline 3.80 & 0.008 & 0.003 & -0.135 & -0.415 & 0.535 \\
\hline 3.83 & 0.008 & 0.003 & -0.157 & -0.397 & 0.958 \\
\hline 3.86 & 0.008 & 0.003 & -0.304 & -0.379 & 0.837 \\
\hline 3.88 & 0.008 & 0.003 & -0.304 & -0.368 & -0.132 \\
\hline 3.89 & 0.007 & 0.003 & 0.383 & -0.361 & 0.111 \\
\hline 3.92 & 0.007 & 0.002 & 0.385 & -0.344 & 0.530 \\
\hline 3.93 & 0.007 & 0.002 & 0.376 & -0.344 & 0.898 \\
\hline 3.94 & 0.007 & 0.002 & 0.357 & -0.340 & -2.238 \\
\hline 3.95 & 0.007 & 0.002 & 0.350 & -0.327 & -2.177 \\
\hline 3.98 & 0.006 & 0.002 & 0.327 & -0.310 & -2.093 \\
\hline 4.01 & 0.006 & 0.002 & 0.310 & -0.293 & -1.982 \\
\hline 4.05 & 0.006 & 0.002 & 0.280 & -0.270 & -1.832 \\
\hline 4.08 & 0.006 & 0.002 & 0.262 & -0.256 & -1.720 \\
\hline 4.10 & 0.006 & 0.002 & 0.258 & -0.245 & -1.647 \\
\hline 4.13 & 0.005 & 0.001 & 0.241 & -0.230 & -1.538 \\
\hline 4.16 & 0.005 & 0.001 & 0.225 & -0.215 & -1.432 \\
\hline 4.19 & 0.005 & 0.001 & 0.211 & -0.200 & -1.329 \\
\hline 4.22 & 0.005 & 0.001 & 0.197 & -0.186 & -1.218 \\
\hline 4.25 & 0.004 & 0.001 & 0.184 & 0.172 & 1.135 \\
\hline 4.28 & 0.004 & 0.001 & 0.173 & 0.159 & 1.044 \\
\hline 4.31 & 0.004 & 0.001 & 0.161 & 0.147 & 0.958 \\
\hline
\end{tabular}




\begin{tabular}{|l|l|l|l|l|l|}
\hline 4.34 & 0.004 & 0.001 & 0.151 & 0.135 & 0.876 \\
\hline 4.37 & 0.004 & 0.001 & 0.141 & 0.124 & 0.800 \\
\hline 4.40 & 0.004 & 0.001 & 0.132 & 0.113 & 0.729 \\
\hline 4.43 & 0.003 & 0.001 & 0.124 & 0.104 & 0.664 \\
\hline 4.46 & 0.003 & 0.001 & 0.116 & 0.094 & 0.603 \\
\hline 4.49 & 0.003 & 0.001 & 0.108 & 0.086 & 0.548 \\
\hline 4.52 & 0.003 & 0.001 & 0.101 & 0.078 & 0.497 \\
\hline 4.55 & 0.003 & 0.000 & 0.095 & 0.071 & 0.450 \\
\hline
\end{tabular}




\section{Vibrational levels of some shallow states:}

Table (SM.3): The eigenvalues $\mathrm{E}_{v}$, the rotational constants $\mathrm{B}_{v}$, the centrifugal distortion constants $\mathrm{D}_{v}$, the abscissas of the turning points $\mathrm{R}_{\min }$ and $\mathrm{R}_{\max }$ and some calculated wavelengths of possible vibronic transitions of different shallow states of the $\mathrm{ZnF}$ molecule.

\begin{tabular}{|c|c|c|c|c|c|c|c|}
\hline state & $v$ & $\begin{array}{c}R_{\min } \\
(\AA)\end{array}$ & $\begin{array}{c}R_{\max } \\
(\AA)\end{array}$ & $\begin{array}{c}E_{v} \\
\left(\mathrm{~cm}^{-1}\right)\end{array}$ & $\begin{array}{c}B_{v} \times 10^{2} \\
\left(\mathrm{~cm}^{-1}\right)\end{array}$ & $\begin{array}{c}D_{v} \times 10^{6} \\
\left(\mathrm{~cm}^{-1}\right)\end{array}$ & $\begin{array}{c}\lambda_{v^{\prime} \leftrightarrow v^{\prime \prime}} \\
(\mu \mathrm{m})\end{array}$ \\
\hline \multirow{3}{*}{$(1)^{2} \Pi$} & 0 & 3.607 & 4.116 & 17.96 & 7.668 & 1.465 & \multirow{3}{*}{$\begin{array}{l}\lambda_{1 \leftrightarrow 0}=292.6 \\
\lambda_{2 \leftrightarrow 1}=314.8 \\
\lambda_{2 \leftrightarrow 0}=151.6\end{array}$} \\
\hline & 1 & 3.476 & 4.380 & 52.14 & 7.375 & 1.549 & \\
\hline & 2 & 3.399 & 4.604 & 83.91 & 7.062 & 1.685 & \\
\hline \multirow{6}{*}{$(1)^{4} \Sigma^{+}$} & 0 & 2.611 & 2.936 & 41.92 & 14.827 & 1.922 & \multirow{6}{*}{$\begin{array}{l}\lambda_{1 \leftrightarrow 0}=124.3 \\
\lambda_{2 \leftrightarrow 1}=134.2 \\
\lambda_{2 \leftrightarrow 0}=64.5\end{array}$} \\
\hline & 1 & 2.522 & 3.116 & 122.37 & 14.324 & 2.124 & \\
\hline & 2 & 2.472 & 3.254 & 196.90 & 13.836 & 1.894 & \\
\hline & 3 & 2.434 & 3.380 & 268.67 & 13.367 & 2.109 & \\
\hline & 4 & 2.405 & 3.500 & 336.43 & 12.926 & 2.033 & \\
\hline & 5 & 2.381 & 3.619 & 400.76 & 12.445 & 2.039 & \\
\hline \multirow{4}{*}{$(1)^{4} \Delta$} & 0 & 2.777 & 3.168 & 30.08 & 12.934 & 2.306 & \multirow{4}{*}{$\begin{array}{l}\lambda_{1 \leftrightarrow 0}=167.1 \\
\lambda_{2 \leftrightarrow 1}=172.6 \\
\lambda_{2 \leftrightarrow 0}=84.9\end{array}$} \\
\hline & 1 & 2.683 & 3.360 & 89.92 & 12.493 & 2.132 & \\
\hline & 2 & 2.624 & 3.517 & 147.85 & 12.025 & 2.232 & \\
\hline & 3 & 2.582 & 3.663 & 202.71 & 11.589 & 2.407 & \\
\hline \multirow{2}{*}{$(1)^{4} \Sigma^{-}$} & 0 & 2.950 & 3.368 & 25.51 & 11.478 & 2.377 & \multirow{2}{*}{$\lambda_{1 \leftrightarrow 0}=202.2$} \\
\hline & 1 & 2.824 & 3.581 & 74.98 & 11.127 & 2.530 & \\
\hline \multirow{11}{*}{$(3)^{4} \Pi$} & 0 & 2.561 & 2.848 & 56.11 & 15.646 & 1.230 & \multirow{11}{*}{$\begin{array}{l}\lambda_{1 \leftrightarrow 0}=91.0 \\
\lambda_{2 \leftrightarrow 1}=94.4 \\
\lambda_{2 \leftrightarrow 0}=46.3\end{array}$} \\
\hline & 1 & 2.482 & 2.984 & 166.01 & 15.287 & 1.244 & \\
\hline & 2 & 2.433 & 3.092 & 271.92 & 14.916 & 1.254 & \\
\hline & 3 & 2.397 & 3.188 & 374.05 & 14.573 & 1.245 & \\
\hline & 4 & 2.369 & 3.279 & 472.76 & 14.218 & 1.265 & \\
\hline & 5 & 2.345 & 3.366 & 567.92 & 13.875 & 1.283 & \\
\hline & 6 & 2.324 & 3.452 & 659.56 & 13.528 & 1.272 & \\
\hline & 7 & 2.306 & 3.537 & 747.87 & 13.189 & 1.315 & \\
\hline & 8 & 2.291 & 3.622 & 832.77 & 12.850 & 1.323 & \\
\hline & 9 & 2.277 & 3.708 & 914.26 & 12.497 & 1.357 & \\
\hline & 10 & 2.264 & 3.795 & 992.31 & 12.156 & 1.375 & \\
\hline \multirow{4}{*}{$(4)^{4} \Pi$} & 0 & 2.392 & 2.639 & 74.92 & 18.074 & 1.104 & \multirow{4}{*}{$\begin{array}{l}\lambda_{1 \leftrightarrow 0}=69.9 \\
\lambda_{2 \leftrightarrow 1}=76.2 \\
\lambda_{2 \leftrightarrow 0}=36.5\end{array}$} \\
\hline & 1 & 2.324 & 2.767 & 217.99 & 17.532 & 1.266 & \\
\hline & 2 & 2.286 & 2.870 & 349.19 & 16.911 & 1.408 & \\
\hline & 3 & 2.260 & 2.990 & 468.90 & 16.198 & 1.830 & \\
\hline \multirow{8}{*}{$(5)^{4} \Pi$} & 0 & 2.182 & 2.393 & 103.56 & 21.849 & 1.005 & \multirow{8}{*}{$\begin{array}{l}\lambda_{1 \leftrightarrow 0}=49.9 \\
\lambda_{2 \leftrightarrow 1}=52.3 \\
\lambda_{2 \leftrightarrow 0}=25.5\end{array}$} \\
\hline & 1 & 2.124 & 2.497 & 303.91 & 21.340 & 1.052 & \\
\hline & 2 & 2.089 & 2.580 & 494.99 & 20.821 & 1.106 & \\
\hline & 3 & 2.063 & 2.656 & 676.79 & 20.291 & 1.162 & \\
\hline & 4 & 2.043 & 2.730 & 849.42 & 19.754 & 1.219 & \\
\hline & 5 & 2.026 & 2.802 & 1013.06 & 19.208 & 1.283 & \\
\hline & 6 & 2.012 & 2.874 & 1167.86 & 18.657 & 1.338 & \\
\hline & 7 & 1.999 & 2.949 & 1314.12 & 18.101 & 1.430 & \\
\hline
\end{tabular}




\begin{tabular}{|c|c|c|c|c|c|c|}
\hline \multirow{2}{*}{8} & 1.988 & 3.025 & 1451.64 & 17.510 & 1.539 \\
\hline 9 & 1.979 & 3.104 & 1580.34 & 16.934 & 1.543 \\
\hline 10 & 1.971 & 3.185 & 1701.18 & 16.356 & 1.690 \\
\hline 11 & 1.963 & 3.269 & 1813.93 & 15.769 & 1.704 \\
\hline 12 & 1.956 & 3.357 & 1919.26 & 15.185 & 1.855 \\
\hline 13 & 1.951 & 3.449 & 2017.16 & 14.598 & 1.902 \\
\hline 14 & 1.945 & 3.545 & 2107.99 & 13.998 & 2.005 \\
\hline
\end{tabular}

